THERMAL TUNING OF PLANAR BRAGG GRATINGS IN SOI RIB WAVEGUIDES 


\title{
Thermal Tuning of Planar Bragg Gratings in Silicon-on-Insulator Rib Waveguides
}

\author{
by
}

SHABNAM HOMAMPOUR

B.Sc. (University of Toronto) 2007

A Thesis

Submitted to the School of Graduate Studies

in Partial Fulfilment of the Requirements for the Degree

Master of Applied Science (M.A.Sc.)

McMaster University

CC) Copyright by Shabnam Homampour, 2010. 
MASTER OF APPLIED SCIENCE (2010)

(Engineering Physics)

McMaster University

Hamilton, Ontario

TITLE: Thermal Tuning of Planar Bragg Gratings in Silicon-on-Insulator Rib Waveguides

AUTHOR: Shabnam Homampour

SUPERVISORS: Dr. P.E. Jessop, Dr. A.P. Knights

NUMBER OF PAGES: xi,142 


\section{Abstract}

Thermally tunable planar Bragg gratings in silicon-on-insulator (SOI) rib waveguides with external and integrated heaters were simulated, fabricated, and measured. Planar Bragg gratings were fabricated using selective silicon self-implantation at a dose of $2 \times 10^{15}$ ions $/ \mathrm{cm}^{2}$ to amorphize and increase the refractive index by 0.3 due to the induced damage. External heating resulted in a positive shift of 0.080 $\mathrm{nm} /$ degree in a good agreement with the $0.082 \mathrm{~nm} /$ degree predicted by theory. This demonstrated the tunability of planar Bragg gratings in SOI rib waveguides and motivated the fabrication of integrated heaters on waveguides. The planar surface of implanted Bragg gratings makes it desirable for subsequent processing such as surface bonding and its tunability makes it applicable as an active device.

Simulations performed with the heat transfer module of COMSOL in conjunction with beam propagation method (BPM) simulations predicted that application of $200 \mathrm{~mA}$ current to $100 \mathrm{~nm}$ thick aluminum strips is sufficient to generate a $10 \mathrm{de}$ gree temperature increase in a waveguide and that a minimum thickness of $300 \mathrm{~nm}$ of oxide layer is required to prevent mode loss in a waveguide due to the presence of the metal layer on top. Due to temperature restrictions on implanted damage and high temperatures involved in growth of oxide, non-cured spin-on-glass was used as the insulating layer between the rib waveguide and metal strips. Although, the combination of spin-on-glass (SOG) and aluminum worked on the test samples, it was unsuccessful on the device samples, such that the integrated heaters failed and the deposited aluminum affected the propagation optical mode.

The use of Bragg gratings to couple a broad spectrum of surface illuminating 
light to a multimode SOI slab waveguide was also demonstrated. This has potential applications in enhancing the long wavelength performance of solar cells. The addition of Bragg gratings on top of slab waveguides promotes light coupling, trapping reflected light and also increasing the optical path length of the incident beam, which increases the efficiency of the solar cells. Bragg gratings with a pitch of 500 $\mathrm{nm}$ were fabricated on part of the surface of a multimode planar SOI waveguide and the coupling of light into waveguides with and without Bragg gratings were measured. The measurements and the simulations were in good agreement, confirming that broadband Bragg grating couplers are a good candidate for coupling in more light with higher efficiencies. 


\section{Acknowledgements}

I would like to thank my supervisors, Dr. Paul Jessop and Dr. Andy Knights for their invaluable guidance, advice and support that led to the success of this project.

I would like to extend a great thank you to team Jessop especially Edgar Huante for his endless help and support and answering all of my quick questions and Chris Brooks for his advice, ideas and help in fabrication processing.

I would also like to thank the research staff at the Centre for Emerging Device Technologies (CEDT) especially Doris Stevanovic, Zhilin Peng, and Graham Pearson for providing their knowledge and expertise in training and helping me in fabrication of my devices.

I would also like to thank the research staff at the Canadian Centre for Electron Microscopy (CCEM) for their help and training for working with Scanning Electron Microscope (SEM).

Finally, I would like to thank my parents and brother for their love and support as always. 


\section{Contents}

1 Introduction 1

1.1 Optical Integration and Silicon Photonics _. . . . . . . . . . . 1

1.2 Bragg Gratings and Silicon Photonics . . . . . . . . . . . . 4

2 Background Theory 9

2.1 The Propagation of Guided Light . . . . . . . . . . . . . . . . 9

2.1.1 Maxwell's Equations and Electromagnetic Wave Equation . 11

2.1.2 Wave Propagation in Slab Waveguides . . . . . . . . . . 14

2.1.3 SOI Rib Waveguides and the Effective Index Method . . . . 19

2.1.4 Single Mode Condition for Rib Waveguides . . . . . . . . 20

2.1 .5 Mode Properties . . . . . . . . . . . . . . . . . 22

2.1.6 Beam Propagation Method and Mode profile . . . . . . 23

2.2 Bragg Gratings . . . . . . . . . . . . . . . . . . . . . . . . 24

2.2.1 Fundamentals of Bragg Gratings . . . . . . . . . . . 26

2.2.2 Contradirectional Coupled Mode Theory in a Periodic Structure ................... . 28

2.2.3 Coupling Coefficient for Index Modulated Gratings . . . . . 36

2.2.4 Coupling Coefficient for Relief Gratings . . . . . . . . . . . 39

2.2.5 Broadband Bragg Grating Couplers on SOI . . . . . . . . 40

2.3 Ion Implantation in Silicon . . . . . . . . . . . . . . . . . . 45

2.3.1 Fundamentals of Ion Implantation . . . . . . . . . . . . . 46

2.3.2 Amorphization of Silicon and Damage Profile . . . . . . . 48

2.3.3 Damage-Induced Refractive Index Modification and Loss . 50

2.4 Modeling Implanted Bragg Gratings in Waveguides . . . . . . . . . 53

2.4.1 Gratings with Loss . . . . . . . . . . . . . . . . . . 54

2.4.2 Propagation Losses due to Implantation . . . . . . . . . 57

2.5 Thermal Tuning of The Resonant Wavelength of Bragg Gratings . . 58

$2.5 .1 \quad$ External Heater . . . . . . . . . . . . . . . . . . . . . 59

2.5 .2 Integrated Heater . . . . . . . . . . . . . . . . . . 60 
3 Fabrication $\quad 64$

3.1 Fabrication of Silicon Photonic Devices . . . . . . . . . . . . 64

3.2 Fabrication of Bragg Gratings . . . . . . . . . . . . . . 66

3.2.1 Lithographic Based Fabrication Process . . . . . . . . . . . 67

3.2.2 Other Techniques for the Fabrication of Bragg Gratings . . . 71

3.3 Implanted Bragg Gratings in SOI Waveguides . . . . . . . . . . . . 72

3.4 Etched Gratings in SOI Waveguides . . . . . . . . . . . . . . . . 81

3.5 Integrated Heaters on Implanted Bragg Gratings in SOI Waveguides 82

3.6 Fabrication of Broadband Grating Coupler on SOI . . . . . . . . . . 84

3.7 Development and Application of Fabrication Processes . . . . . . . 85

3.7 .1 Wet Etch . . . . . . . . . . . . . . . 85

3.7 .2 Dry Etch . . . . . . . . . . . . . . . . 86

3.7.2.1 Chemical Etching . . . . . . . . . . . . 87

3.7.2.2 Physical Etching . . . . . . . . . . . . . . . 87

3.7.2.3 Ion-Enhanced Etching . . . . . . . . . . . . . . 88

3.7.2.4 Different Dry Etch Systems . . . . . . . . . . . 89

3.7.3 The Important Parameters in an Etch Process . . . . . . . . 94

3.7.3.1 Bosch Process . . . . . . . . . . . . . . . . . . . 94

3.7.3.2 The Effect of the Parameters . . . . . . . . 96

4 Experimental Results 99

4.1 Dry Etch Characterization . . . . . . . . . . . . . . . . . . . . . . 99

4.1.1 Analysis of Results . . . . . . . . . . . . . . . . . . . . . . 101

4.1.1.1 Effects of Power on Etch Rate and sidewall Angle 101

4.1.1.2 Effects of Passivation Cycle Flow Rate on Etch

Rate and Sidewall Angle . . . . . . . . . . . . . . . . . . . 104

4.1.1.3 Sidewall Roughness . . . . . . . . . . . . . . . 106

4.1.1.4 Uniformity . . . . . . . . . . . . . . . . . . 107

4.1 .2 Propagation Losses . . . . . . . . . . . . . . . . . . . . . 108

4.2 Thermal Tuning of the Resonant Wavelength of the Ion Implanted

Planar Bragg Gratings . . . . . . . . . . . . . . . . . . . . . . . . . 108

4.2.1 External Heater . . . . . . . . . . . . . . . . . . . . . . . . 108

4.2.1.1 Experimental Setup for Optical Characterization . 108

4.2.1.2 Transmission Spectra . . . . . . . . . . . . . 110

4.2.1.3 Loss Measurements . . . . . . . . . . . . . . . . 112

4.2 .2 Integrated Heater . . . . . . . . . . . . . . . . . . . . . . . 114

4.2.2.1 Experimental Setup for Optical Characterization . 114

4.2.2.2 Transmission Spectra . . . . . . . . . . . . . . . 114

4.2.2.3 Comparison to the Etched Gratings . . . . . . . . 117

4.3 Broadband Grating Coupler on SOI . . . . . . . . . . . . . . . . . 122 
5 Conclusion $\quad 126$

$\begin{array}{lr}\text { A Conferences and Publications } & 129\end{array}$

A How to Operate the Etcher STS 320PC 130 


\section{List of Figures}

1.1 A surface relief (Etched) grating on the right, An index modulated grating on the left ................. 5

2.1 A diagram of asymmetric slab waveguide . . . . . . . . . . . . . . 10

2.2 The first few computed modes in a planar waveguide structure . . . 18

2.3 A typical rib waveguide structure in SOI . . . . . . . . . . . . . . . 19

2.4 Effective Index Method for a rib waveguide . . . . . . . . . . . . . 20

2.5 Cross section of a rib waveguide . . . . . . . . . . . . . . 21

2.6 Passive grating components for optical circuits . . . . . . . . . . . . 25

2.7 Ion implanted Bragg gratings with period $\Lambda$. The forward and backward traveling modes can be coupled under Bragg condition . . . . 27

2.8 Expression of a perturbed wave in CMT. Such expression with an additional layer in the unperturbed section is called Additional Layer Method with a z-averaged index of refraction . . . . . . . . . . . . 29

2.9 A corrugated section of the dielectric waveguide with the incident and reflected intensities inside this section [1] . . . . . . . . . . 35

2.10 The contour map of the power propagation of TE polarized beam in gratings . . . . . . . . . . . . . . . . . . . . . . . 44

2.11 The plot of the power percentage of the TE mode vs. the distance under grating . . . . . . . . . . . . . . . . . . . . . . . . 44

2.12 TE Mode profile and the corresponding contour map of the transverse field for $200 \mathrm{~nm}$ oxide layer on left and that of $300 \mathrm{~nm}$ oxide layer on the right . . . . . . . . . . . . . . . . . 62

2.13 The heat transfer profile on top and the temperature change over time in the entire waveguide at the bottom left and the temperature difference between the top and bottom parts of the rib at the end at the bottom right . . . . . . . . . . . . . . . .

3.1 Standing waves produced by interference holography on a photosensitive material . . . . . . . . . . . . . . . . . . . . . 67

3.2 Lloyd's mirror setup . . . . . . . . . . . . . . . . . . . . . . . 68 
3.3 Fabrication process of implanted Bragg gratings on SOI waveguide. 73

3.4 Etched PR grating and the effect of the chromium grating windows. The length bar indicates $1 \mu \mathrm{m}$. The patterns on the surface are due to the gold coating for better SEM resolution. . . . . . . . . . . . 78

3.5 A completed ion implanted rib waveguide with a planar surface.

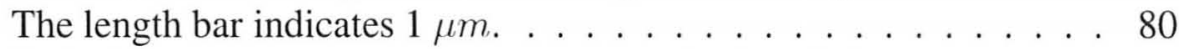

3.6 Etched grating on SOI rib waveguide. The length bar represents $100 \mathrm{~nm} . \ldots \ldots \ldots 1$

3.7 Fabrication process for integrating heaters . . . . . . . . . . . 82

3.8 SEM image of SOG on the rib. The length bar indicates $100 \mathrm{~nm}$. . . 83

3.9 Barrel Etcher system [2] . . . . . . . . . . . . . . . . . . . . . . . 93

3.10 ICP Dry Etch system [3] . . . . . . . . . . . . . . . . . . . . . . . 94

4.1 Graph of Etch Rate vs. Power . . . . . . . . . . . . . . . . . . . . 101

4.2 Graph of Sidewall Angle vs. Power . . . . . . . . . . . . . . . . . 102

4.3 Graph of Etch Rate vs. $\mathrm{C}_{4} \mathrm{~F}_{8}$ Flow Rate. . . . . . . . . . . . . . . . 103

4.4 Graph of Sidewall Angle vs. $\mathrm{C}_{4} \mathrm{~F}_{8}$ Flow Rate. . . . . . . . . . . . 104

4.5 A positively tapered sidewall on left with $60 \mathrm{~W}$ power and A Sidewall with Undercut is illustrated on right with power set at $100 \mathrm{~W}$. The $\mathrm{C}_{4} \mathrm{~F}_{8}$ and $\mathrm{SF}_{6}$ flow rates were kept at 32 and $16 \mathrm{sccm}$ respectively. The white bar indicates $200 \mathrm{~nm}$. . . . . . . . . . . . . . . . 105

4.6 Vertical Sidewall with $75 \mathrm{~W}$ power. The flow rates were same as that in above figures. The white bar indicates $200 \mathrm{~nm}$. . . . . . . . . 105

4.7 Experimental setup for optical characterization of samples with external heater. . . . . . . . . . . . . . . . . . . . . . . . . . . . . 109

4.8 The typical transmission spectrum of rib waveguides with implanted Bragg gratings . . . . . . . . . . . . . . . . . . . . 110

4.9 Transmission spectrum of planar Bragg grating rib waveguide for different temperatures. . . . . . . . . . . . . . . . . . . . . . . . . 112

4.10 Measured and modeled shift in the resonant wavelength of the grating using $d \lambda / d T=((1 / n)(d n / d T+\alpha) \lambda \ldots \ldots . . \ldots . . . . .113$

4.11 The transmission spectrum of an ion implanted sample with integrated aluminum heaters. A small dip close to the expected wavelength is observed. . . . . . . . . . . . . . . . . . . . 115

4.12 A transmission spectrum obtained from the ion implanted samples with integrated heater showing no bragg dip. . . . . . . . . . . 116

4.13 SEM image of an ion implanted rib with aluminum pattern and SOG. The white bar indicates $1 \mu \mathrm{m}$ length. . . . . . . . . . . . . 116

4.14 A TE mode transmission power spectrum of an etched grating. . . . 117

4.15 SEM image of an etched grating sample with aluminum pattern and SOG. The white bar indicates $1 \mu m$ length. . . . . . . . . . . . 118 
4.16 Contour map of the field when aluminum strip is deposited $0.5 \mu \mathrm{m}$ from a rib waveguide . . . . . . . . . . . . . . . . . . . . . . . . . . . . . . . . . . . . . . . . .

4.17 The heat transfer profile . . . . . . . . . . . . . . . . . . . . 119

4.18 The plot on left shows the temperature variance across the width of the rib at $y=2.3 \mu \mathrm{m}$ and the variation of temperature from bottom to top of the rib at $x=0$ is shown on the one on right. . . . . . . . 120

4.19 The heat transfer profile. . . . . . . . . . . . . . . . . . 121

4.20 The plot on left shows the temperature variance across the width of the rib and the variation of temperature from bottom to top of the rib is shown on the one on right. . . . . . . . . . . . . . . . 121

4.21 The contour map of the field when aluminum strips are deposited on each side of the rib at a $0.5 \mu \mathrm{m}$ from the rib waveguide. . . . . . 122

4.22 Experimental setup for optical characterization of the broadband grating coupler. . . . . . . . . . . . . . . . . . . . . 123

4.23 A typical plot obtained in the measurements of the broadband grating coupler with a fitted gaussian . . . . . . . . . . . . . . . . 123

4.24 The summary of the measurements is shown in top and the theory in the bottom plot. The distance is the amount traveled from the border between the grating and unetched region. . . . . . . . . . . 125 


\section{Chapter 1}

\section{Introduction}

\subsection{Optical Integration and Silicon Photonics}

As industry progresses according to Moore's law there is a constant drive to reduce device size and improve performance. As this migration proceeds more limitations must be conquered, for example limitations in size reduction due to thermal isolation and heat dissipation in the chips or the bandwidth and distance requirements in telecommunication. On-chip metal interconnects represent a major obstacle to further increases in device density. Therefore, replacement of electrical wires with optical beams and even substitution of separate optical devices with integrated optical devices and integration of optics and electronics on one chip is essential for the further development of the technology.

Integrated optics and replacement of wires and electrical circuits with optical beams for signal processing has been a major research topic since the early 1960's. As optical fibers replace copper cables, it becomes necessary for many of the electronic components to be replaced by their equivalent optical components such as 
Sh. Homampour - Masters Thesis

filters, switches, and splitters, and it is highly desirable that they be fabricated in a planar surface. The substitution of optical integrated circuits for electrical integrated circuits improves the switching times, batch fabrication economy, and low loss of the system. Not only does the optical integrated circuit have smaller size, weight and lower power consumption but also expanded frequency division multiplexing and increased bandwidth [4]. Although the concept of integrated optics has existed for long time, only recently have the proper conditions for development of these technologies emerged.

Employing silicon as the material of choice for the optical part of the devices has been an important topic in integrated optics since it is the optimal choice for the electrical circuits and utilizing silicon adds more functionality to the chips and makes the production of an active optical device possible. For instance, the variation in free carrier density that accompanies the basic functionality of transistors can be used to modulate the phase of the light. Thus, a new level of performance and functionality can be achieved along with reductions in power and size and cost [5].

The concept of silicon as the platform for integrated optics was substantially advanced by the work of Soref and Pogossian in early 1990s. These works presented the possibility of fabrication of micrometer-size single mode waveguides in silicon via standard photolithography $[6,7]$.

Silicon inherently is restricted as an optical material due to its indirect bandgap, which makes light emission inefficient, and it has centrosymmetric inversion symmetry, which precludes an electro-optic (Pockels) effect [8]. Optical waveguiding in silicon is conveniently possible using Silicon-on-Insulator (SOI) material where the silicon device layer is separated from the bulk silicon crystal with an oxide layer $\left(\mathrm{SiO}_{2}\right)$. The high contrast in the index of refraction of silicon and silicon 
dioxide provides the essential optical confinement for guided modes to propagate in the device layer [9]. This strong confinement between the layers in SOI not only enhances the miniaturizing of the features to the size required for economic compatibility with IC processing but also makes nonlinear optical interactions possible on the chip scale. Moreover, SOI is an ideal platform for creating planar optical confinement and integrating photonics and microelectronic devices. Therefore, functions like optical amplification and wavelength switching, that are central functions of multiwavelength communication and signal processing that were considered beyond the reach of silicon, are now developed $[10,11]$.

The absence of Pockels effect in silicon precludes the traditional method of optical modulators, but can be overcome by modulation of the free-carrier density. This makes possible direct changes in the optical intensity by free carrier absorption, or changing the index of refraction of the material that would cause changes in intensity when incorporated into an interferometer $[8,10]$.

The non-idealities of silicon structures, such as surface scattering, coupling loss between fiber mode and silicon rib waveguide modes ${ }^{1}$, and low light emission efficiency add to the disadvantages of using silicon as the light propagating material. However, such disadvantages can be overcome to some extent by reducing the size of the waveguides and using tapered fibers for fiber-waveguide coupling.

Moreover, the low cost, abundance and purity of the crystalline silicon in addition to its compatibility with CMOS fabrication processes ${ }^{2}$ and established facilities makes it a good candidate for optical devices and photonics. Silicon has a high thermal conductivity, large thermo-optic coefficient, and high optical damage threshold.

\footnotetext{
${ }^{1}$ due to their high modal mismatch

${ }^{2}$ This process accounts for more than $\% 95$ of the semiconductor chips [12]
} 
Sh. Homampour - Masters Thesis

It is transparent to wavelengths greater than $1.1 \mu \mathrm{m}$ which makes it a great choice for functionality of optical devices in the standard telecom window between $1.3 \mu \mathrm{m}$ to $1.55 \mu \mathrm{m}$. Materials like $\mathrm{LiNbO}_{3}, \mathrm{GaAs}$, and $\mathrm{InP}[5,13]$ have been used for fabrication of devices such as modulators and filters due to their excellent electrooptic properties, but demands for greater bandwidth, lower power and monolithic integration of optics and microelectronics on one substrate make silicon a better candidate for enhancing photonics. In the last few years silicon photonics has progressed towards active devices, including modulators and amplifiers. High speed electrooptic modulators with speed of up to $10 \mathrm{~Gb} / \mathrm{s}$ [14] have been demonstrated.

Silicon photonics has diverse applications from biosensors and lab-on-a-chip devices to detectors, switches, couplers, and filters in telecommunications. One of the possible methods for constructing integrated optical filters is to use Bragg gratings. These fine periodic structures have a period comparable to the optical wavelength and are widely used as modulators, bandpass filters, and couplers with the advantage of having suitable dimensions for integration.

\subsection{Bragg Gratings and Silicon Photonics}

Bragg gratings are implemented either as a Fiber Bragg Gratings (FBG) or Integrated Bragg Gratings. With the discovery of the photosensitivity in optical fibers, Fiber Bragg Gratings as an in-fiber optical component have been developed. Not only can they perform the primary functions like reflection and filtering with high efficiency and low loss [15] but these devices are also fundamental to optical networks with great impact on telecommunication and optical fiber sensing. FBG dispersion compensators [16], biosensors [17], and optical storage are some of the 
exciting applications of Fiber Bragg Gratings.

Integrated Bragg gratings can be constructed by physical corrugation of the substrate instead of relying on the photorefractive effect. This versatility in fabrication applies more functionality to such gratings. For instance, fabrication of Bragg gratings in $\mathrm{Si}$ and $\mathrm{InP}$ has become possible. Moreover, the combination of multiple levels of lithography, which leads to more precise alignment and better integration, better control over dimensions, and closer packed devices, is another advantage of such implementation.

Integrated Bragg Gratings can be categorized into two types: Surface relief gratings are defined by physically removing parts of the waveguide layer; index modulated gratings are defined when an index modulation is induced in the layer. The two types of gratings are illustrated in Figure 1.1.
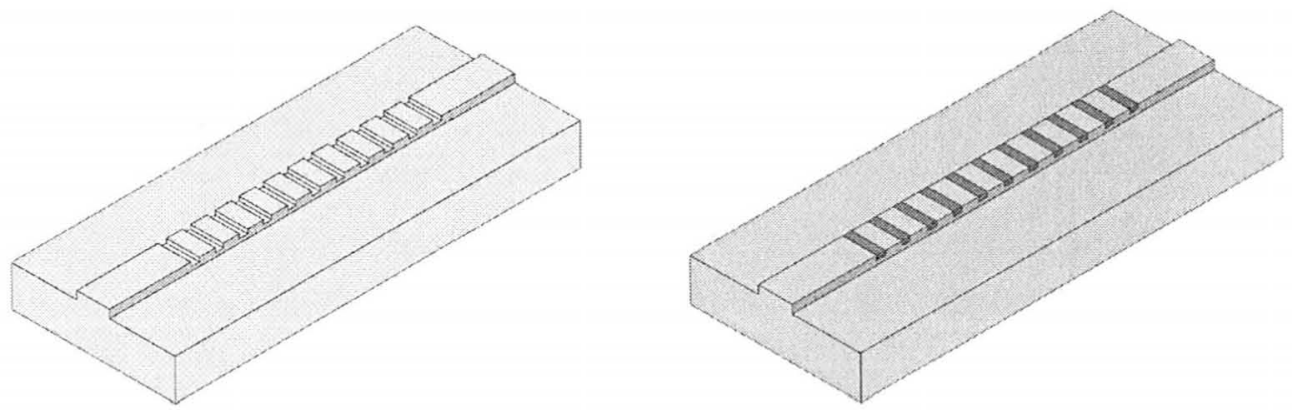

Figure 1.1: A surface relief (Etched) grating on the right, An index modulated grating on the left

Surface relief gratings are usually passive devices with large coupling coefficient. These devices have many sensing applications such as temperature sensors [18] and biosensors [19] as well as others like an antireflective structure [20].

Index modulated gratings can be formed not only through photorefractive ef- 
fects but also doping, the electro-optic effect ${ }^{3}$, and the acousto-optic effect ${ }^{4}$ [21]. In these devices the planar surface of the waveguide is retained and active device capabilities are possible. The preservation of the planarity is an important attribute as surface sensitive bonding techniques find ever-greater use in integrated photonics. One of the primary applications of integrated Bragg gratings has been the Distributed Feedback Laser or DFB where diffraction gratings provide the optical feedback that controls laser action. These semiconductor lasers were originally fabricated using III-V materials but other materials including metal [22], have been used for the gratings. Bragg gratings have been fabricated on different platforms like Polymers [23], $\mathrm{LiNbO}_{3}$ [24], Silicon-On-Insulator (SOI) [25], and Silica [26].

In applications involving Bragg gratings on SOI, surface relief gratings are the more commonly used option. These gratings are usually patterned through lithography techniques and then etching. Realizing grating patterns using a holography system and RIE etcher [27], e-beam lithography, and focused ion beam milling [28] are some of the common procedures. These devices are inherently passive but are electrically and thermally tunable. However, their non-planar surface limits their applications as technology progresses towards smaller, more compact, and three dimensional optical circuits where surface bonding and subsequent processing is required.

Therefore, index modulating gratings would be a better option. These are possible due to an important property of silicon explored by Baranova [29]. As ions are implanted in crystalline silicon, their imparted energy damages the lattice and as the damage accumulates the index of refraction of the silicon increases. With very

\footnotetext{
${ }^{3}$ by applying electric field

${ }^{4}$ by exciting a surface acoustic wave
} 
high implantation doses, the damaged crystalline silicon transforms to the amorphus state with the index of refraction 0.3 greater than that of the crystalline silicon [30]. Thus, by ion implanting the surface of the SOI substrate selectively and amorphizing the surface in a periodic pattern, an index modulation can be induced and planar Bragg gratings can be formed. However, a loss due to the produced damage, first explored by Fan and Ramdas [31], is introduced to the device.

One of the key issues for many integrated optical devices is having high wavelength selectivity, and the ability to tune the output wavelength is important to the operation of such devices. Moreover, the wavelength tuning capability is valuable for fine tuning when the target wavelength is not met during the fabrication. Thermal tuning of the resonant wavelength is one of the common possibilities and the large thermo-optic coefficient of silicon and its good thermal conductivity enhances silicon's tuning ability. Using this capability of the silicon is a simple and cost effective way to tune the devices at a moderate modulation speed [11]. Thus, thermal tuning of the planar Bragg gratings will introduce a new level of optical integration and efficiency.

The goal of this thesis is to explore the viability of thermal tuning of planar Bragg gratings in SOI rib waveguides with both external and integrated heaters and a comparison between the effects and efficiency of each technique.

Chapter 2 explores the background theory of propagation of guided light in SOI rib waveguides. It will cover the fundamentals of Bragg gratings, Contradirectional Coupled Mode Theory as the method used to predict the response of the ion implanted gratings to the incident light, the coupling coefficients and grating strength, and broadband grating couplers on SOI. The modeling of implanted Bragg gratings in waveguides will be discussed after fundamentals of ion implantation, amor- 
phization of silicon, and the damage-induced refractive index modifictaions are described. Finally, the thermal tuning of the resonant wavelength of Bragg gratings with both external and integrated heater is discussed.

Chapter 3 outlines the fabrication of silicon photonic devices, fabrication of Bragg gratings, and the implanted and etched Bragg gratings in SOI waveguides. Fabrication of integrated heaters on implanted Bragg gratings in SOI waveguides, broadband grating coupler on SOI, and the development and application of fabrication processes are also discussed.

Chapter 4 presents the results of the characterization of the RIE etcher (critical in the fabrication of present and future SOI devices at McMaster and up until now uncharacterized), results of the thermal tuning of the planar Bragg gratings, and results of the primary measurements towards a broadband grating coupler on SOI.

Chapter 5 offers some conclusions and suggestions for future work. 


\section{Chapter 2}

\section{Background Theory}

\subsection{The Propagation of Guided Light}

Light propagation and light and matter interactions have been studied for many centuries. The fundamental principles can be explained using Maxwell's equations by considering light as an electromagnetic wave. However, simpler models such as the Ray optical model can be employed to investigate some important phenomena. The principles governing light propagation and its interaction with media are covered in detail in $[1,4,5,21,32]$

Ray optics is based on Snell's law. Considering a light ray propagating in a medium with index of refraction $\mathrm{n}_{1}$ and impinging at an interface with angle $\theta_{1}$, the light will be partially reflected and partially transmitted to the second medium with index of refraction of $n_{2}$. The relationship between the indices of refraction and incident and refracted angles is given by Snell's law ${ }^{1}$.

\footnotetext{
${ }^{1}$ Snell's law can simply be shown experimentally and it is also a natural consequence of Maxwell's Electromagnetic Theory [33]
} 


$$
n_{1} \sin \left(\theta_{1}\right)=n_{2} \sin \left(\theta_{2}\right)
$$

Snell's law can be used to explain the confinement of propagating light in a dielectric layer with higher index of refraction surrounded by materials with lower index of refraction. A diagram of such layout is shown in Figure 2.1. This diagram can be considered as an asymmetric dielectric slab waveguide since the surrounding materials have different indices of refraction.

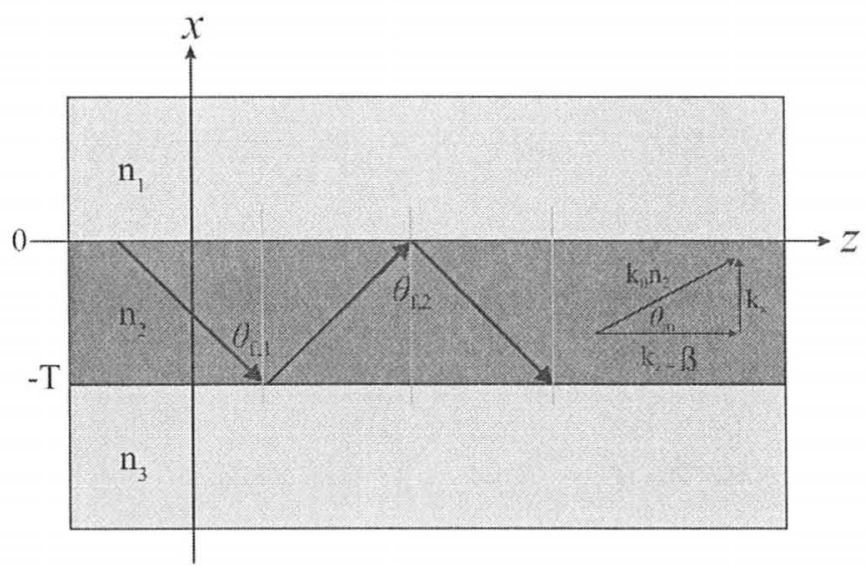

Figure 2.1: A diagram of asymmetric slab waveguide

In the above figure, $n_{2}>n_{3}>n_{1}$ with $\theta_{f, 1}$ and $\theta_{f, 2}$ being the angles of incidence at the interfaces. When the angle of incidence at the interfaces is greater than the critical angle at both the substrate and the cover interfaces, the light will be confined in the middle layer and will propagate in a zig-zag pattern. If the incident angle is less than either of the critical angles, there will be a large radiation loss at that interface. It can also be shown that due to phase matching considerations only discrete guided-modes, and corresponding angles of incidence, exist and their characteristics depend on the geometry of the waveguide. 
Using Maxwell's equations or wave optics to describe the light propagation is essential when a full description of the propagating modes is required, which is the case here. Thus, the modal characteristics of confined light in one and two dimensional waveguides will be examined using Maxwell's equations in the following sections.

\subsubsection{Maxwell's Equations and Electromagnetic Wave Equation}

By the ground work of James Clerk Maxwell the relationship between the electric field, magnetic field, and their sources is established. This set of equations in addition to the Lorentz force law completes the set of laws of classical electromagnetism and can explain all the interactions among electric, magnetic, electromagnetic, and optical phenomena. Moreover, these equations govern the behavior of light as an electromagnetic wave in media. Maxwell equations are expressed as

$$
\begin{array}{r}
\nabla \times \mathbf{E}=-\frac{\partial \mathbf{B}}{\partial t} \\
\nabla \times \mathbf{H}=\mathbf{J}+\frac{\partial \mathbf{D}}{\partial t} \\
\nabla \cdot \mathbf{D}=\rho \\
\nabla \cdot \mathbf{B}=0
\end{array}
$$

In the above equations $\mathbf{E}$ and $\mathbf{H}$ represent time varying electric and magnetic fields respectively, while $\mathbf{D}$ and $\mathbf{B}$ are electric and magnetic flux density. $\mathbf{J}$ is the current density and $\rho$ corresponds to the charge density. The current and charge 
density can be considered as the source of electromagnetic radiation and in the optical case in dielectrics are often considered to be zero ${ }^{2}$. The fields and flux densities are related through constitutive relations

$$
\begin{aligned}
\mathbf{D} & =\epsilon \mathbf{E} \\
\mathbf{B} & =\mu \mathbf{H}
\end{aligned}
$$

where $\epsilon$ is the permittivity of the medium and $\mu$ is the permeability of the medium. These two equations are employed to obtain a unique solution for electric and magnetic fields. It is also helpful to recall Ohm's law.

$$
\mathbf{J}=\sigma \mathbf{E}
$$

To derive the wave equation, one takes the curl of both sides of the equation 2.2a and substitutes for $\mathbf{B}$ field, $\nabla \times \mathbf{H}, \mathrm{J}$, and $\mathbf{D}$ respectively using equations $2.3 \mathrm{~b}$, $2.2 \mathrm{~b}, 2.4$, and $2.3 \mathrm{a}$, resulting in

$$
\nabla \times \nabla \times \mathbf{E}=-\mu\left[\sigma \frac{\partial \mathbf{E}}{\partial t}+\epsilon \frac{\partial^{2} \mathbf{E}}{\partial t^{2}}\right]
$$

applying the following vector identity

$$
\nabla \times \nabla \times \mathbf{E}=\nabla(\nabla \cdot \mathbf{E})-\nabla^{2} \mathbf{E}
$$

we arrive at the wave equation which describes the propagation of electromagnetic waves in a homogenous and isotropic medium with $\sigma=0$.

\footnotetext{
${ }^{2}$ which still yields to a non-zero solution meaning there exist electromagnetic field in the absence of any current or charge
} 
Sh. Homampour - Masters Thesis

$$
\nabla^{2} \mathbf{E}=\mu \epsilon \frac{\partial^{2} \mathbf{E}}{\partial t^{2}}
$$

The wave equation can be written for magnetic field similarly and it relates the space and time dependence of the field. Solutions to the wave equation mathematically describe modes of the form

$$
\mathbf{E}(\mathbf{r}, t)=E_{0}(\mathbf{r}, t) e^{i(\omega t-\mathbf{k} . \mathbf{r})}
$$

where $E_{0}(\mathbf{r}, \mathrm{t})$ is the slowly varying amplitude of the harmonic wave, $\mathrm{k}$ is the propagation constant, and $\omega$ is the angular frequency. In the situation where the amplitude $E_{0}(\mathbf{r}, \mathrm{t})$ is constant in time and space, the wave solution reduces to a plane wave. Substitution of such a solution into the wave equation results in

$$
k^{2} \mathbf{E}=\omega^{2} \mu \epsilon \mathbf{E}
$$

and simplifying this equation leads to the dispersion relation as follows

$$
k^{2}=\omega^{2} \mu \epsilon
$$

The phase velocity, $v$, is defined using the dispersion relation as

$$
v=\frac{\omega}{k}=\frac{1}{\sqrt{\mu \epsilon}}
$$

and combining equation 2.11 and the definition of index of refraction

$$
n=\frac{c}{v}
$$


The index of refraction can be written as

$$
n=c \sqrt{\mu \epsilon}=\frac{\sqrt{\mu \epsilon}}{\sqrt{\mu_{0} \epsilon_{0}}}
$$

Therefore, assuming $\mu=\mu_{0}{ }^{3}$ the index of refraction of a dielectric is expressed as

$$
n=\sqrt{\frac{\epsilon}{\epsilon_{0}}}=\sqrt{\epsilon_{r}}
$$

\subsubsection{Wave Propagation in Slab Waveguides}

Slab/Planar waveguides are characterized by the parallel planar boundaries confined in one direction (the $x$ direction in Figure 2.1 but infinite in extent in the lateral directions. Although they have limited practical applications in integrated optical circuits, their mathematical analysis is much simpler than that of practical waveguides with rectangular cross section. An example of such a slab waveguide is illustrated in Figure 2.1.

In this structure, a dielectric layer is surrounded by layers with lower index of refraction. Light can propagate within the dielectric layer in an in-plane direction as a result of total internal reflection. To characterize the propagating wave, the wave equation can be applied in each of the layers and solved by considering the continuity relations at each interface.

Assuming that light propagates as monochromatic plane waves in $z$ direction, the electromagnetic fields vary as

\footnotetext{
${ }^{3}$ since in dielectrics are not magnetic
} 


$$
\begin{aligned}
\mathbf{E}(\mathbf{r}, t) & =\mathbf{E}(x, y) e^{i(\omega t-\beta z)} \\
\mathbf{H}(\mathbf{r}, t) & =\mathbf{H}(x, y) e^{i(\omega t-\beta z)}
\end{aligned}
$$

where $\beta$ is the propagation constant in $z$ direction i.e. $\beta=\mathrm{k}_{z}$ the $z$ component of the wave vector. It is also defined as

$$
\beta=N k_{0}
$$

where $N$ is the effective index of refraction, which can be thought of as the refractive index of the mode as it propagates within the waveguide. We need only to consider the electric field here, since the results will be analogous for magnetic field. Therefore, the wave equation reduces to

$$
\frac{\partial^{2} \mathbf{E}(x, y)}{\partial x^{2}}+\frac{\partial^{2} \mathbf{E}(x, y)}{\partial y^{2}}+\left[k_{0}^{2} n^{2}-\beta^{2}\right] \mathbf{E}(x, y)=0
$$

where $n$ assumes the values $n_{1}, n_{2}$, or $n_{3}$, as appropriate in each layer. Since the waveguide is invariant in y direction, the above equation can be written for the three layers as

$$
\frac{\partial^{2} \mathbf{E}(x)}{\partial x^{2}}+\left[k_{0}^{2} n^{2}-\beta^{2}\right] \mathbf{E}(x)=0
$$

The solutions will be either sinusoidal or exponential functions of $x$, depending on $\beta$ and the sign of the second term in the above equation at constant frequency $\omega$. When the requirement for total internal reflection i.e. $k_{0} n_{3}<\beta<k_{0} n_{2}$ is met, then the solution for TE polarization can be written as 


$$
E_{y}=\left\{\begin{array}{ccl}
E_{1} e^{-\gamma_{1} x} & x>0 & \text { cover } \\
E_{2} \cos \left(k_{x} x+\varphi\right) & 0 \geq x \geq-T & \text { guiding layer } \\
E_{3} e^{\gamma_{3}(x+t)} & x<-T & \text { substrate }
\end{array}\right.
$$

where,

$$
\begin{aligned}
& \gamma_{1}^{2}=\beta^{2}-n_{1}^{2} k_{0}^{2} \\
& k_{x}^{2}=n_{2}^{2} k_{0}^{2}-\beta^{2} \\
& \gamma_{3}^{2}=\beta^{2}-n_{3}^{2} k_{0}^{2}
\end{aligned}
$$

This shows that the solution is sinusoidal in region II while it decays exponentially in the other two regions. Thus, the energy carried by these modes will be confined in the vicinity of guiding layer, but a portion of the energy is contained in the exponential tails in the substrate and cover. Considering the boundary conditions $^{4}$, the allowed values for $\beta$ are discrete and the number of confined modes depends on the indices of refraction of the layers, the frequency, and the thickness of the guiding layer. For fixed frequency and indices, the number of the guided modes increases proportional to the thickness of the layer.

Applying the boundary conditions to the equations 2.20 leads to the eigenvalue equation relating the parameters to the discrete modes. Considering TE modes, for which $E=\left(0, E_{y}, 0\right)$ and $H=\left(H_{x}, 0, H_{Z}\right), E(x)$ and $\partial E(x) / \partial x$ are continuous at $x=0$. This leads to

\footnotetext{
${ }^{4}$ i.e. $E(x)$ and $\partial E(x) / \partial x$ must be continuous at the interfaces
} 


$$
\begin{array}{r}
E_{1}=E_{2} \cos \varphi \\
\tan \varphi=\frac{\gamma_{1}}{k_{x}}
\end{array}
$$

Similarly, at $x=-T$

$$
\begin{array}{r}
E_{3}=E_{2} \cos \left(-k_{x} T+\varphi\right) \\
\tan \left(k_{x} T-\varphi\right)=\frac{\gamma_{3}}{k_{x}}
\end{array}
$$

Combining and rearranging $2.22 \mathrm{a}-2.23 \mathrm{~b}$ equations leads to the following eigenvalue equation

$$
\arctan \left(\frac{\gamma_{1}}{k_{x}}\right)+\arctan \left(\frac{\gamma_{3}}{k_{x}}\right)=k_{x} T+m \pi
$$

where $m=0,1, \ldots$ denotes the mode numbers. The $\mathrm{TE}_{0}$ is the fundamental mode of the waveguide with largest effective index and closest angle to $90^{\circ}$. Each allowed mode has a corresponding propagation constant $\beta_{m}$ that is given by

$$
\beta_{m}=k_{0} n_{2} \sin \left(\theta_{m}\right)
$$

where $\theta_{m}$ is the corresponding reflection angle. If $\beta>k_{0} n_{2}$ then the electric field will be exponential in all three layers and field will increase without bonding to the waveguide. Hence, the solution won't be physically realizable. Moreover, if $k_{0} n_{1}<\beta<k_{0} n_{3}$ or $0<\beta<k_{0} n_{1}$ then the solution is sinusoidal in regions II and III or in all three regions, which leads to the radiation modes of the waveguide. 
As mentioned before, the number of discrete modes depends on the indices of refraction and frequency as well as the thickness of the guiding layer. Therefore, there exists a cut-off in the number of modes allowed for a specific wavelength and device setup. The cut-off point corresponds to the point where the reflection angle becomes less than the critical angle for total internal reflection. In the case of asymmetric waveguide, where $n_{1}$ is very much less than $n_{3}$ which is very common in optical integrated circuits, the required relation between the indices for a given number of modes is given by equation 2.26 [34]

$$
\triangle n=n_{2}-n_{3}=(2 m+1)^{2} \lambda_{0}^{2} /\left(32 n_{2} t^{2}\right)
$$

The cut-off is very important since it allows tailoring of the waveguide parameters to allow only certain number of modes to propagate. The physical optics (electromagnetic wave) approach for solving the possible modes in a waveguide is more useful for optical integrated circuits since it allows obtaining the mode profile of the propagating mode. The first few TE modes are illustrated in the Figure 2.2

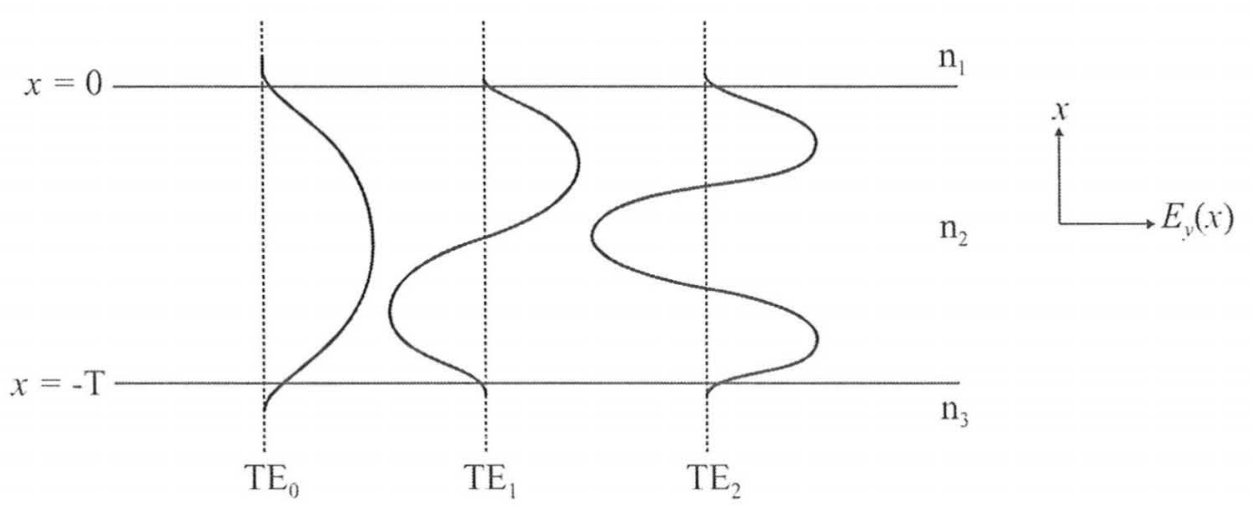

Figure 2.2: The first few computed modes in a planar waveguide structure 


\subsubsection{SOI Rib Waveguides and the Effective Index Method}

In order to have a practical waveguide, lateral confinement is necessary. Using rib structures is one of the common methods to do so, especially in SOI platforms. A typical rib waveguide is illustrated in Figure 2.3

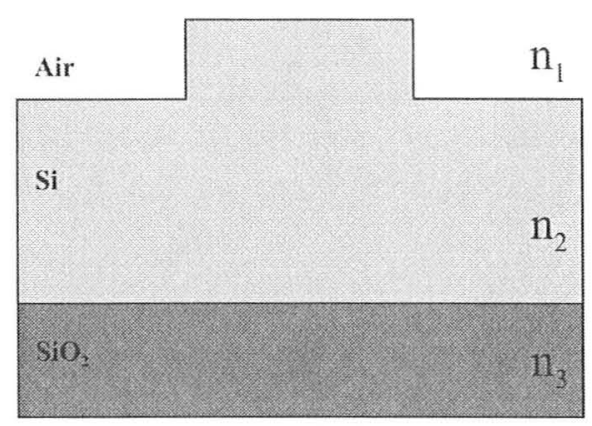

Figure 2.3: A typical rib waveguide structure in SOI

In the SOI waveguides considered in this thesis, the silicon guiding layer is a few micrometers thick and the thickness of the silicon dioxide layer is about half a micron. The buried oxide layer is to prevent the penetration of the field associated with the optical mode into the substrate. Thus, this layer should be thicker than the evanescent fields associated with the modes.

Unlike slab waveguides, rib waveguides do not have any analytical solutions hence, intensive numerical solutions are required to solve for the modes. The Effective Index Method is one of the common approximation methods employed to solve for the modes. In this approach the goal is to solve for the propagation constants by separating the two dimensional problem into two, one-dimensional planar waveguide problems; a horizontal one and a vertical one, (as shown in the Figure 2.4) and then solving the corresponding planar waveguide eigenvalue equations. The complication of this method is in choosing the correct polarization eigenvalue equation. 

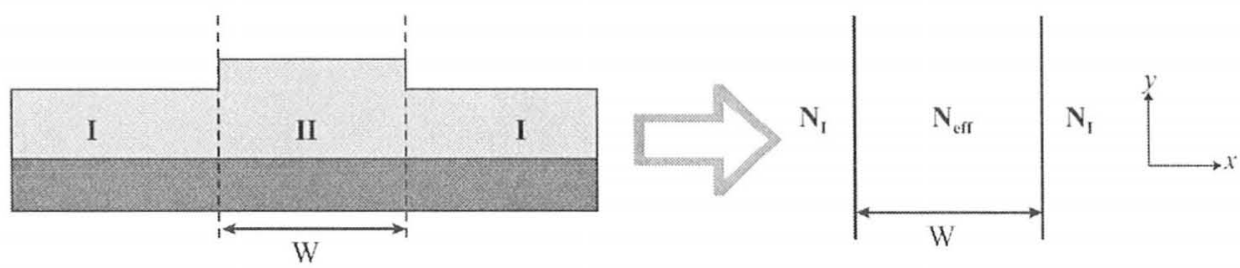

Figure 2.4: Effective Index Method for a rib waveguide

To clarify more, if the TE-polarized modes of the rib waveguide are to be solved for, then first regions I and II are each considered as a slab waveguides confined in the $x$ direction and the TE effective index of each is computed. Then, a slab waveguide confined in the $y$ direction (i.e. laterally) is solved for, based on the calculated effective index of each region. One solves for the TM-polarized modes of the lateral slab waveguide to obtain the effective index of the entire structure. To solve for the TM-polarized modes of the rib waveguide the polarizations are reversed. To confine the modes in the rib when slab waveguides in regions I and II are considered single mode must have $N_{I I}>N_{I}$.

\subsubsection{Single Mode Condition for Rib Waveguides}

It was not till 1991 that the possibility of having a single mode rib waveguide with a large cross section i.e. several microns high and several microns wide was realized by Soref et al.[6]. Prior to this work it was assumed that a rib waveguide in silicon must have a very small cross section, with a thickness similar to that of the single mode slab waveguide, to support propagation only in the lowest order mode. This would lead to a great amount of coupling loss due to the dimensional mismatch between the waveguide and optical fiber cross sections.

It was shown that a waveguide that is multimode in vertical direction, can still function as a single mode if the ratio of its height to width is appropriate. In this 
case the propagation constants of all higher order modes in the rib region, II, are lower than that of the fundamental mode in the slab regions,I. Thus, the higher order modes will couple into the fundamental slab mode of regions I and leak out of the waveguide (become filtered) $[6,7,35]$.

The single-mode criterion for dimensions of the rib waveguide is described by

$$
\frac{a}{b} \leq \alpha+\frac{r}{\sqrt{1-r^{2}}}
$$

and

$$
0.5 \leq r \leq 1.0
$$

where $a=$ (rib width) $/ 2 \lambda$ and $b=$ (slab hight) $/(2 \mathrm{r} \lambda) . \alpha$ is a constant defined as $\alpha=0.3$ by Soref et al. [6] and $\alpha=-0.05$ by Pogossian et al. [7]. According to Soref's derivations the etched slab region must be at least half the height of the rib region i.e. $r=h / H>0.5$ while the latter is based on the experimental results obtained by Rickman et al. [36]. The Figure 2.5 shows the cross section of a rib waveguide with the parameters involved.

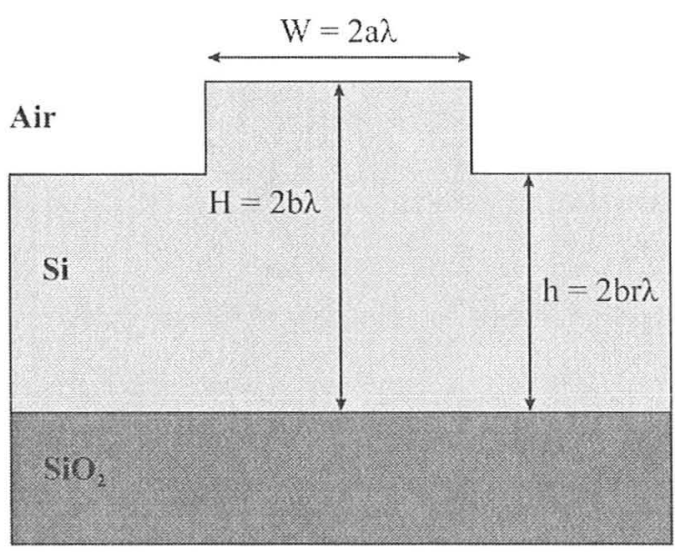

Figure 2.5: Cross section of a rib waveguide 
Here $\lambda$ is the free-space optical wavelength and $r$ is the fractional height of the side regions compared to the rib's center.

\subsubsection{Mode Properties}

Maxwell's equations and waveguide symmetries lead to modal properties and symmetries that are important components for formulating the coupled mode theory (CMT) used to model Bragg gratings.

The first set of waveguide symmetries are the reversal symmetries. For simplification, the electric field and similarly magnetic field can be separated into longitudinal and transversal components

$$
\begin{array}{r}
\mathbf{E}=\mathbf{E}_{t}+\widehat{z} \mathbf{E}_{z} \\
\mathbf{E}_{t}=\widehat{x} \mathbf{E}_{x}+\widehat{y} \mathbf{E}_{y}
\end{array}
$$

If the propagation constant $\beta$ is replaced by $-\beta$, Maxwell's equations can still be satisfied with a new solution of the form

$$
\begin{array}{ll}
\mathbf{E}_{t 2}=\mathbf{E}_{t 1} & , \quad \mathbf{H}_{t 2}=-\mathbf{H}_{t 1} \\
\mathbf{E}_{z 2}=\mathbf{E}_{z 1} & , \quad \mathbf{H}_{z 2}=\mathbf{H}_{z 1}
\end{array}
$$

Therefore, there is a corresponding backward traveling mode for every forward traveling mode, which is related through equations 2.30 . This symmetry is known as $z$-reversal symmetry. Similarly, time-reversal symmetry allows the existence of solutions in the case that sign of $t$ is reversed in the Maxwell's equations. These equations can be satisfied by a modified solution in the form of 


$$
\mathbf{E}_{2}(\mathbf{r}, t)=\mathbf{E}_{1}(\mathbf{r},-t) \quad, \quad \mathbf{H}_{2}(\mathbf{r}, t)=-\mathbf{H}_{1}(\mathbf{r},-t)
$$

Moreover, the modal orthogonality of the waveguides is the basis of CMT and can be generalized for all the $m$ and $n$ modes by either of the following equations $[37,1]$

$$
\begin{array}{r}
\iint\left(\mathbf{E}_{m t} \times \mathbf{H}_{n t} \cdot \widehat{z} d S=2 P \operatorname{sgn}(n) \delta_{|m||n|}\right. \\
\int_{-\infty}^{\infty} E_{y}^{l} E_{y}^{m} d x=\frac{2 \omega \mu}{\beta_{m}} \delta_{l, m}
\end{array}
$$

where $P$ is the power carried by the mode and $\operatorname{sgn}(\mathrm{n})$ is +1 for positive $n$ and -1 for the negative $n$. These relations state that the waveguide only supports orthogonal modes. Hence, a complete set of the corresponding modes can form a basis set to describe any arbitrary light propagation through the waveguide by their linear superposition. Furthermore, the orthogonal modes propagate independently of one another and there is no power exchange between them.

\subsubsection{Beam Propagation Method and Mode profile}

There are many numerical techniques such as finite element methods and boundary integral techniques to solve partial differential equations. The Beam Propagation Method (BPM) is one of the more widely used methods; it implements the basic techniques rapidily with optimal computational complexity. BPM is an approach to approximate the exact wave equation for a monochromatic wave and is based on factoring out the rapid phase variation $\phi$ of the field by introducing a slowly varying field $u$ via equation 2.33 such that the Helmholtz equation 2.34 is simplified 
to equation $2.35[38]$

$$
\begin{gathered}
\phi(x, y, z)=u(x, y, z) e^{i k_{r} z} \\
\frac{\partial^{2} \phi}{\partial x^{2}}+\frac{\partial^{2} \phi}{\partial y^{2}}+\frac{\partial^{2} \phi}{\partial z^{2}}+k(x, y, z)^{2} \phi=0 \\
\frac{\partial u}{\partial z}=\frac{j}{2 k_{r}}\left(\frac{\partial^{2} u}{\partial x^{2}}+\frac{\partial^{2} u}{\partial y^{2}}+\left(k^{2}-k_{r}^{2}\right) u\right)
\end{gathered}
$$

where $\phi(x, y, z)$ is the time independent part of the scalar electric field, $k$ is the medium wave number, and $k_{r}$ is the reference wave number, which represents the average phase variation of the field. Therefore, the wave equation is reduced to a first order initial value problem.

BPM employs many numerical techniques like split-step, which was one of the earliest techniques used, and finite difference method, which is the approach mostly employed to solve the integrated optics related problems.

In this method, the electric or magnetic field is computed as the field evolves along the propagation direction $(z)$ at discrete grid points in the transverse plane $(x y)$. Thus, field changes can be monitored very conveniently since the field profile may be extracted and analyzed at any point along the propagation direction[38].

\subsection{Bragg Gratings}

The ability to efficiently couple the power between the forward traveling mode and the backward traveling mode leads to important features in integrated optics like wavelength filtering, optical feedback, and broadband reflectors. This coupling can be obtained by introducing a periodic modulation in a waveguide.

One possibility for providing this modulation is to introduce periodic variation 
in the index of refraction of the dielectric waveguide. When the periodicity is confined to one dimension the arrangement is referred to as Bragg grating while in two or three dimensions photonic crystal terminology is used. These arrangements affect the propagation of the electromagnetic waves and lead to new phenomena. This is analogous to the way that the motion of the electrons in a semiconductor crystal lattice leads to an electronic bandgap or to the diffraction of $\mathrm{x}$-rays by a crystal lattice.

Bragg gratings are one of the most valuable elements in integrated optics. Their dimensions, structures, and fabrication procedures are ideally suited for integration and they can be used both as passive and active devices. Bragg gratings manipulate the propagation constant of the propagating wave through diffraction and couple waves. Input/output couplers, waveguide couplers, broadband couplers, reflectors, mode convertors, wavelength filters, and waveguide lenses are some of the passive components that are realized using gratings. Some of them are illustrated in Figure 2.6

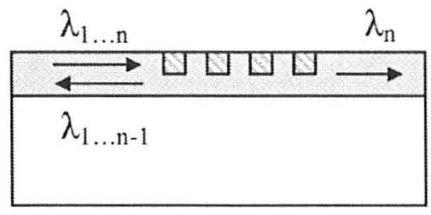

(a) Wavelength Filter

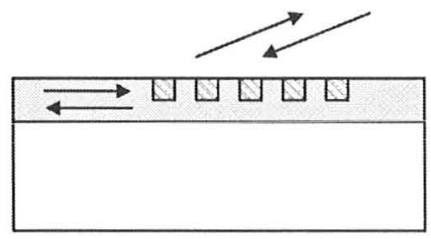

(c) Input/Output Coupler

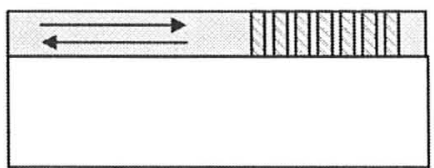

(b) Broadband Coupler

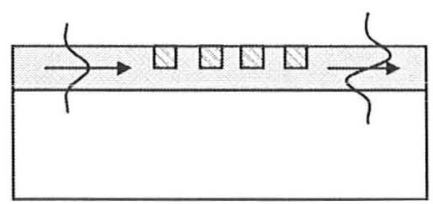

(d) Mode Converter

Figure 2.6: Passive grating components for optical circuits 
The most common and well known method to fabricate gratings is to employ photorefractive effect via UV illumination while other effects such as the acoustooptic and electrooptic effets can also be used to induce the periodic modulation in the refractive index. However, these methods can be used mostly in dielectrics and III-V materials, not in silicon, since these effects are weak or absent in silicon. Thus, the common approach for patterning silicon is physically corrugating its surface with lithography and etching processes or focused ion milling. However, retaining a planar surface is becoming important in the integrated optics as procedures like subsequent processing and wafer bonding are becoming more common in fabrication. Alternative techniques such as ion implanting can be utilized to modify the index of refraction locally while maintaining the planar surface. In the following sections the fundamentals governing devices based on Bragg gratings are explored.

\subsubsection{Fundamentals of Bragg Gratings}

A Bragg grating can be considered as a one-dimensional diffraction-based specular reflector. In order for light to be diffracted from the forward-traveling mode and couple into backward-traveling mode, the reflected light from the consecutive interfaces must interfere constructively. For incident light with vacuum wavelength $\lambda_{0}$, the Bragg period $\Lambda$ is defined by

$$
\Lambda=\frac{m \lambda_{0}}{2 n_{e f f}}
$$

where $m$ is a positive integer representing the order of the Bragg grating and $n_{\text {eff }}$ is the effective index of refraction of the mode. Considering that the first-order 
diffraction is the strongest, first-order Bragg gratings are the primary concern and therefore, $\mathrm{m}$ is set to one. Thus, the required grating period for different wavelengths and material can be determined. Since the main goal of silicon photonics is to facilitate and escalate the fabrication of integrated optical components for telecom applications, wavelengths around $1550 \mathrm{~nm}$ are of greatest interest. Approximating the effective index of refraction of the waveguide modes to that of silicon (i.e. $n=3.5$ ) and using equation 2.36 leads to the grating period of $220 \mathrm{~nm}$. The Bragg gratings considered in this thesis are formed via selective ion implantation where crystalline damage accumulates and changes the refractive index. The characteristics of these gratings will be discuss in more details later. A typical ion implanted Bragg grating is depicted in Figure 2.7.

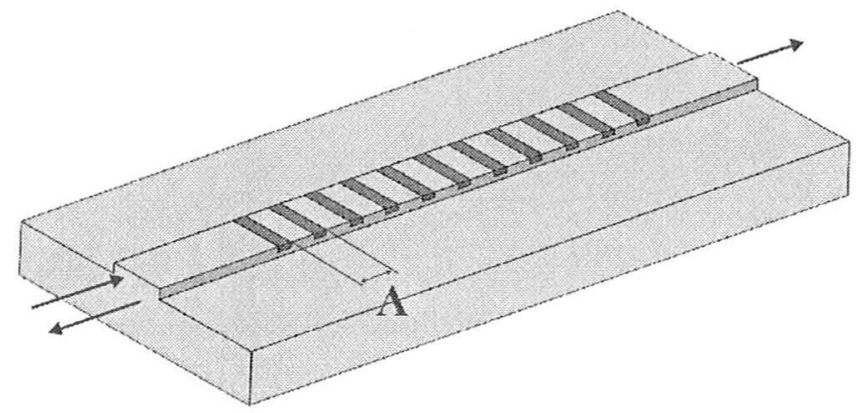

Figure 2.7: Ion implanted Bragg gratings with period $\Lambda$. The forward and backward traveling modes can be coupled under Bragg condition 
Sh. Homampour - Masters Thesis

\subsubsection{Contradirectional Coupled Mode Theory in a Periodic}

\section{Structure}

The study of wave propagation in periodic structures has been studied in many different fields including solid-state physics, crystallography, surface wave acoustics, and of course integrated optics. Many techniques are used to solve this problem and the Floquet [39] (or in solid-state physics Bloch theorem) is one of them. This method evaluates the field by decomposing it into discrete harmonics and applying Green's theorem to the components [40]. Finite-difference time domain algorithms can also be used to find the numerical solutions of this method [41]. The exact solutions of all Bragg interactions including a propagating electromagnetic wave in a Bragg grating structure can be found using this technique.

A better and more common approach is to treat the gratings or periodic variations of dielectric constant as perturbations in a $z$-invariant unperturbed structure i.e. Coupled Mode Theory. In this approach the behavior of the perturbed wave is expressed as the superposition of normal modes of an unperturbed waveguide. Thus, the coupling between the orthogonal modes of the unperturbed waveguide as the result of the perturbation is predicted. This separation is illustrated in Figure 2.8. Ray optics and diffraction theory [42] and effective index matching [43] are other alternative modeling methods.

The dielectric constant in this system can be written as

$$
\varepsilon(x, y, z)=\varepsilon_{u}(x, y)+\Delta \varepsilon(x, y, z)
$$

where $\varepsilon_{u}$ is the unperturbed part of the dielectric and $\Delta \varepsilon$ is the periodic perturbation of the dielectric in $z$ direction which is only non zero at the gratings. The 


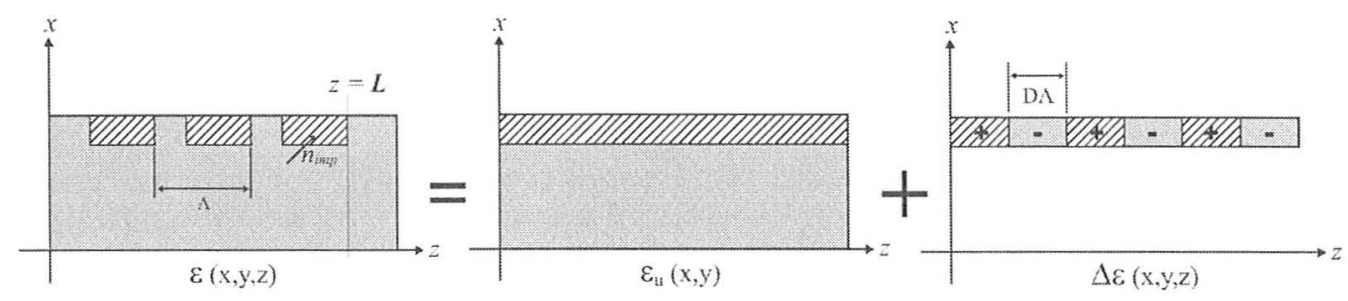

Figure 2.8: Expression of a perturbed wave in CMT. Such expression with an additional layer in the unperturbed section is called Additional Layer Method with a zaveraged index of refraction

second term multiplied by the field leads to an additional polarization field that acts as the radiation source and can be written as equation 2.38 .

$$
\Delta \mathbf{P}=\Delta \varepsilon(x, y, z) \mathbf{E}(x, y) e^{i(\omega t-\beta z)}
$$

This induced source transfers the energy between the modes, which is the premise of coupled mode theory. In the $z$-invariant case of an unperturbed waveguide, the normal modes of the propagation field can be expressed as

$$
\mathbf{E}(x, y, z, t)=\mathbf{E}_{m}(x, y) e^{i\left(\omega t-\beta_{m} z\right)}
$$

where $\beta_{m}$ is the propagation constant of the mth order mode and $E_{m}$ is the wave function of that mode. The propagation constant can be solved using equation 2.18 and expressing $n$ in terms of $\varepsilon_{u}$. Thus, any field propagating in the unperturbed waveguide can be defined as linear superposition of the normal modes with scalar modal expansion coefficients denoted as $A_{m}$ by the following expression

$$
\mathbf{E}=\sum_{m} A_{m} \mathbf{E}_{m}(x, y) e^{i\left(\omega t-\beta_{m} z\right)}
$$

The expansion coefficients are constant in the unperturbed waveguide, implying 
that there won't be any coupling or energy transfer between the modes. However, in the perturbed waveguide the second term in the right hand side of the equation 2.37 results in a $z$-dependant modal expansion coefficient and equation 2.40 becomes

$$
\mathbf{E}=\sum_{m} A_{m}(z) \mathbf{E}_{m}(x, y) e^{i\left(\omega t-\beta_{m} z\right)}
$$

where $A_{m}(z)$ accounts for the coupling between the orthogonal modes and describes the evolution of the coefficient as the wave propagates in $\mathrm{z}$ direction. The wave equation is modified to

$$
\left[\nabla^{2}+\omega^{2} \mu\left(\varepsilon_{u}(x, y)+\Delta \varepsilon(x, y, z)\right)\right] \mathbf{E}=0
$$

Substituting equation 2.41 into equation 2.42 and simplifying leads to

$$
\begin{array}{r}
\sum_{m}\left(\frac{d^{2} A_{m}}{d z^{2}}-2 i \beta_{m} \frac{d A_{m}}{d z}\right) \mathbf{E}_{m}(x, y) e^{i\left(\omega t-\beta_{m} z\right)}= \\
-\omega^{2} \mu \sum_{n} \Delta \varepsilon(\mathbf{r}) A_{n} \mathbf{E}_{n}(x, y) e^{i\left(\omega t-\beta_{n} z\right)} .
\end{array}
$$

Assuming slow amplitude variation approximation, i.e.

$$
\left|\frac{d^{2} A_{m}}{d z^{2}}\right| \ll \beta_{m}\left|\frac{d A_{m}}{d z}\right|
$$

The second ordered term in the left hand side of the equation 2.43 can be neglected. Taking the dot product of $\mathbf{E}_{k}^{*}(x, y)$ with both sides of the simplified form of equation 2.43, integrating over the whole xy plane, and employing equation $2.32 \mathrm{~b}$ 
the solution becomes

$$
\frac{2 \omega \mu}{\left|\beta_{k}\right|} \frac{d A_{k}}{d z}=\frac{\omega^{2} \mu}{2 i \beta_{k}} \sum_{n}\left(\int \mathbf{E}_{k}^{*} \cdot \Delta \varepsilon(x, y, z) \mathbf{E}_{n} d x d y\right) A_{n}(z) e^{i\left(\beta_{k}-\beta_{n}\right) z}
$$

Expanding the perturbation as a Fourier series in $\mathrm{z}$ for a periodic medium gives

$$
\Delta \varepsilon(x, y, z)=\sum_{m \neq 0} \Delta \varepsilon_{m}(x, y) e^{i m \frac{2 \pi}{\Lambda} z} .
$$

Note that $\Delta \varepsilon_{m}$ is real and that the summation is over all of the $m$ harmonics and $m=0$ is excluded here since it is considered in the unperturbed term. Defining $\kappa_{k n}^{m}$, the coupling coefficient as

$$
\kappa_{k n}^{m}=\frac{\omega}{4} \int \mathbf{E}_{k}^{*} \cdot \Delta \varepsilon_{m}(x, y) \mathbf{E}_{n} d x d y
$$

and substituting equation 2.46 into equation 2.45 the solution can be expressed as

$$
\frac{d A_{k}}{d z}=-i \frac{\left|\beta_{k}\right|}{\beta_{k}} \sum_{m} \sum_{n} \kappa_{k n}^{m} A_{n}(z) e^{i\left(\beta_{k}-\beta_{n}-m \frac{2 \pi}{\Lambda}\right) z} .
$$

The coupling coefficient determines the strength of coupling and the energy transfer between the modes. This coefficient depends on the strength of the perturbation and is only non-zero in the grating region. It is the overlap between the $k t h$ and $n t h$ modes due to the $m t h$ Fourier component of the perturbation and is also called the reflectivity per unit length of the grating. The coupling length is defined as $L=\pi / 2 \kappa$.

The equation 2.48 is the complete set of the linear differential equations, expressing the coupling between the modes. In single mode waveguides this equation 
reduces to a pair of equations describing the coupling between the two modal amplitudes of forward and backward traveling modes.

There are two important conditions for the coupling to occur: one is the phase matching condition

$$
\Delta \beta=\beta_{k}-\beta_{n}-m \frac{2 \pi}{\Lambda}=0 .
$$

This is required for resonant coupling. Otherwise, the phase dependence of the traveling wave and the source i.e. $\beta_{k}$ and $\beta_{n}$ respectively are different, and the coupling averages out to zero over the distance $\mathrm{z}$ [1]. The second condition for the coupling to happen is the conservation of the total power carried by the coupled modes.

In the contra-directional mode coupling in a single mode waveguide with periodic perturbation, equation 2.48 simplifies to

$$
\begin{aligned}
& \frac{d}{d z} A=-i \kappa B(z) e^{i \Delta \beta z} \\
& \frac{d}{d z} B=i \kappa^{*} A(z) e^{-i \Delta \beta z}
\end{aligned}
$$

where $\mathrm{A}(\mathrm{z})$ is the amplitude of the forward traveling mode and $\mathrm{B}(\mathrm{z})$ is the amplitude of the backward traveling mode, $\kappa=\kappa_{00}^{m}$ and $\kappa_{00}^{m}=\left[\kappa_{00}^{-m}\right]^{*}=\kappa$. The total power carried by these modes is conserved since

$$
\frac{d}{d z}\left[|B|^{2}-|A|^{2}\right]=0 .
$$

The effective index of the mode is defined such that

$$
\beta=n_{\text {eff }} \frac{\omega}{c}=n_{\text {eff }} \frac{2 \pi}{\lambda}
$$


thus, the phase matching condition can also be modified as

$$
\begin{aligned}
\Delta \beta & =2 \beta-m \frac{2 \pi}{\Lambda} \\
& =2\left(\beta-\beta_{0}\right) \\
& =\frac{2 n_{\text {eff }}}{c}\left(\omega-\omega_{0}\right)
\end{aligned}
$$

where $\beta_{0}=m \frac{\pi}{\Lambda}$ and $\omega_{0}$ is the center frequency where the phase matching condition is satisfied and $\omega$ is the operating frequency. To determine the behavior of the optical fields, it is sufficient to calculate the propagation constant, which can be achieved by solving equations 2.50 . The general solution to these equations is defined as

$$
\begin{aligned}
& A(z)=C_{1} e^{i(\Delta \beta / 2) z-s z}+C_{2} e^{i(\Delta \beta / 2) z+s z} \\
& B(z)=\frac{i}{\kappa} \frac{d}{d z} A(z)
\end{aligned}
$$

where $C_{1}$ and $C_{2}$ are constants to be determined by boundary conditions and $s$ is defined as

$$
s=\sqrt{|\kappa|^{2}-\left[\beta(\omega)-\beta_{0}\right]^{2}} .
$$

Assuming that the grating region is extended between $z=0$ to $z=L$, the forward traveling mode of light is expressed as $A_{0}$ at the input, and that there is no backward traveling mode at the end of the grating region $(z=L)$ i.e. $B(L)=0$ then, the particular solutions are 


$$
\begin{aligned}
& A(z)=e^{i(\Delta \beta / 2) z} \frac{s \cosh [s(L-z)]+i(\Delta \beta / 2) \sinh [s(L-z)]}{s \cosh [s L]+i(\Delta \beta / 2) \sinh [s L]} A(0) \\
& B(z)=e^{-i(\Delta \beta / 2) z} \frac{-i \kappa^{*} \sinh [s(L-z)]}{s \cosh [s L]+i(\Delta \beta / 2) \sinh [s L]} A(0)
\end{aligned}
$$

The result is the forward and backward propagating modal coefficients at any point $z$ in the grating region with the reflectance efficiency that is calculated through

$$
R=\left|\frac{B(0)}{A(0)}\right|^{2}=\frac{|\kappa|^{2} \sinh ^{2}(s L)}{s^{2} \cosh ^{2}(s L)+(\Delta \beta / 2)^{2} \sinh ^{2}(s L)}
$$

Under the phase matching condition for maximum reflectance, the above solutions simplify to

$$
\begin{aligned}
& A(z)=\frac{s \cosh [s(L-z)]}{s \cosh [s L]} A(0) \\
& B(z)=\frac{-i \kappa^{*} \sinh [s(L-z)]}{s \cosh [s L]} A(0) .
\end{aligned}
$$

For sufficiently large arguments of cosh and sinh functions in the mode powers i.e. $|A(z)|^{2}$ and $|B(Z)|^{2}$, the incident power drops off exponentially along the perturbation region due to the reflection of power. The $z$-dependent part of the solutions of the perturbed waveguides being exponentials with complex propagation constants implies that the modes will experience either exponential attenuation or amplifications depending on contribution of the amplitudes of propagation. 


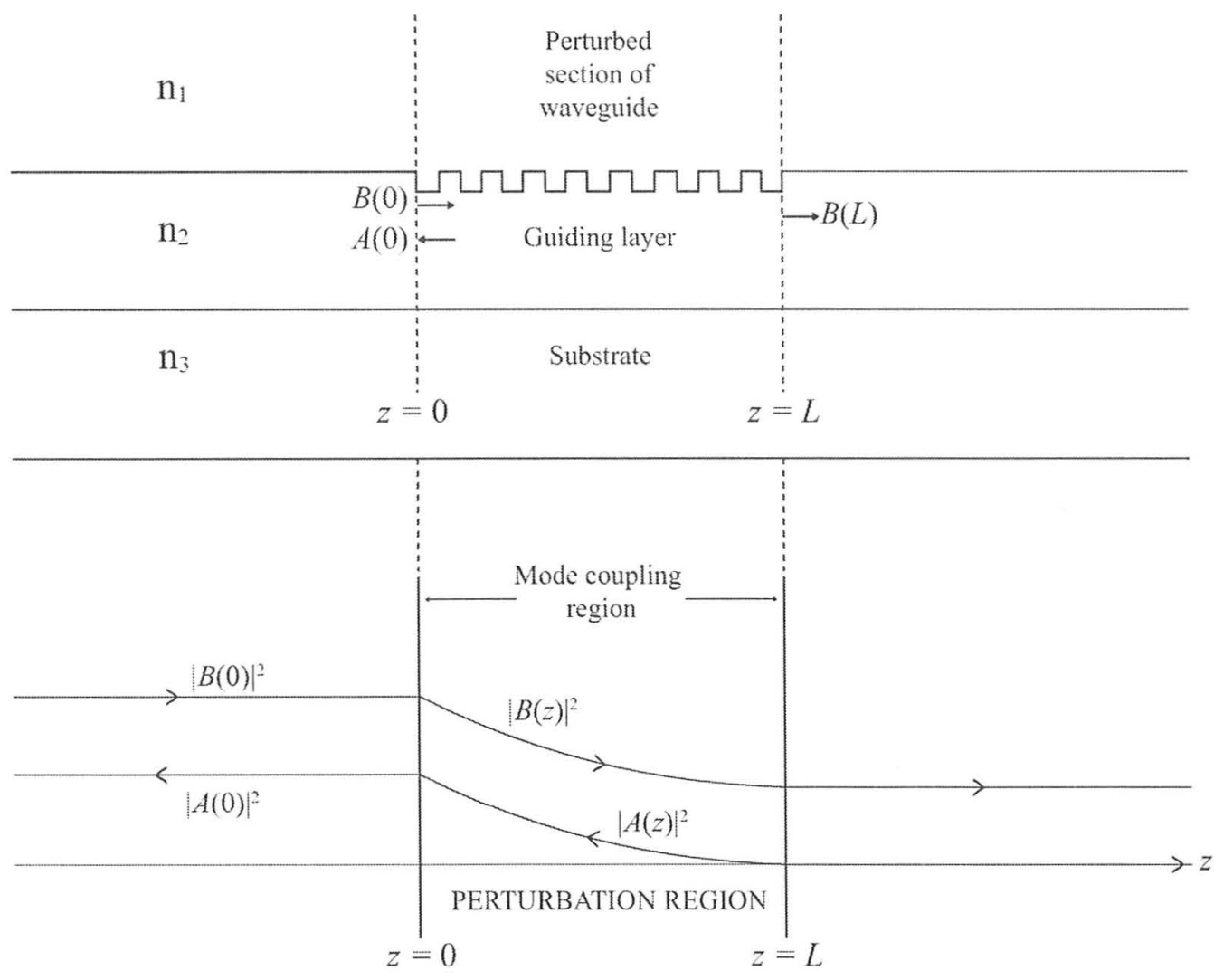

Figure 2.9: A corrugated section of the dielectric waveguide with the incident and reflected intensities inside this section [1]

$$
\beta_{1}=\Delta \beta / 2 \pm i s=\beta-\beta_{0} \pm i \sqrt{|\kappa|^{2}-\left[\beta(\omega)-\beta_{0}\right]^{2}} .
$$

Therefore, the range of frequencies where $\Delta \beta(\omega)<\kappa$ the propagation constant has imaginary part and evanescent behavior occurs. Thus, this range is called the forbidden region which is analogous to the energy band gap in semiconductors. The width of this region is defined by

$$
(\Delta \omega)_{g a p}=\frac{2 \kappa c}{n_{e f f}} .
$$




\subsubsection{Coupling Coefficient for Index Modulated Gratings}

The coupling coefficient for implanted gratings can be calculated using the method mentioned in the previous section and the dielectric constant can be decomposed into unperturbed and perturbed constituents. In general the dielectric constant is related to the index of refraction through

$$
\varepsilon(x, y)=\varepsilon_{0} n^{2}(x, y)
$$

therefore, the dielectric perturbation can be described [1] by

$$
\Delta \varepsilon(\mathbf{r}) \equiv \varepsilon_{0} \Delta n^{2}(\mathbf{r})
$$

The unperturbed dielectric constant in the region of the grating is taken to be the $z$-averaged value given by equation 2.63

$$
\varepsilon_{u}(x, y)=\left\{\begin{array}{cc}
\varepsilon_{0}\left(D n_{2}^{2}+(1-D) n_{\text {imp }}^{2}\right) & \text { grating } \\
\varepsilon_{0} n^{2}(x, y) & \text { elsewhere }
\end{array}\right.
$$

where $D$ is the duty cycle of non-implanted regions, $n_{2}$ is the index of guiding layer, and the permeability over the grating region is the $\mathrm{z}$-averaged index of refraction. Similarly, assuming square implanted regions hence, a uniform distribution of gratings in the guiding layer, the periodic modulation of the index of refraction can be expressed as a Fourier series

$$
\Delta n(x, z)=\left\{\begin{array}{cc}
\sum_{q \geq 0} \Delta n_{q}(x) \cos \left(q \mathbf{K} z+\phi_{q}\right) & \text { grating } \\
0 & \text { elsewhere }
\end{array}\right.
$$


where $|\mathbf{K}|=2 \pi / \Lambda$ and $\Delta n_{q}(x)=\Delta n_{q}$. In the case the induced index change is small $(\Delta n \ll n)$ the following approximation holds [44]

$$
\varepsilon+\Delta \varepsilon=\varepsilon_{0}(n+\Delta n)^{2} \approx \varepsilon_{0}\left(n^{2}+2 n \Delta n\right) \Rightarrow \Delta \varepsilon=2 \varepsilon_{0} n \Delta n
$$

Thus, the $\Delta \varepsilon_{q}(x)$ can be expressed as

$$
\Delta \varepsilon_{q}(x)=\Delta \varepsilon_{q}=n_{f} \varepsilon_{0} \Delta n_{q} e^{-i \phi_{q}}
$$

where $n_{f}$ is the guiding layer's refractive index. Hence, from equations 2.47 and 2.66 the coupling coefficient of TE-TE mode coupling can be verified as

$$
\kappa_{T E_{k}, T E_{n}} \approx \frac{\pi \Delta n_{q}}{\lambda} \frac{n_{f} \int_{-T}^{0} E_{k n}^{*} E_{n k} d x}{\sqrt{N_{k} N_{n}}\left[\int_{-\infty}^{\infty}\left|E_{k n}\right|^{2} d x \int_{-\infty}^{\infty}\left|E_{n k}\right|^{2} d x\right]^{1 / 2}}
$$

where $-T<x<0$ is the grating region and $N$ is the effective index of the mode. Similarly, the coupling coefficient of the TM-TM mode can be derived and expressed as

$$
\kappa_{T M_{k}, T M_{n}} \approx \frac{\pi \Delta n_{q}}{\lambda} \frac{n_{f} \sqrt{N_{k} N_{n}} \int_{-T}^{0}\left(H_{k n}^{*} / \varepsilon\right)\left(H_{n k} / \varepsilon\right) d x}{\left[\int_{-\infty}^{\infty}\left(\left|H_{k n}\right|^{2} / \varepsilon\right) d x \int_{-\infty}^{\infty}\left(\left|H_{n k}\right|^{2} / \varepsilon\right) d x\right]^{1 / 2}} .
$$

Since the second factor in the above solutions will yield to 1 for modes with same order $(k=n)$ and to zero otherwise therefore, the coupling coefficients in this case can be simplified to 


$$
\kappa_{T E_{k}, T E_{n}} \approx \kappa_{T M_{k}, T M_{n}} \approx \frac{\pi \Delta n_{q}}{\lambda} \delta_{k n}
$$

This relation indicates that the modal conversion between modes of different order cannot happen but the conversion between the modes of same order is strong specially for contra-directional coupling (of the same mode). Therefore, such structures are suitable for fabricating waveguide reflectors employed as lasers and wavelength demultiplexers.

The dielectric constant of the perturbation part can also be written in terms of the Fourier coefficients of the z-periodic perturbation as

$$
\Delta \varepsilon(x, y, z)=\left\{\begin{array}{cc}
\varepsilon_{0}\left(n_{2}^{2}-n_{i m p}^{2}\right) \sum_{m=-\infty}^{\infty} b_{m} \exp \left(-i m \frac{2 \pi}{\Lambda} z\right) & \text { grating } \\
0 & \text { elsewhere }
\end{array}\right.
$$

where the Fourier coefficients can be computed as

$$
b_{m}=\frac{\sin (m \pi D)}{m \pi}
$$

leading to the coupling coefficient of a single mode rib waveguide in terms of the duty cycle of the non-implanted regions

$$
\kappa_{00}^{(m)}=\frac{\omega \varepsilon_{0}}{4 m \pi} \sin (m \pi D) \iint_{\text {grating }} E_{0}^{*} \cdot\left(n_{2}^{2}-n_{\text {imp }}^{2}\right) E_{0} d x d y
$$




\subsubsection{Coupling Coefficient for Relief Gratings}

The dielectric perturbation of a relief grating with a rectangular cross section can be described by

$$
\Delta \varepsilon_{q}(x)=\Delta \varepsilon_{q}\left\{\begin{array}{cc}
\varepsilon_{0}\left(n_{f}^{2}-n_{c}^{2}\right) b_{m} & -h<x<h \\
0 & \text { elsewhere }
\end{array}\right.
$$

with $b_{m}$ as the Fourier coefficients of the z-periodic perturbation and $n_{f}$ and $n_{c}$ as the guiding layer and cover layer index of refraction. The perturbation is considered as a square wave with unitary amplitude with Fourier coefficient computed as

$$
b_{m}=\frac{\sin (q a \pi)}{q \pi}
$$

with $q \neq 0$ and $0<a<1$. Substituting equation 2.73 into equation 2.47 leads to the following relation describing the coupling coefficient between two TE modes of a single mode rib waveguide

$$
\kappa_{T E_{k}, T E_{n}}=\frac{\pi}{\lambda} \frac{\left(n_{f}^{2}-n_{c}^{2}\right)}{\sqrt{N_{k} N_{n}}} \frac{\sin q a \pi}{q \pi} \frac{\int_{-h}^{h} E_{k n}^{*} E_{n k} d x}{\sqrt{\int_{-\infty}^{\infty}\left|E_{k n}\right|^{2} d x \int_{-\infty}^{\infty}\left|E_{n k}\right|^{2} d x}} .
$$

A similar expression is found for the coupling coefficient of TM modes as a function of $\mathrm{H}$ field. Setting $q=1$ in equation 2.75 leads to the possibility of coupling between different order modes and actually higher coupling coefficient for higher order modes. The latter is due to the higher penetration of high order modes at the coupling region. A better approximation for the coupling coefficient 
Sh. Homampour - Masters Thesis

Engineering Physics, McMaster University

Chapter 2. Background Theory

is obtained when the depth of the gratings is much smaller than the thickness of the guiding layer where the electric/magnetic fields in the numerator can be considered constant with their value being that of the field at $x=0$.

Therefore, coupling between different modes can happen in relief gratings while it is much weaker in index modulated gratings. This difference is due to the uniform distribution of the periodic modulation in the former while it is localized in the surface of the waveguide in the latter case $[21,44]$.

\subsubsection{Broadband Bragg Grating Couplers on SOI}

The majority of the work described in this thesis deals with Bragg gratings that are fabricated in single mode SOI rib waveguides and designed to act as very narrowband retroreflectors. In this section we discuss another class of grating structures that could be used in a quite different application, but have very much in common with the rib waveguide retroreflectors in terms of fabrication methods. The gratings considered in this section are different in two important aspects. First, they are intended to couple light into an SOI waveguide from a free-space optical beam that is incident on the silicon surface from above. Second, the waveguide is a highly multi-mode slab waveguide, not a single-mode rib guide. The application that motivates this study is photovoltaics, in particular possibility of enhancing the long wavelength response of silicon solar cells.

As industry progresses towards more environmentally friendly technologies, energy production and the energy needs of futures generations are critically important. This is driving great interest in developing improved solar cells with lower cost and higher efficiency. Silicon is the most developed material for solar cells and is relatively low cost. One approach to improving its efficiency is to use light trapping 
Sh. Homampour - Masters Thesis

Engineering Physics, McMaster University

Chapter 2. Background Theory

techniques to enhance its optical absorption at long wavelengths that do not normally contribute to any photoresponse $[45,46]$.

Light trapping techniques involve redirecting light so that it propagates more or less parallel to the active region of the $p$ - $n$ diode rather than perpendicular to it. This approach is equivalent to increasing the cell thickness with reduced recombination losses [45]. Roughened front surfaces with sandblasted substrates [47], Bragg reflector rear mirrors [48], and textured photonic crystals (TPC) [46] are some of the methods used to accomplish this. In addition, the use of broadband grating couplers on silicon-on-insulator material has recently been demonstrated $[45,46]$.

Grating coupling between free-space beams and single-mode rib waveguides is highly developed and widely used. Gratings can be placed anywhere on the chip without edge constraints and be integrated monolithically to an optical circuit. Moreover, they provide good coupling efficiency regardless of the waveguide thickness. This efficiency remains constant since the coupler is part of the waveguide so it is not altered by the changes in the ambient. These couplers have the ability to excite only one guiding mode of the structure which is an important advantage $[21,49,50]$.

The grating couplers produce a phase matching between a particular waveguide mode and an unguided optical beam incident on the surface of the waveguide at specific angle of incidence. The periodic nature of the gratings perturbs the waveguide modes and introduces a set of spatial harmonics with propagation constants of

$$
\beta_{\nu}=\beta_{0}+\frac{\nu 2 \pi}{\Lambda}
$$


where $\nu=0, \pm 1, \pm 2, \ldots, \Lambda$ is the periodicity of the grating, and $\beta_{0}$ is the propagation constant of a guided mode without the grating. In this case, one of the space harmonics produced by the grating is synchronized with the propagation constant of a guided mode in the waveguide i.e. $\beta_{0}$. Hence, this guided mode component is excited and continues to propagate beyond the grating region and other waves are yielded by the reflection and transmission of the input beam. The grating coupler can be used to selectively transfer energy from an optical beam to a particular waveguide mode by properly choosing the incident angle of the beam. The relating equation for $\nu$ th order of space harmonic is

$$
n_{i} k_{0} \sin \left(\theta_{i}\right)=\beta_{\nu}=\beta_{0}+\frac{\nu 2 \pi}{\Lambda}
$$

where $\mathrm{n}_{i}$ is the index of refraction of the medium incident optical beam is propagating which in this case would be air and $\theta_{i}$ is the incident angle.

The details of coupling phenomena are complex and depend strongly on the cross-sectional shape of the grating bars and their spacing. In general the efficiency of the grating with symmetric profile is less than the ones with asymmetrical or blazed profile since in the former type a great part of the incident energy transmits through the guiding layer and is lost in the substrate [34]. The couplers are polarization sensitive for instance, the coupling efficiency of TM polarization can be 21 $\mathrm{dB}$ lower than that of TE polarization [51]. The grating couplers can be fabricated to enhance the coupling efficiency [50], be broadband [49] or both [51]. Employing shallow gratings in a waveguide with a width much larger than its height can yield to broadband coupler with large coupling strength [51]. The grating's uniformity, buried oxide layer's thickness and addition of reflector layers to confine the light 
Sh. Homampour - Masters Thesis

Engineering Physics, McMaster University Chapter 2. Background Theory

better have major influence on the efficiency of the device.

The grating couplers described above are designed to couple a beam of monochromatic light at a specific angle of incidence to the unique propagating mode in a single mode rib waveguide. For solar cell applications, however, one wants to couple input light from all angles of incidence and over a wide spectral range into a highly multimode, relatively thick, planar SOI layer. This would appear to be inconsistent with equation 2.77 above. However, the presence of a very large number of modes, all with different effective indices, makes coupling possible over a wide range of input angles and wavelengths. The spectral region of interest in references [45] and [46] was around $\lambda=1.0 \mu \mathrm{m}$ where the photon energy is only slightly above the bandgap energy of silicon and the small absorption coefficient makes conventional solar cells very inefficient. However, the same light trapping techniques could allow silicon solar cells to harvest energy from sub-bandgap photons if defect engineering techniques are used to induce optical absorption in the 1.2 to $1.6 \mu \mathrm{m}$ range [52].

To estimate the effectiveness of coupling from a free space beam to a multimode planar waveguide, a simulation was carried out using FullWave software from Rsoft Design Group. A typical result is shown in Figure 2.10. A beam of light is directed from below onto a $5 \mu \mathrm{m}$ thick silicon layer. A grating was patterned on the first surface of the silicon slab with pitch of $520 \mu \mathrm{m}$ and $50 \%$ duty cycle. An input of $10 \mu \mathrm{m}$ wide was launched at $\mathrm{x}=0$ with monitors placed at $\mathrm{x}=20,50,100,150$, 200, 250, 300, and $350 \mu \mathrm{m}$.

Without the grating, the beam would simply be transmitted through the slab and no light would be trapped within it. The presence of the grating causes a fraction of the optical power from the beam to the slab couple slab guided light back into free space, thus providing a loss mechanism that competes with optical absorption. As 


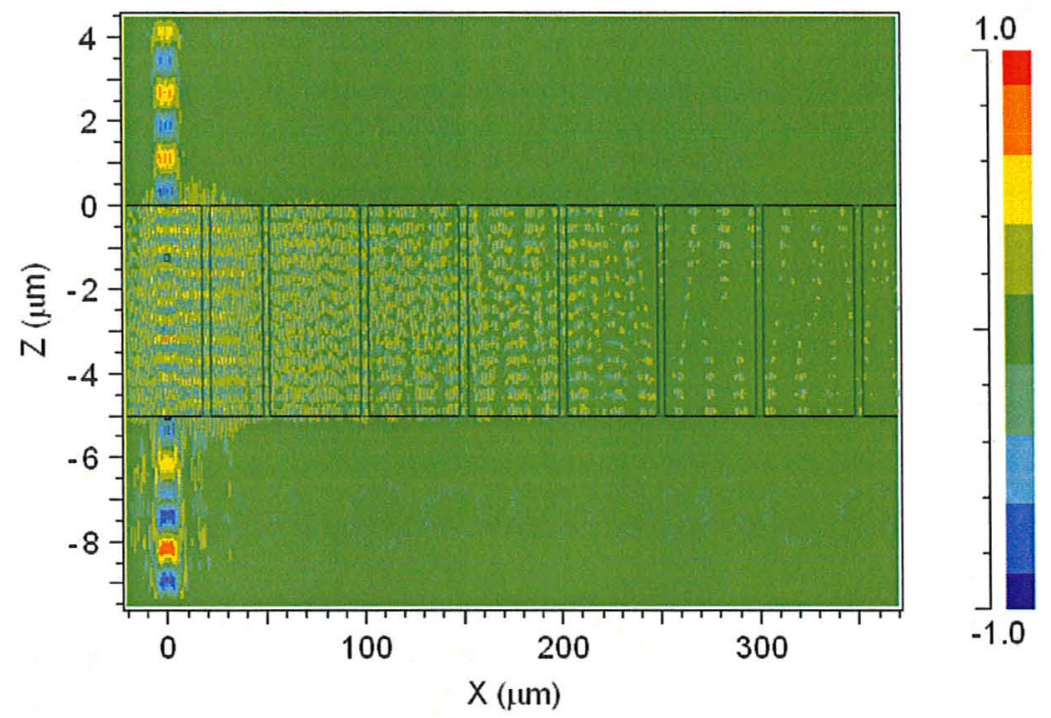

Figure 2.10: The contour map of the power propagation of TE polarized beam in gratings

illustrated in Figure 2.11, the simulations indicate that the decay length for this loss mechanism is on the order of $100 \mu \mathrm{m}$.

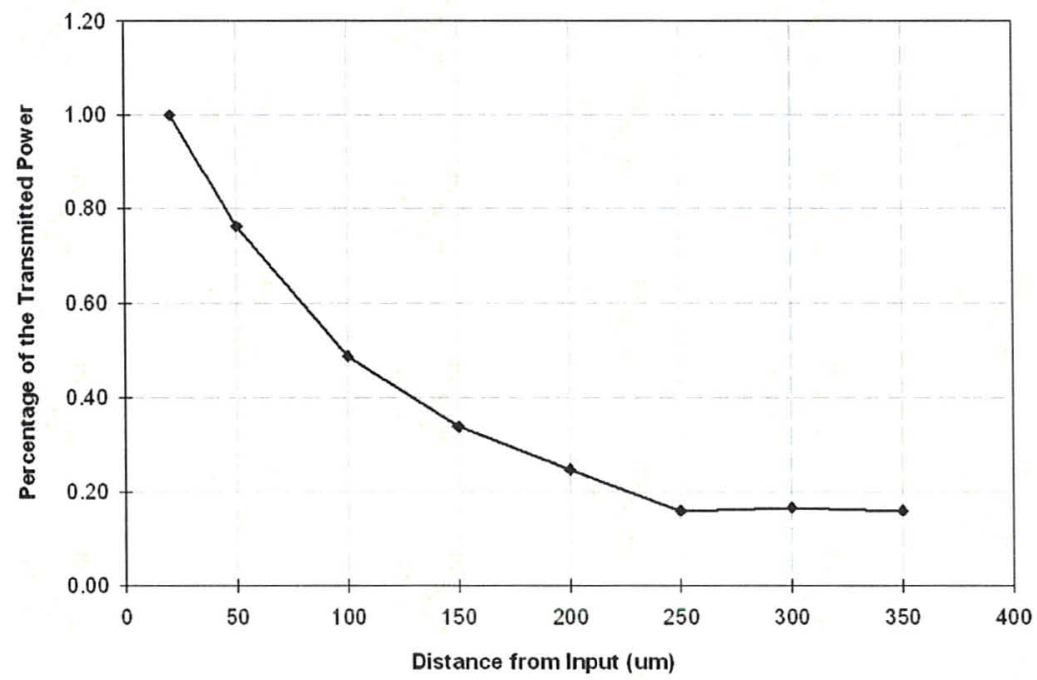

Figure 2.11: The plot of the power percentage of the TE mode vs. the distance under grating

We obtained experimental results that are consistent with these simulations. The 
Sh. Homampour - Masters Thesis

Engineering Physics, McMaster University

Chapter 2. Background Theory

fabrication details are given in the section 3.6 and the measured results are given in 4.3 .

\subsection{Ion Implantation in Silicon}

Ion implantation is ubiquitous in the fabrication of silicon integrated circuits and is the primary method used for the doping of semiconductors. The ability of ion implantation to precisely control the dopant dose and concentration profile makes the technique suitable for fabrication processes which require strict control of impurity introduction. Moreover, ion implantation is generally performed at roomtemperature which makes it compatible with doping masks with low thermal stability; such as photoresist [5]. In addition to the introduction of dopant, high dose inert ion implantation may be used for defect engineering. For instance, selfimplantation of silicon ions to doses $>1 \times 10^{15} \mathrm{~cm}^{-2}$ renders silicon amorphous. This is important in the formation of shallow junctions (through the elimination of subsequent dopant ion channeling) and in the case of silicon photonics offers a mean to selectively alter the refractive index. Amorphous silicon posses a higher index of refraction in comparison to crystalline silicon, making it a potential candidate as a core material for optical circuits [53]. The additional functionality of the silicon photonic devices due to selective self-amorphization is of interest in this work, hence the conditions for the creation of self-amorphization by ion implantation and the effects of the defects produced during the process are explored in this section. 
Sh. Homampour - Masters Thesis

Engineering Physics, McMaster University Chapter 2. Background Theory

\subsubsection{Fundamentals of Ion Implantation}

In order to describe the distribution of implanted ions in silicon a detailed atomicscale description of the events along the ion trajectory is required. The entrance of an ion into a lattice of crystalline silicon leads to its energy loss along its trajectory caused by both nuclear and electronic scattering. The rate of this loss is given by equation 2.78

$$
\frac{d E}{d x}=-N\left[S_{n}(E)+S_{e}(E)\right]
$$

where $N$ is the target atomic density $\left(5 \times 10^{22} \mathrm{~cm}^{-3}\right.$ for silicon) and $S_{n}(E)$ and $S_{e}(E)$ are the nuclear and electronic stopping powers in $\mathrm{eV} \mathrm{cm}^{2}$ respectively [2].

The nuclear energy loss is due to the elastic scattering of ions from target atoms and depends strongly on the ion energy. On the other hand, the electronic stopping is inelastic and the result of two effects: the first is the drag force that a moving ion experiences while propagating in the implantation target and the second is the overlap between wavefunctions of electrons of the ion and the lattice atom. Both effects depend on the velocity of the incident ion.

At relatively high ion energies, the nuclear energy loss is small due to the small interaction time and reduced cross section between the fast particle and scattering nucleus, thus electronic energy loss is dominant. For energies approaching the threshold for vacancy creation (approximately $15 \mathrm{eV}$ ), nuclear energy loss dominates. By implication this occurs close to the end of the ion range where most of the energy of the ion is lost and the drag force that is proportional to the velocity of the ion is negligible. The nuclear stopping power is also dependent upon the atomic $Z$ number of the ions. Hence, light ions with high velocity experience relatively 
large fractions of electronic stopping power while heavy ions moving slowly lose energy primarily through nuclear stopping.

The ion distribution can be described statistically due to the random nature of the ion implantation process and the high number of the implanted ions (i.e. greater than $10^{12} \mathrm{~cm}^{-2}$ ). The distribution may be modeled to the first order by a symmetric Gaussian defined by

$$
C(x)=C_{p} \exp \left(-\frac{\left(x-R_{p}\right)^{2}}{2 \Delta R_{p}^{2}}\right)
$$

where $C_{p}$ is the peak distribution, $R_{p}$ is the average projected range normal to the surface, and $\Delta R_{p}$ is the standard deviation or straggle about the range. The peak distribution of the implant is defined by

$$
C_{p}=\frac{Q}{\sqrt{2 \pi} \Delta R_{p}}
$$

where $Q$ is the implantation dose. When implantation through a mask window is of concern instead of simple blanket implantation the lateral straggle becomes important. Lateral straggle indicates the number of ions scattered at the edge of the window, thus, a two-dimensional projection must be considered. This distribution is often assumed to be the product of the vertical and lateral distributions and is expressed as

$$
C(x, y)=C_{\text {vert }}(x) \exp \left(-\frac{y^{2}}{2 \Delta R_{l}^{2}}\right)
$$

where $\Delta R_{l}$ is the lateral straggle. The lateral straggle profile is very difficult to quantify experimentally but can be described statistically by a Gaussian distribution. However, the description of detailed atomic-scale events that occur along the 
Sh. Homampour - Masters Thesis

Engineering Physics, McMaster University Chapter 2. Background Theory

ion trajectory is considered as the only viable method for accurately modeling of the ions final distribution in crystalline silicon. For such accurate profiles both nuclear and electronic stopping may be simulated using Monte Carlo methods [2, 54].

\subsubsection{Amorphization of Silicon and Damage Profile}

The interaction and energy transfer between the implanted ions and lattice atoms results in structural modification of the lattice. The ion-atom collisions can produce vacancy-interstitial pairs in the lattice providing the ion energy is greater than a threshold ${ }^{5}$; otherwise, these collisions create lattice vibrations leading to local heat generation. In the case where the imparted energy to the lattice atom is greater than the required energy for production of V-I (Frenkel) pair [55], a cascade of displacements of lattice atoms is created. Such additional events are referred to as target recoils and greatly accelerate defect production and eventual amorphization of the silicon target. Although the majority of the ion induced defects are located within the ions path near the end of the range, the short range migration of the defect is possible even at room temperature. In general, implantation at low energy and temperature helps retain a greater fraction of the induced damage. The damage cascades from individual ion tracks tend to overlap and aggregate until the crystalline state of the silicon lattice becomes highly unstable. At some point, an amorphous silicon (a-Si) state becomes thermodynamically favorable hence, crystalline silicon (c-Si) transforms to a-Si state [56].

The mass of the ion species, the substrate's temperature, the dose and dose rate of the implanted ions all play an interdependent role in amorphization [54]. In order to obtain a continuous amorphous layer in a crystalline silicon substrate the implan-

\footnotetext{
${ }^{5}$ Ions energy should be about $15-20 \mathrm{eV}$
} 
tation dose must be greater than a threshold dose, otherwise, the implanted ions simply introduce isolated damage clusters and localized amorphous silicon. For the self-amorphization of silicon doses on the order of $10^{15} \mathrm{~cm}^{-2}$ produce amorphous layers when the implantation is performed at liquid nitrogen temperatures [57]. Many different modeling approaches have been suggested for simulating the introduction of ion implantation damage such as Molecular Dynamics (MD) [58], analytical models based on linear Boltzman transport theory [59], and Binary Collision Approximation (BCA) [60]. In the MD model the movement of atoms as a function of time and their interactions with all of their neighboring atoms are considered. This approach provides an accurate result for low energy processes but the calculations are time consuming. The second approach (Boltzman transport theory) describes the scattering process by the changes in the distribution of the statistical momentum. Finally, in the BCA model only collisions amongst moving and stationary atoms in the displacement cascade are of concern [61]. The BCA approach is the basis of the simulator called SSUPREM4 which is part of the ATHENA 2D process simulation software used for this work.

In order to form a suitable implanted grating for the telecommunication range i.e. incident light with a wavelength at or around $1.55 \mu \mathrm{m}$, the gratings should have a period of $220 \mathrm{~nm}$ which requires an implantaion mask with windows of width $110 \mathrm{~nm}$. To minimize the overlap between the implantation damage from adjacent windows and ensure the existence of the required modulation depth for implanted gratings, the lateral implant damage from any window should be half of the distance between the teeth i.e. it should be less than $55 \mathrm{~nm}$. The limitation on the lateral straggle of the implantation damage adds a constraint on the implantation damage depth due to their proportionality to the implantation energy. This then requires a 
compromise in process strategy with a low energy implantation ensuring minimal damage overlap between masking windows, but a higher energy producing a greater depth of modulation from the resulting grating structure. Using the SSUPREM4 simulation routine the maximum implant energy for $\mathrm{Si}^{+}$ions meeting the lateral damage constraints was determined to be up to $60 \mathrm{keV}$ for a dose of $2 \times 10^{15} \mathrm{~cm}^{-2}$ at liquid nitrogen implantation temperatures, while for the $50 \mathrm{keV}$ implant energy, the amorphous silicon depth was estimated to be $155 \mathrm{~nm}$ [62].

\subsubsection{Damage-Induced Refractive Index Modification and Loss}

Inert ion implantation leads to a change in the refractive index of silicon through the introduction of damage to the lattice. The index of refraction (both the real and imaginary parts) increases proportionally with the implanted ion dose and defect concentration until an amorphous layer is completely formed at which point the refraction index reaches a saturation value. The real part of the refractive index of amorphous silicon has been determined to be approximately 0.3 greater than that of the crystalline silicon for near infrared wavelength [53, 63].

Moreover, infrared ellipsometry measurements indicate that two optical states of amorphous silicon are produced by implantation. One is the as-implanted state where the index of refraction reaches the maximum of 0.3 ; the other is the state induced by post-implantation thermal annealing at temperatures of a few hundred degrees Celsius (such that solid phase regrowth does not occur) where the refractive index is between that for as-implanted (amorphous) silicon and the crystalline silicon states.

Several hypothesis have been suggested to explain the underlying physical mechanism resulting in the modification of the index of refraction by amorphization. The 
atomic density of the amorphous silicon was suggested to be greater than that of the crystalline silicon and the reason for the increase in the index. However, it was experimentally shown by Custer et al. that this density is approximately $1.8 \%$ lower than the density of the crystalline silicon [64].

The atomic strain was suggested as another explanation by Zammit et al.. The large degree of the lattice strain in the as-implanted induced amorphous silicon due to the point defect presence, the structural relaxation and reduction of the strain accompanied by evolution of point defects after thermal annealing and increase of the optical bandgap were some of the reasonings [65]. Similar explanations were suggested after Raman scattering and infrared reflectivity studies by Roorda et al. [66]. However, after low temperature annealing the index of refraction of the amorphized silicon is still higher than that of the crystalline silicon although amorphous silicon reaches a thermally stable state and many of the point defects are annealed. Thus, the induced strain by point defects could not be considered as the sole reason of the modification of the index of refraction.

Introduction of dangling bond defects during ion implantation which have polarization abilities was also considered irrelevant [67]. The application of the Drude or Lorentz-Lorentz dielectric equation determined that dangling bonds increase the bond polarization by $28 \%$ causing an increase in the index of refraction [53]. In summary, a reason for the modification of index of refraction due to amorphization has not be reached and likely requires an explanation involving a combination of all of the above reasons.

Another consequence of the creation of damaged crystalline or amorphous layers is the modification of the electronic structure of silicon which leads to variation of the band-edge and introduction of optical absorption in the sub-gap region which 
results in an increase in the optical absorption coefficient. The damaged crystalline state consists of various point defects and point defect complexes that introduce electronic states in the band gap. As the defect concentration increases, the band tail increases which extends into the gap and broadens the bands. Thus, absorption edge shifts towards smaller energies and the near-edge absorption increases. This later effect is related to the increase of the real component of the index of refraction $[53,68,69]$.

One of the most prominent defects in silicon at room temperature is the divacancy which has an associated absorption peak previously observed at $1.8 \mu \mathrm{m}$ with its absorption tail extending to telecom wavelengths around $1.5 \mu \mathrm{m}$ [70]. The absorption peak disappears at high doses which is known to be associated with completion of amorphization of the implanted layer in silicon. Moreover, the concentration of the divacancies first increases with implantation till it reaches a peak and then decrease till the amorphization is complete. The completion of amorphization is accompanied with a sudden increase in absorption at all wavelengths of interest $[68,71]$. The divacancies, other primary defects, and dangling bonds all contribute to the absorption losses, with relative contributions dependent on the proximity of the lattice to the amorphous state. Loss effects can be eliminated to some extend by annihilating the defects by high temperature thermal annealing (usually at temperatures above that used for solid phase epitaxy $\sim 650^{\circ} \mathrm{C}$ ). 


\subsection{Modeling Implanted Bragg Gratings in}

\section{Waveguides}

The dependence on a number of physical properties, such as grating depth and duty cycle, on the performance of a Bragg grating requires that significant care must be taken when choosing implantation conditions such as dose and energy when forming as integrated grating via ion implantation. The grating strength is directly related to the degree of overlap between gratings and propagation mode profile thus, rib dimensions should be chosen to ensure the strength is adequate. In the case of implanted Bragg gratings the implantation depth is on the order of $150 \mathrm{~nm}$ (as was shown in section 2.3.2) and using CMT the reflected grating strength can be computed as a function of silicon device thickness. The calculations show that a $2 \mu \mathrm{m}$ SOI device layer provides adequate modal overlap and also a maximum reflected grating strength when the grating depth is $150 \mathrm{~nm}$ [62]. Greater slab heights lead to lower reflected power and while coupling losses increase as the slab height is reduced.

The effect of duty cycle may be investigated by varying the index of refraction of the additional layer in equation 2.63 and the coupling coefficient for the TE and TM polarizations can be computed as a function of duty cycle using equation 2.72. The mode profile of the fundamental mode in the unperturbed SOI waveguide is determined by implementation of BPM iterative mode solver. As a result, the coupling coefficients are shown to be polarization dependent and the difference between the corresponding coupling coefficients is very large at particular duty cycles. The larger overlap between the modal profile and gratings in TE polarization leads to such differences. 
The coupling coefficient is also a function of the implant depth. As the implant depth is increased, the maximum coupling coefficient shifts away from a 50/50 duty cycle, attributed to the preferential shift of light towards the volume of the waveguide (damaged) with the higher index of refraction. The increase of implant depth, raises the coupling coefficient and thus, reduces the coupling length. Although this is beneficial in terms of device packing density, it also leads to an increasing loss due to the implant damage.

\subsubsection{Gratings with Loss}

Equations 2.56a describe a real valued perturbation case where medium gain or loss is not considered. However, ion implanted gratings make the situation more complicated by introducing loss in the medium as described previously. Numerous methods have been investigated to explain the gain and loss mechanisms in the periodic medium due to the importance of Bragg gratings in DFB lasers [72, 73].

Assuming a complex index of refraction perturbation and taking into account loss, the previously stated perturbed dielectric constant can be modified to

$$
\Delta \varepsilon(x, y, z)=\varepsilon_{0} \Delta n^{2}+i \frac{2 \Delta \gamma \Delta n}{k_{0}}
$$

where $\Delta \gamma$ is the exponential loss coefficient with $\gamma$ being negative to represent material loss [74]. Following the previous assumptions i.e. having a periodic and square wave function perturbation, the perturbation part of the dielectric constant after Fourier series expansion becomes

$$
\Delta \varepsilon(x, y, z)=\varepsilon_{0}\left[\left(\Delta n^{2}\right)+i \frac{2 \Delta \gamma \Delta n}{k_{0}}\right] \sum_{-\infty}^{\infty} b_{m} \exp \left(-i m \frac{2 \pi}{\Lambda} z\right)
$$


which leads to the modified coupling coefficient

$$
\kappa_{00}^{(m)}=\frac{\omega \varepsilon_{0}}{4 m \pi} \sin (m \pi D)\left[\left(\Delta n^{2}\right)+i \frac{2 \Delta \gamma \Delta n}{k_{0}}\right] \iint_{\text {grating }} E_{0}^{*} \cdot E_{0} d x d y
$$

where $\Delta \gamma=\gamma_{2}-\gamma_{i m p}$. Thus, the implanted damage introduces a complex coupling coefficient where gain/loss coupling can occur. The latter is the principle behind the gain coupled distribution feedback lasers. Accounting for the loss in the coupled equations 2.50 , they are modified by addition of loss components

$$
\begin{aligned}
\frac{d}{d z} A & =-i \kappa B(z) e^{i 2 \Delta \beta z}+\gamma_{u} A \\
\frac{d}{d z} B & =i \kappa^{*} A(z) e^{-i 2 \Delta \beta z}-\gamma_{u} \beta
\end{aligned}
$$

with $\gamma_{u}$ being the modal loss of the propagating mode due to the ion-induced damage. Substituting

$$
\begin{aligned}
& A(z)=A^{\prime}(z) e^{\gamma_{u} z} \\
& B(z)=B^{\prime}(z) e^{-\gamma_{u} z}
\end{aligned}
$$

into equations 2.85 results in

$$
\begin{aligned}
\frac{d}{d z} A^{\prime} & =-i \kappa B^{\prime}(z) e^{i 2\left(\Delta \beta+i \gamma_{u}\right) z} \\
\frac{d}{d z} B^{\prime} & =i \kappa^{*} A^{\prime}(z) e^{-i 2\left(\Delta \beta+i \gamma_{u}\right) z} .
\end{aligned}
$$

This set of equations are in the exact form of the coupled equations determined previously. Thus, solutions can be obtained by replacing $\Delta \beta$ with $\left(\Delta \beta+i \gamma_{u}\right)$. These solutions are given by 


$$
\begin{aligned}
& A(z)=e^{i(\Delta \beta / 2) z} \frac{s \cosh [s(L-z)]+i\left[\left(\Delta \beta+i \gamma_{u}\right) / 2\right] \sinh [s(L-z)]}{s \cosh [s L]+i\left[\left(\Delta \beta+i \gamma_{u}\right) / 2\right] \sinh [s L]} A(0) \\
& B(z)=e^{-i(\Delta \beta / 2) z} \frac{-i \kappa^{*} \sinh [s(L-z)]}{s \cosh [s L]+i\left[\left(\Delta \beta+i \gamma_{u}\right) / 2\right] \sinh [s L]} A(0)
\end{aligned}
$$

In this case, $s$ is given by

$$
s^{2}=|\kappa|^{2}+\left(\gamma_{u}-i \Delta \beta\right)^{2}
$$

therefore, the amount of loss can be estimated. For instance, for the implant depth of $150 \mathrm{~nm}$ and unannealed 50keV implant the modal loss of $\gamma_{u}=2.05 \mathrm{~cm}^{-1}$ and that of $\gamma_{u}=2.76 \mathrm{~cm}^{-1}$ for $60 \mathrm{keV}$ implant is estimated.

The grating strength is an increasing function of $|\kappa| L$. In the lossless medium it saturates to a reflected value approaching hundred percent while in the medium with absorption loss the maximum reflected power saturates to a fraction of the input power.

As the grating length $L$ increases the shape of the spectral response deviates from a "sinc" to a more "box-like" function with squeezing sidelobes. Moreover, the transmitted power decreases with an increase in grating length and the reduction is very drastic for long gratings. Thus, it is beneficial to normalize the spectral plots to their transmitted power to ease the determination of the shape of the transmitted power.

The deviation of the spectral response to a box-like function shape also happens as the absorption coefficient increase. The modal absorption coefficient is directly 
Sh. Homampour - Masters Thesis

Engineering Physics, McMaster University

Chapter 2. Background Theory

related to the implantation depth and thus the deep implants result in the change in the shape of the response. Therefore, care must be taken to minimize additional and unnecessary losses while the index of refraction is increased due to amorphization. Low temperature annealing to eliminate the point defects and other low energy defects is one of the common methods to reduce the excess optical absorption. In [53], losses on the order of $115 \mathrm{~cm}^{-1}$ were achieved after annealing at $500^{\circ} \mathrm{C}$ for 1 hour while retaining an index increase of about 0.3 .

\subsubsection{Propagation Losses due to Implantation}

The beam propagation simulations can be used to verify the dependence of transmitted power to the implanted grating depth and mode polarization. The TE polarization exhibits greatest loss which is in agreement with the greater modal overlap of this polarization with the grating region. A difference of thirty percent in the transmitted power for grating lengths of $2400 \mu \mathrm{m}$ and implant depths of 200 and $250 \mathrm{~nm}$ is observed [62]. The strong dependence of the grating strength and that of the propagation loss on the grating depth makes the chosen grating depth very important. Thus, it has to be chosen in this work to both maximize the coupling coefficient and minimize the propagation loss. Grating depths on the order of 150 $\mathrm{nm}$ seem to adhere to the restrictions present in our case. 


\subsection{Thermal Tuning of The Resonant Wavelength of}

\section{Bragg Gratings}

Although silicon based integrated photonic devices have the advantages of low cost, well known fabrication processes, and small propagation loss, as with all semiconductor based integrated circuit fabrication, variation in fabrication dimensions often lead to resonant devices which may not necessarily meet required specification. Therefore, it is often desirable to maintain the capability to fine tune resonance conditions.

The operation of integrated optical controllers are based on the electrooptic, thermooptic, and all optical effects in silicon. However, the electrooptic effect cannot be used practically due to the lack of inversion symmetry in silicon. The large thermo-optic coefficient and good thermal conductivity of the silicon permits the ability of tuning the resonant wavelength thermally. Thermal tuning is widely used since it can modify the index of refraction without adding optical losses. For instance, photonic crystal microcavities [75], microring filters [76], Hybrid semiconductor-dielectric filters [77], and recently surface relief Bragg reflectors [78] can be tuned using the thermooptic effect in silicon. Therefore, thermal tuning

can be employed on the Bragg gratings in the present study to tune the reflected wavelength.

Considering the equation for the Bragg grating period

$$
\Lambda=\frac{m \lambda_{0}}{2 n_{e f f}}
$$

for the fundamental mode i.e. $\mathrm{m}=1$ and taking the derivative of the equation 
with respect to temperature yields

$$
\frac{d \lambda}{d T}=2 \Lambda \frac{d n}{d T}+2 n \alpha \Lambda
$$

where $\alpha$ is the thermal expansion coefficient. The temperature dependence of the effective index being the same as that of the bulk silicon then

$$
\frac{d \lambda}{d T}=\left(\frac{1}{n} \frac{d n}{d T}+\alpha\right) \lambda
$$

The thermooptic coefficient $(\mathrm{dn} / \mathrm{dT})$ of the silicon is $1.86 \times 10^{-4} / \mathrm{K}[5]$ which is much larger than its expansion coefficient. Hence, the second term is negligible and a positive shift of $0.082 \mathrm{~nm} / \mathrm{deg}$ in the resonant wavelength due to the increase of temperature is expected.

\subsubsection{External Heater}

The heaters required to thermally tune the devices can be external or integrated on the device. Both type of heaters were employed in this work. External heaters are the most straightforward way for heating a sample. There exist various device support stages (like Peltier stage [79]) and different thermoeletrical heaters (like the Newport TEC used in this work) to achieve this end.

Although using external heating is convenient, it does not provide precise control. There is always doubt as to whether the temperature of the sample is exactly that shown on the support stage. Moreover, there does not exist the ability for local heating in specific regions. Despite these disadvantages for external heating, it is widely used for thermal tuning of SOI devices. For example, in thermal tuning of photonic crystals as refractometric optical sensors [80], planar photonic crystal 
Sh. Homampour - Masters Thesis

microcavities [79], interferometric photonic sensors and many others.

\subsubsection{Integrated Heater}

Employing integrated tuning components for example heaters on photonic devices, is an approach which leads towards fabrication of actively controlled devices. Integrated heaters have been used in thermooptic switches specifically those using a Mach-Zender geometry [81] and those requiring thermooptical tuning of SOI resonator swtiches [11].

One of the common methods of integration is to use resistive heaters. In such devices, heat is generated by application of current to a metal strip where resistance causes heat to dissipate into the guiding/device layer. To optically isolate the waveguides and protect the optical mode propagating in the device, an insulator layer usually oxide must be placed between the device layer and the metal. Common metals used as heaters are platinum, gold, nickel, chrome, aluminum, and tungsten. In this work aluminum was the metal of choice.

The amount of heat generated by aluminum strips on a device surface is calculated via equation 2.93

$$
Q=\frac{P}{V}=\frac{I^{2} \times R}{V}
$$

where $P$ is the applied power proportional to the applied current $(I)$ and the resistivity of the aluminum layer $(R)$, and $V$ is the volume of the aluminum layer. The resistivity of the aluminum is calculated by

$$
R=\frac{\text { Length }}{\text { Width } \times \text { Thickness }} \frac{1}{\sigma}
$$


Sh. Homampour - Masters Thesis

with $\sigma$ being the aluminum conductivity that is $3.69 \times 10^{9}\left(\mathrm{~S}^{-1}\right)$ [82].

The minimum thickness of oxide between the aluminum and the silicon waveguide such that no significant propagation loss was introduced was determined using the BPM method. In this way, a thickness of $300 \mathrm{~nm}$ was determined to be sufficient for preventing any optical loss due to the aluminum layer. The simulated corresponding mode and power to $200 \mathrm{~nm}$ and $300 \mathrm{~nm}$ thick oxide layer is illustrated in Figure 2.12

The heat transfer between the $\mathrm{Al}$ and Si layers must be calculated to determine values for the required aluminum thickness and applied voltage. In this system, heat is transferred by conduction and its rate is calculated by employing [83]

$$
\dot{Q}=-K A \frac{\Delta T}{\Delta X}
$$

where $K$ is the thermal conductivity, $\Delta \mathrm{X}$ is the layer's thickness, and $A$ is the surface area. The negative sign accounts for the different directions of heat flux and temperature gradient. 

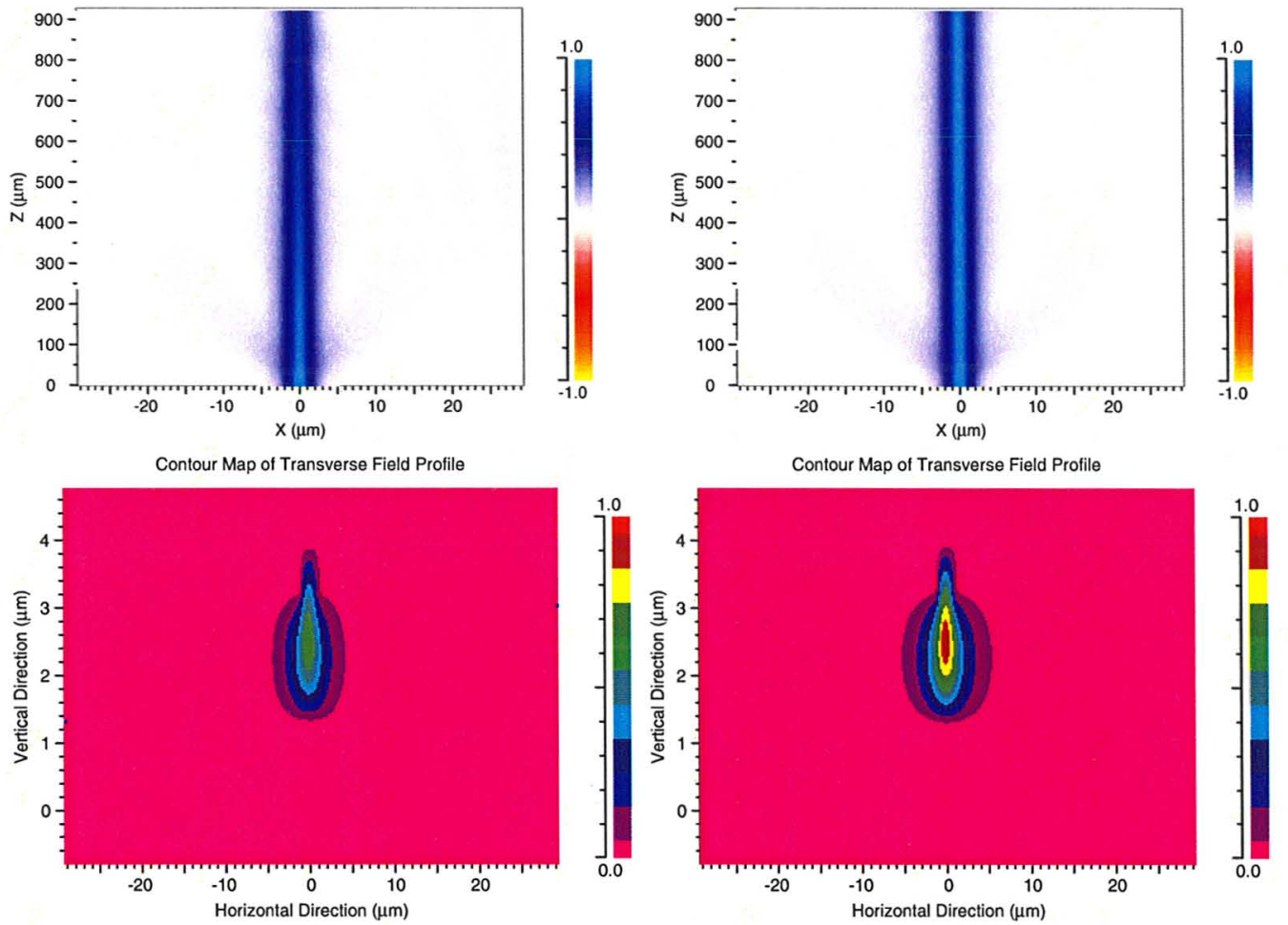

Figure 2.12: TE Mode profile and the corresponding contour map of the transverse field for $200 \mathrm{~nm}$ oxide layer on left and that of $300 \mathrm{~nm}$ oxide layer on the right

The heat transfer simulations were conducted using the heat transfer mode of the Comsol Multiphysics software, the resulting profile is shown in Figure 2.13. The simulations verified that a current of $200 \mathrm{~mA}$ passing through a $100 \mathrm{~nm}$ aluminum layer is sufficient to raise the waveguide's temperature more than 10 degrees. The fabrication and application of such a heater is discussed in more detail later. 

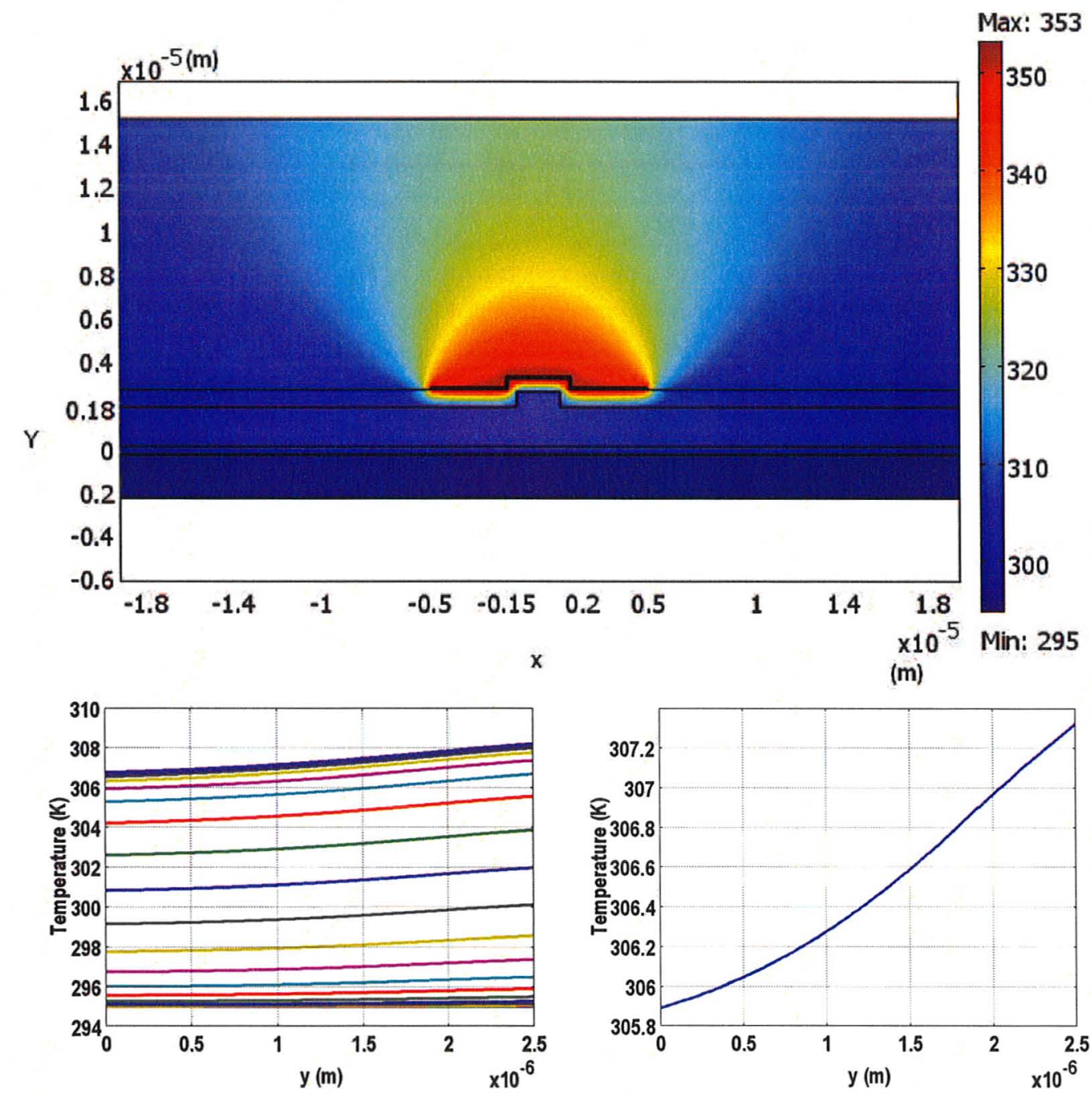

Figure 2.13: The heat transfer profile on top and the temperature change over time in the entire waveguide at the bottom left and the temperature difference between the top and bottom parts of the rib at the end at the bottom right 


\section{Chapter 3}

\section{Fabrication}

\subsection{Fabrication of Silicon Photonic Devices}

The majority of fabrication techniques for silicon photonic devices are adapted from those for III-V photonic devices and silicon microelectronics. This approach takes advantage of the existing vast knowledge of the fabrication processes and facilities used in silicon-based electronic device fabrication.

Silicon-On-Insulator (SOI) wafers are the platform and foundation of silicon photonic devices. These structures were originally conceived as method to reduce the parasitic capacitance in transistor circuits. Fabrication of these wafers and their characteristics play an important role in photonic device fabrication. There are three common methods used to manufacture SOI wafers with maximum customizability in the thicknesses of the silicon overlayer and buried oxide layer: (1) Separation by Oxide Implantation (SIMOX), (2) Bond and Etch back (BESOI), and (3) Smart-Cut [84].

The SIMOX process is the oldest approach and involves implantation of high 
Sh. Homampour - Masters Thesis

dose oxygen over the entire surface of the wafer to achieve buried layer of high oxygen concentration. The high temperature anneal of about $1300^{\circ} \mathrm{C}$ afterwards transforms this layer into high quality silicon dioxide. The implantation parameters and profiles define the depth, profile, and thickness of the oxide layer. Normally, this type of SOI wafer has a silicon overlayer of about 0.3 micron and an approximately 0.5 micron thick oxide layer [5]. If a thicker overlayer is required, it may be achieved via a post fabrication epitaxial growth.

In the BESOI method more physical manipulation is involved. First hydrophilic layers of silicon dioxide are grown on the surface of the two silicon wafers followed by formation of the chemical bonds that are achieved by bringing the wafers into intimate contact. Finally, the silicon overlayer is formed by back etching and thinning one of the wafers. This process does not produce SOI wafers with a high degree of control in thickness and uniformity due to the imprecise nature of the wafer thinning.

A process that combines aspects of SIMOX and BESOI is Smart Cut. In this approach one of the wafers is oxidized and then implanted with high energy $\mathrm{H}^{+}$ ions which weakens the silicon lattice along a particular plane prior to bonding the wafer to another silicon handling wafer. The subsequent thermal annealing causes the implanted wafer to split along the implant-damaged silicon plane. A fine chemical mechanical polish (CVP) is the last required step thereafter. This process offers flexibility and efficient use of silicon and results in high quality wafers from a material and uniformity perspective. Although wafers produced by BESOI process have the least uniformity, they may be made with a thick silicon overlayer which is damage free.

Another significant component and the most fundamental part of silicon pho- 
Sh. Homampour - Masters Thesis

Engineering Physics, McMaster University

Chapter 3. Fabrication

tonic devices is the rib waveguide. Its precise dimensions and fabrication process are crucial. To etch the waveguide to the required depth several methods are possible. The etch processes are divided into two categories: wet etch and dry etch. In wet etch, liquid chemical solutions are involved and based on the desired profile the chemistry may be varied. Potassium hydroxide $(\mathrm{KOH})$ and Tetramethylammonium hydroxide (TMAH) are known to be anisotropic etchants that result in a $54.7^{0}$ sloped side walls for SOI waveguide aligned in the $<100>$ direction [85].

On the other hand, in dry etch different combinations of gases, such as $\mathrm{SF}_{6} / \mathrm{O}_{2}$ [86], $\mathrm{CF}_{4}$ or $\mathrm{CH}_{3} \mathrm{Br}$ [36] are applied to the sample to etch the layers. Dry etch is very common for anisotropic RIE etch resulting in vertical sidewalls, but it is also flexible enough that tapered sidewalls are also attainable. Etch processes and etchers are discussed in much more detail in the following sections of this chapter.

Other standard CMOS processes including photolithography, metallization, and of course ion implantation are utilized in the fabrication of integrated silicon photonic devices.

\subsection{Fabrication of Bragg Gratings}

Following the introduction to silicon photonic devices and the procedures employed in their fabrication, it is now appropriate to explore the more challenging task of fabricating Bragg gratings. The required periodicity of Bragg gratings is on the order of $220 \mathrm{~nm}$ to $500 \mathrm{~nm}$ which places them beyond the reach of conventional photolithography available in the vast majority of academic fabrication facilities (including those at McMaster). Therefore, other fabrication methods have been developed to achieve the essential high spatial resolution. 
Sh. Homampour - Masters Thesis

\subsubsection{Lithographic Based Fabrication Process}

The use of photolithography to define features on the surface of a wafer is dominant in the microelectronic industry. Similarly, interference lithography is the pillar of the integrated Bragg grating fabrication. The basis of the interference technique is the spatial overlap of two coherent plane waves and formation of a standing wave which is recorded in photoresist or other photosensitive materials. The periodicity of the standing wave is computed through

$$
\Lambda=\frac{\lambda_{I L}}{2 \sin \theta}
$$

where $\lambda_{I L}$ is the illuminating wavelength and $\theta$ is the half angle between the two interfering beams. To achieve perfectly periodic standing waves over the entire sample the maintenance of spatial coherence of the standing wave is very important. The spatial coherence of the standing wave depends directly on the spatial and temporal coherence of the interfering beams. The standing wave created as the result of interference holography setup used in this work is illustrated in Figure 3.1

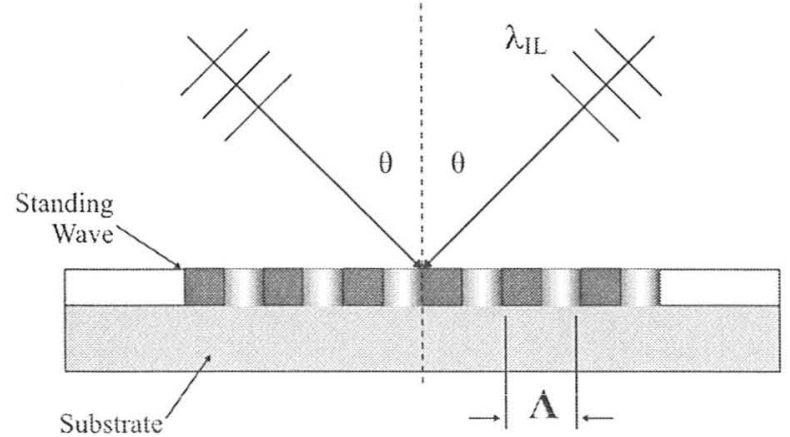

Figure 3.1: Standing waves produced by interference holography on a photosensitive material

The Bragg gratings developed in this work were manufactured using a variant of 
the Lloyd's mirror configuration. Lloyd's mirror is a classical setup of a two beam interference system. It consists of a laser source in this case a $325 \mathrm{~nm} \mathrm{HeCd}$ laser, a pinhole to spatially filter any higher order lasing mode (thus ensuring spatially uniform optical beam intensity) and a mirror mounted perpendicular to the sample holder. This mirror folds half of the beam back to the sample to create the interference pattern. Hence, the angle of the mirror with respect to the incident beam specifies the periodicity of the fringes and the pattern periodicity can be adjusted by angular rotation of the mirror. The optical setup is shown in Figure 3.2.

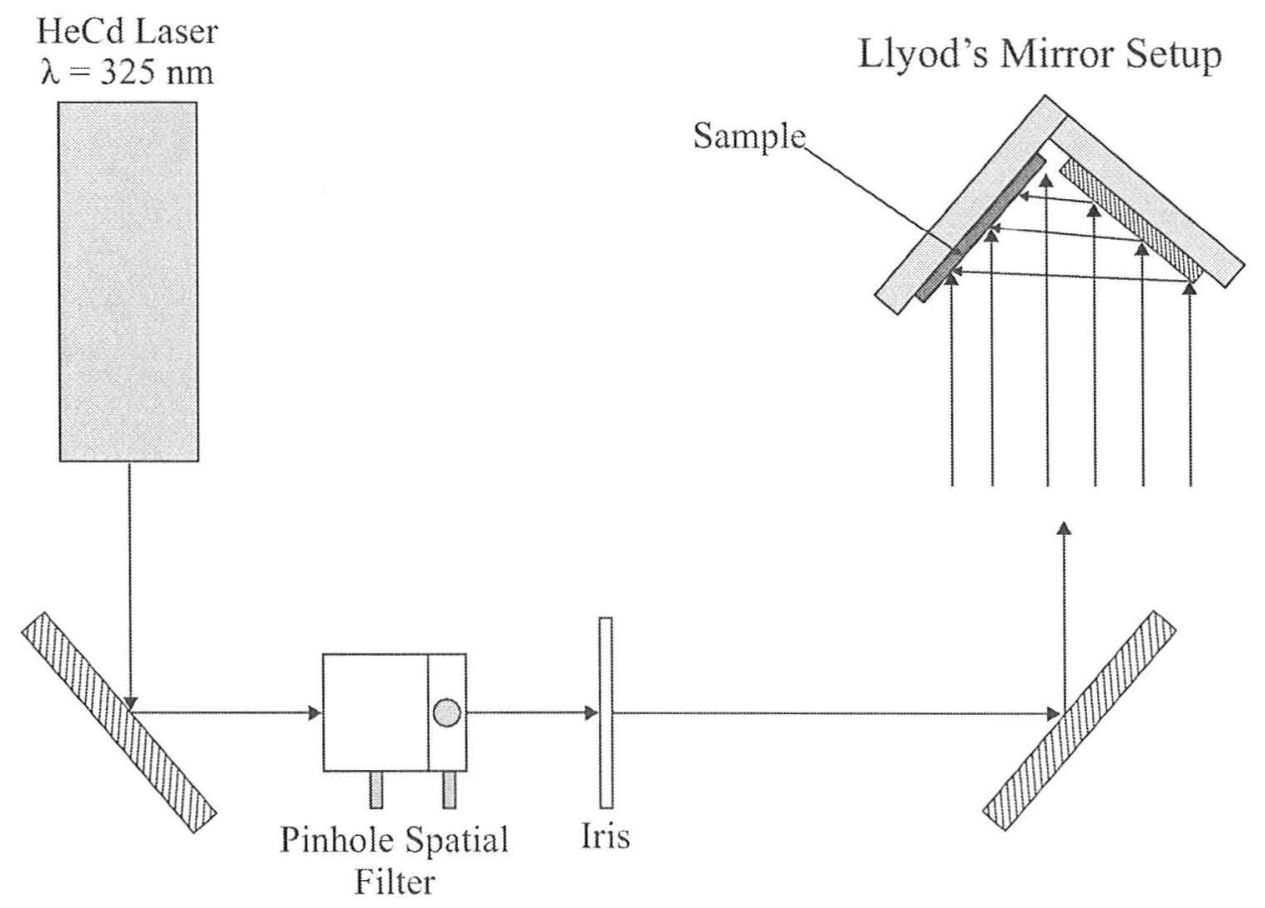

Figure 3.2: Lloyd's mirror setup

Ensuring planar waves at the sample is the most important step in using this setup since it defines the uniformity in the periodicity of the Bragg grating patterns. The waves leaving the light source have a Gaussian profile with non-uniform intensity, which results in a varying exposure and develop time over the surface of the 
sample. However, as the beam travels through space it expands and approaches planarity while its intensity is reduced. Thus, there is a trade off between the planarity of the beam and its intensity. Therefore, the optical path length plays a crucial role. The longer it is, the more uniform the beam will be over a greater area but at the same time the intensity will be lower which would result in impractical exposure times due to the specific exposing power required. Moreover, extremely careful adjustments of the pinhole and manipulation of the beam overlap are required to produce uniform exposure intensities.

Another issue with the interference lithography process is the ripples in the resist's edge which are detrimental to the line width control. The perpendicular standing wave produced due to the reflections from the surface of the wafer has a high aspect ratio with respect to the Bragg gratings leading to ripples at the edge. Moreover, to prevent further reflections from the substrate the thickness of the photoresist layer must be half the period of the perpendicular standing wave pattern, which is about $150 \mathrm{~nm}$. However, it is noted that antireflection coatings can also be applied to prevent this type of reflection.

Although Lloyd's setup is very versatile with high spatial resolution, there are important drawbacks that make its implementation in the more standard CMOS fabrication process difficult and not very desirable. One of the major drawbacks is the pattern inconsistency between consecutive samples which is due to the high sensitivity of the grating patterns to the optical alignment of the setup. Hence, it is very difficult to repeat exactly the same pattern on two samples. Another drawback is the inability to align fringes with existing patterns which leads to fabrication complexities. Furthermore, the interference effects caused by existing patterns on the substrate add to the disadvantages of employing such substrates. Therefore, 
Sh. Homampour - Masters Thesis

other lithography methods have been developed.

Employing X-rays and combining them with interference lithography is one of these approaches. First an X-ray mask is patterned using interference lithography, which can be outfitted with alignment marks, and then X-rays are applied to pattern the substrate. The mask makes the pattern repeatable while X-ray characteristics make the patterning much less complex. The X-ray wavelengths are absorbed by most of photoresist material, which eliminates the reflection problems. Further details can be found in [87].

Another alternative is electron beam lithography. This method is the most flexible with inherently high resolution. In this system a focused e-beam is traced over the surface of the sample and a prescribed pattern is defined. The ability of the system to narrowly focus the beam results in its very high resolution on the order of few nm's. Although this technique is very flexible, it has a low throughput and it is very time consuming since each pixel comprising the pattern must be exposed separately. Patterning a surface area requires an e-beam combined with an interferometrically controlled stage motion which imposes limitations on the accuracy of the pattern placements. Factors including thermal expansion, charging, beam current and focus drift conspire to make it more difficult than photolithography. Furthermore, the surface patterning in this system involves stitching small fields together, which leads to distortion and stitching errors that deteriorate the Bragg gratings coherence. The e-beam lithography is mostly used to fabricate prototype devices and generate masks that can be utilized in more conventional lithographic processes.

Another alternative approach to producing fringe patterns is the use of a phase mask. As light impinges normal to a phase mask, it diffracts into the first three 
Sh. Homampour - Masters Thesis

Engineering Physics, McMaster University

Chapter 3. Fabrication

orders. The $0^{\text {th }}$ order may be suppressed through the design of the mask and -1 and +1 diffraction orders interfere and produce a standing wave pattern. The mask results in the fringe pattern regardless of the illuminated wavelength hence, narrow wavelengths are not essential [88].

\subsubsection{Other Techniques for the Fabrication of Bragg Gratings}

Focused-Ion-Beam (FIB) milling is a fabrication technique that has gained a lot of popularity and is considered as an alternative to e-beam lithography. This technique is very flexible, ideal for fast prototyping, and can produce extremely controlled very fine features. It writes patterns directly on the sample surface and has been used for a range of applications including production of fine surface relief gratings in SOI substrates. In this technique energetic ions are incident on the sample and cause the sputtering of tiny amounts of material. The repetition of this process forms the pattern. However, high acceleration potentials required for focusing the beam causes ion implantation, crystal damage and amorphization in the processed material which leads to attenuation of light and lossy devices [28, 89, 90].

Nanoimprint lithography is a new lithography technique that can be used to fabricate Bragg gratings. The pattern is molded to the surface and the shape of the layer is changed instead of changing the chemical structure of the resist layer. Thus, PMMA polymer is used instead of photoresist. This technique has the significant advantage of a high throughput and low cost [91].

Deep-UV contact lithography is one of the other possible fabrication methods where a pre-existing mask pattern is brought into close contact with the substrate. For an appropriate design of the mask and resist, the UV illumination enables the near-field effects to transfer the pattern to the resist layer. Employing near optical 
Sh. Homampour - Masters Thesis

Engineering Physics, McMaster University

Chapter 3. Fabrication

fields, leads to feature resolutions beyond the diffraction limits [92]

\subsection{Implanted Bragg Gratings in SOI Waveguides}

All of the Bragg gratings described in this thesis, except for the broadband grating couplers discussed in section 3.6 and 4.3, were fabricated on SIMOX SOI wafers with a $2.5 \mu \mathrm{m}$ thick device layer, $0.375 \mu \mathrm{m}$ buried oxide layer, and $625 \mu \mathrm{m}$ base layer. The wafers were lightly p-doped (boron) to produce a nominal resistivity of $20-30 \Omega \mathrm{cm}$. The samples were initially cleaved into one inch squares and cleaned rigorously to remove any organic or metallic residue and the native oxide. The cleaning procedure consisted of twice repeated acetone, methanol, deionised water (DI) rinse followed by 10 minutes $\mathrm{H}_{2} \mathrm{SO}_{4}: \mathrm{H}_{2} \mathrm{O}_{2}$ (4:1) etch, 20 minutes of $\mathrm{UV}$ ozone growth and subsequent one and a half minute hydrofluoric (HF) etch. The outline of the fabrication process of ion implanted Bragg gratings in the SOI waveguide is illustrated in Figure 3.3.

\section{A) Deposition of the Buffer and the Implantation Mask Layer}

After the rigorous cleaning of the sample, a silicon dioxide layer with thickness of about $25 \mathrm{~nm}$ was deposited on the sample as a buffer layer. This layer serves as the etch stop layer when grating patterns are transferred to the a-Si layer by etching and the lift off layer when removing the a-Si layer. The second layer deposited was a $250 \mathrm{~nm}$ thick a-Si layer to be used as an implantation mask. The required thickness of this layer was determined using SSUPREM4 to ensure adequate stoppage of $\mathrm{Si}^{+}$ions while implanting with energies of up to $60 \mathrm{KeV}$. Both of the layers were deposited with an Inductively Couple Plasma Enhanced Chemical Vapour Deposi- 
Sh. Homampour - Masters Thesis

A)

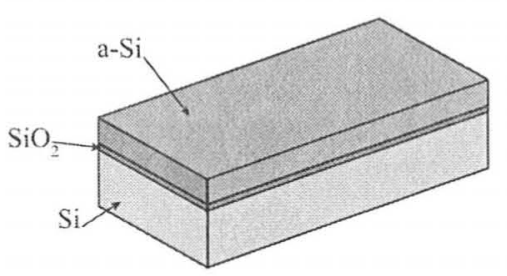

C)

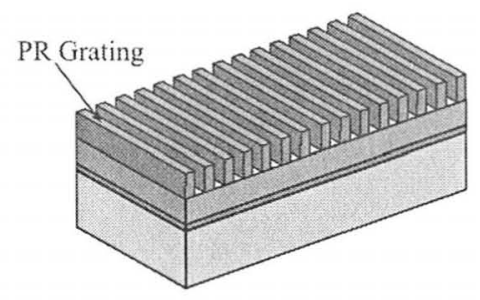

E)

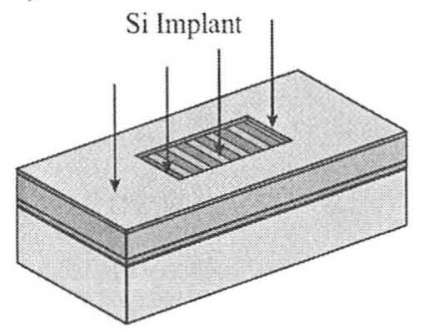

G)

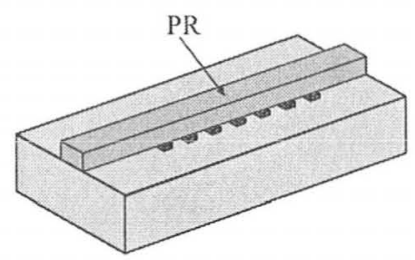

B)

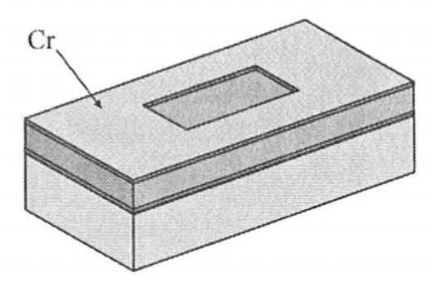

D)

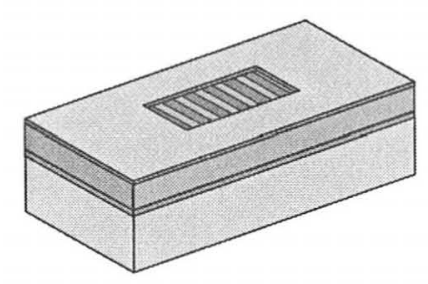

F)

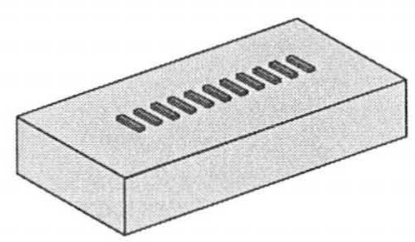

H)

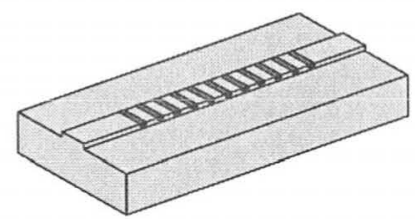

Figure 3.3: Fabrication process of implanted Bragg gratings on SOI waveguide 
Sh. Homampour - Masters Thesis

tion (ICP-PECVD) chamber at McMaster University.

Following the depositions, samples were annealed at $1000^{\circ} \mathrm{C}$ for 45 minutes in a nitrogen ambient under atmospheric pressure to rigidify the layers and reduce void concentrations. The annealing of the a-Si layer film crystallizes the layer and results in a poly-Si structure. The poly-Si layer is preferred over the a-Si layer since it has etching characteristics close to that for c-Si (etching conditions that are well known).

\section{B) Defining Grating Windows}

Since the operation conditions of the holography system do not allow one to define the placement of the gratings on specific areas of the surface, windows were employed to localize the gratings. Prior to patterning the Bragg gratings with the Lloyd's mirror setup, grating windows with the width of $100 \mu \mathrm{m}$ and varying length of 350-2100 $\mu \mathrm{m}$ were patterned using a chromium lift-off process.

The Shipley 1808 positive photoresist was spun at $4000 \mathrm{rpm}$ for 30 seconds to achieve a photoresist layer of about $800 \mathrm{~nm}$ thickness. The pre-spinning of a primer enhances the adhesion of the photoresist to the sample. Following a 5 minute wait and soft bake at $90^{\circ} \mathrm{C}$ for 2 minutes, the photoresist was exposed using $35 \mathrm{~mJ}$ from a Karl Suss MA6 mask aligner. To ensure that the gratings were perpendicular to the windows, the grating windows were aligned to the cleaved edge of the sample. The exposed sample was soaked in toluene without agitation for 6 minutes to harden the surface of the resist ensuring optimum lift-off results. After nitrogen blow drying, the sample was developed in a solution of Microposit 351 developer:DI (1:5) for 90 seconds. Using e-beam metalization, a $30 \mathrm{~nm}$ chromium layer was deposited on the sample to serve as a grating mask. It was lifted-off subsequently using an acetone 
Sh. Homampour - Masters Thesis

Engineering Physics, McMaster University

Chapter 3. Fabrication

bath. A 10-15 minute soak was sufficient for the lift-off process, however longer acetone bath or ultrasonic acetone for 2 minutes can be employed to remove any chromium residue without damaging the sample.

\section{C) Patterning the Holographic Photoresist Gratings}

Gratings with a period of $222 \mathrm{~nm}$ were defined using the Lloyd's mirror setup described in Figure 3.2. The fabrication of the gratings is highly sensitive to the setup's alignment, therefore, to achieve repeatability the process steps must be followed exactly and in a timely manner.

1 - Soft bake : Sample was heated on the hotplate at $80^{\circ} \mathrm{C}$ for at least 5 minutes to ensure no water remained in the sample surface.

2 - Apply the thinned resist :A solution of Shipley 1808 PR:Thinner (2:3) was applied. A 30 second wait is required after application before moving to the next step.

3 - Spin : The resist was spun at 5000 rpm for 30 seconds.

\section{4 - Wait for 5 minutes}

5 - Soft bake : Sample was heated on a hotplate at $80^{\circ} \mathrm{C}$ for 1 minute to evaporate any remaining solvent in the resist and improve adhesion. A soft bake at this level also relieves the stresses due to spinning through thermal relaxation.

6 - Wait for 30 seconds : After the soft bake wait for 30 seconds and then transfer the sample to the holography station.

7 - Exposure : The sample was exposed to $25 \mathrm{~mJ}$ of energy.

8 - Develop : The sample was developed in Shipley CD-30 while agitating slowly for approximately 45 seconds.

9 - Rinse : The sample was rinsed in DI water for 30 seconds.

10 - Blow dry : Blow dry with $\mathrm{N}_{2}$ carefully. 
11 - Hard Bake : The sample was hard baked on the hotplate at $110^{\circ} \mathrm{C}$ for 2 minutes to harden the resist.

It is noted that to achieve a good line width control (lateral feature size of 100 $\mathrm{nm}$ ) a $100 \mathrm{~nm}$ thick photoresist (PR) layer is required therefore, thinned PR with the specified spin speed and time was used. Thinned PR requires a lower baking temperature and is more susceptible to variation in that temperature. If too hot, the process leads to patchy gratings and incomplete PR removal while too much of the resist is removed if the bake is too cold.

During exposure the sample must be placed such that the edge used in the alignment of the grating windows is at the bottom part of the sample holder to ensure the gratings are perpendicular to the grating windows. Although the exposure time can be calculated based on the energy received at the sample holder, the best method is to adjust the exposure time such that the time for resist to develop is approximately 45 seconds, conceding that the development is the most difficult step to control.

The developing step is very important since a few seconds less or more than that required leads to under or over development. Therefore, close color inspection of the surface is crucial while developing, coupled with agitating slowly. When the sample is submerged in the solution, it turns to a dark blue color and remains in this state for most of the developing stage. After about 30 seconds the sample begins to turn into a beige color. The key to the development process is to rapidly remove the sample as soon as the entire surface of the sample has the uniform beige color and immerse it in DI water. The best gratings are achieved when the total development time is about 45 seconds.

Diffraction from a white light source can be used to test the integrity and uniformity of the grating patterns. Under the proper angle the diffracted light should be a 
Sh. Homampour - Masters Thesis

Engineering Physics, McMaster University

Chapter 3. Fabrication

uniform pale blue. The presence of black regions on the surface signifies underdevelopment while shiny regions suggest overdevelopment. Inspection of the gratings using an SEM allows confirmation of a continuous gratings across the chromium layer and signifies that the presence of these chromium windows has little effect on the spinning and development of the grating patterns.

\section{D) Etching the PR Gratings into the poly-Si Mask}

To realize a suitable ion implantation hard mask, poly-Si layer was used. Thus, the grating pattern had to be transferred from the PR to the poly-Si layer. The Reactive Ion Etch (RIE) was accomplished using a Bosch process on an Alcatel 601E RIE etcher at University of Western Ontarion (UWO). The Bosch Process in this system cycles between an etch step with sulfur hexafluoride $\left(\mathrm{SF}_{6}\right)$ for 2 seconds and a passivation cycle with Octafluorocyclobutane $\left(\mathrm{C}_{4} \mathrm{~F}_{8}\right)$ for 1 second to gain vertical sidewalls with aspect ratio of about 20:1. The etches were all performed under pressure of $1 \times 10^{-2} \mathrm{mBar}$, plasma source $\mathrm{RF}$ power of $1000 \mathrm{~W}$, substrate bias $\mathrm{RF}$ power of $80 \mathrm{~W}$, with $\mathrm{He}$ cooled backside at $20^{\circ} \mathrm{C}$.

The etch rate of photoresist, poly-Si, silicon dioxide, and chromium were found to be $\sim 100 \mathrm{~nm} / \mathrm{min}, \sim 275 \mathrm{~nm} / \mathrm{min}, \sim 25 \mathrm{~nm} / \mathrm{min}$, and $<5 \mathrm{~nm} / \mathrm{min}$ respectively. Hence, there is a selectivity of $2.75: 1$ of poly-Si to PR which makes the $100 \mathrm{~nm}$ PR gratings sufficient for etching the poly-Si hard mask. The etching process was carried out for 1 minute without any concern for over etching due to the existence of the silicon dioxide layer which acts as the buffer and etch stop layer. Furthermore, the large selectivity between the chromium and poly-Si ensured that only gratings at the grating windows would be etched in the poly-Si layer. Etched gratings in poly-Si and the effect of the chromium window are shown in the SEM image in 


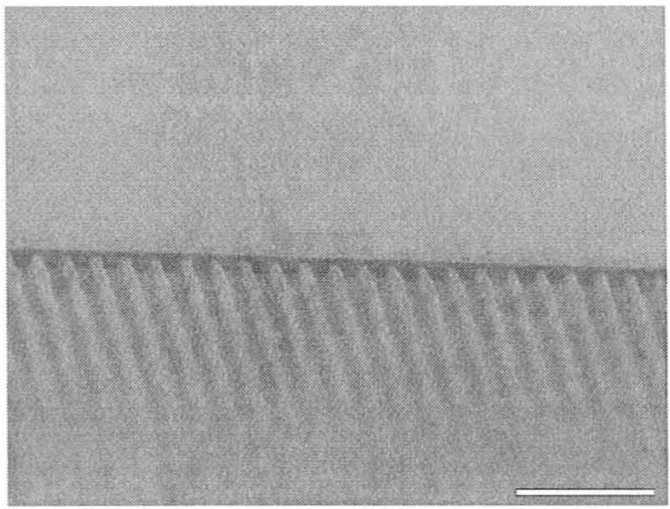

Figure 3.4: Etched PR grating and the effect of the chromium grating windows. The length bar indicates $1 \mu \mathrm{m}$. The patterns on the surface are due to the gold coating for better SEM resolution.

Figure 3.4. The PR residuals after RIE were removed by 10 minutes $\mathrm{O}_{2}$ plasma ash. Again at this point the white light test can be performed and a uniform light blue diffraction can be seen at the grating sections signifying a good transfer of the grating patterns into the poly-Si.

\section{E) $\mathrm{Si}^{+}$Ion Implantation}

In this step silicon ions were implanted to produce a damaged induced amorphous silicon layer. The implantation was done using the ion implanter at UWO. The samples were implanted at the nominal dose of $2 \times 10^{15} \mathrm{ion} / \mathrm{cm}^{2}$ at the liquid nitrogen temperature of $77 \mathrm{~K}$ with 50 and $60 \mathrm{KeV}$. During implantation a sample tilt of $0^{\circ}$ was used since the presence of silicon dioxide minimizes the channeling due to its non-crystalline structure. Good thermal contact between the sample and sample holder during the process is crucial to prevent possible thermal annealing.

\section{F) Removal of Masking Layers}

Prior to patterning the ribs on the implanted Bragg gratings, the implantation 
mask must be removed. The first step was to remove the chromium grating window via a selective chromium etch in Cyantek's CR-100 etchant.

The next step was to remove the poly-Si and oxide layers. The sample was submersed in buffered HF (BHF) for about 17 minutes. The sample must be left in the HF until both of the layers are completely removed and the shiny surface of the silicon is visible. The submersing of the sample in BHF results in two effects. One is the etching of the oxygen contents in the PECVD grown poly-Si layer and second is the seeping of BHF through grating teeth into the silicon dioxide layer which leads into lift-off of the poly-Si layer. As the BHF seeps into the oxide layer through the voids present in poly-Si layer, it interacts with and etches away the oxide layer thus, a quick and easy lift-off happens.

After the mask removal a visible color difference exists in the ion implanted regions on the surface of the SOI, which indicates the amorphization of the silicon in those areas as the result of the damage introduced by ion implantation.

\section{G) Rib Patterning}

The rib structures were patterned on the planar surface of the implanted SOI using standard photolithography techniques. Shipley 1808 was spun on the surface (after deposition of the primer) at $4000 \mathrm{rpm}$ for 30 seconds and then soft baked for 2 minutes at $110^{\circ}$. Then rib patterns were defined on the $800 \mathrm{~nm}$ thick photoresist layer using Karl Suss MA6 mask aligner, developed in a solution of Microposit 351 developer:DI (1:5) for about 30 seconds and finally, hard baked at $130^{\circ} \mathrm{C}$ for 2 minutes. These PR ribs were used as the etching mask in the RIE etching of the rib waveguides. 
Sh. Homampour - Masters Thesis

Engineering Physics, McMaster University

Chapter 3. Fabrication

\section{H) Etching the rib and Preparing the Facets}

The rib waveguides were etched into the SOI sample using Alcatel 601E RIE etcher at UWO. The etching rate of the silicon was determined to be $1 \mu \mathrm{m} / \mathrm{min}$, making the selectivity of the PR to silicon 20:1.The etch depth of the ribs was targeted to be $700 \mathrm{~nm}$, hence the thickness of the PR mask was deemed adequate.

Optical facets were prepared through a thinning and cleaving process, where the samples are thinned to a thickness of 220 to $250 \mu \mathrm{m}$ for producing optimal results. The samples were mounted on a sanding block facedown using crystal bond and then backside-thinned with wet silicon carbide paper. After thinning, the mounting blocks were heated to $150^{\circ} \mathrm{C}$ to remove the sample. Any residual crystal bond was removed in an acetone bath. A SEM picture of the completed ion implanted SOI rib waveguide is illustrated in Figure 3.5.

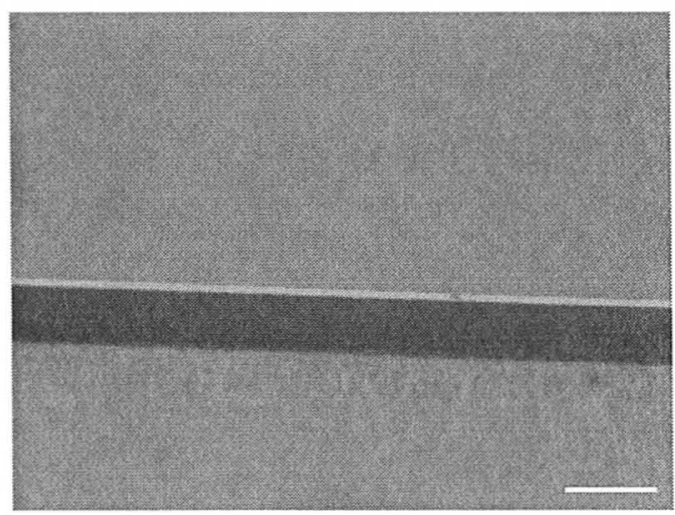

Figure 3.5: A completed ion implanted rib waveguide with a planar surface. The length bar indicates $1 \mu \mathrm{m}$.

The resulting samples are ready to be heated by external heater and be measured however, to fabricate the integrated heaters the last step should be modified and more steps must be added as will be discussed in the next sections. 


\subsection{Etched Gratings in SOI Waveguides}

It is interesting to compare the ion implanted Bragg gratings on SOI waveguides with more traditional, etched Bragg gratings. Etched gratings were fabricated using a technique similar to that described above. In fact the process of etched grating is less complex. The buffer and mask layers are not required since there is no implantation involved thus, step A is skipped. After patterning the PR gratings, they were etched to a depth of $120 \mathrm{~nm}$. The removal of the chromium layer was done with the same process and finally, ribs were patterned and etched. Since the gratings are etched they are visible at all times. A SEM picture of such gratings is shown in the Figure 3.6

Figure 3.6: Etched grating on SOI rib waveguide. The length bar represents $100 \mathrm{~nm}$. 
Sh. Homampour - Masters Thesis

Engineering Physics, McMaster University

Chapter 3. Fabrication

\subsection{Integrated Heaters on Implanted Bragg}

\section{Gratings in SOI Waveguides}

To prepare the integrated heaters on either the samples with implanted Bragg grating or those with etched gratings the last step in section 3.3 must be modified. The ribs may be etched but the facets should not yet be prepared. The outline of the steps is illustrated in Figure 3.7

I)

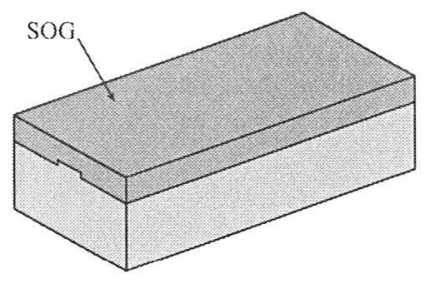

K)

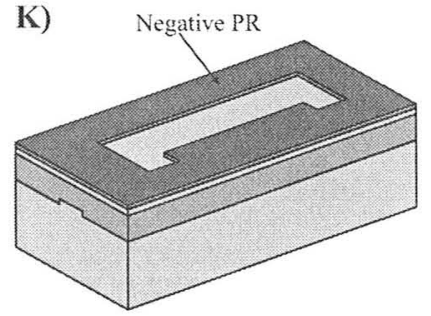

J)

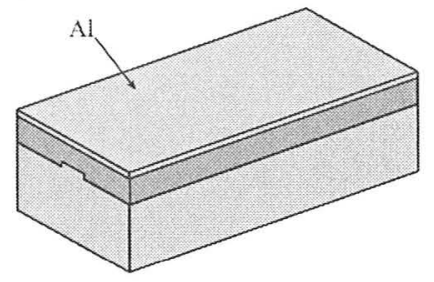

L)

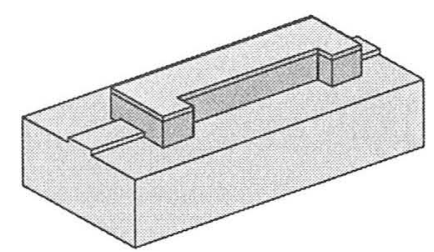

Figure 3.7: Fabrication process for integrating heaters

\section{I) Deposition of Spin-On-Glass on the Ribs}

After etching the ribs, an oxide layer is deposited to prevent any optical propagation loss due to the aluminum layer covering the waveguides. However, a silicon dioxide layer prepared via growth or deposition cannot be used due to the temperatures involved in depositing oxide layers or growing oxide thermally, which are 
above $200^{\circ} \mathrm{C}$ and will remove the damage induced by the implanted ions. Therefore, spin-on-glass (SOG) was employed instead. The SOG remains in a quasi-solid state thus, solvents like acetone can still dissolve it. The Futurrex DC4-500 spinon-glass was spun on the sample at $3000 \mathrm{rpm}$ for 40 seconds following a 1 minute wait after applying SOG on the sample. Then the sample was heated on a hotplate at $150^{\circ}$ for 2 minutes. After waiting for 2 minutes the process was repeated 2 more times. The resulting SOG layer has a thickness of about $600 \mathrm{~nm}$ on the slab sides and $400 \mathrm{~nm}$ on the sidewalls and edges. The SEM image of this layer is shown in the Figure 3.8

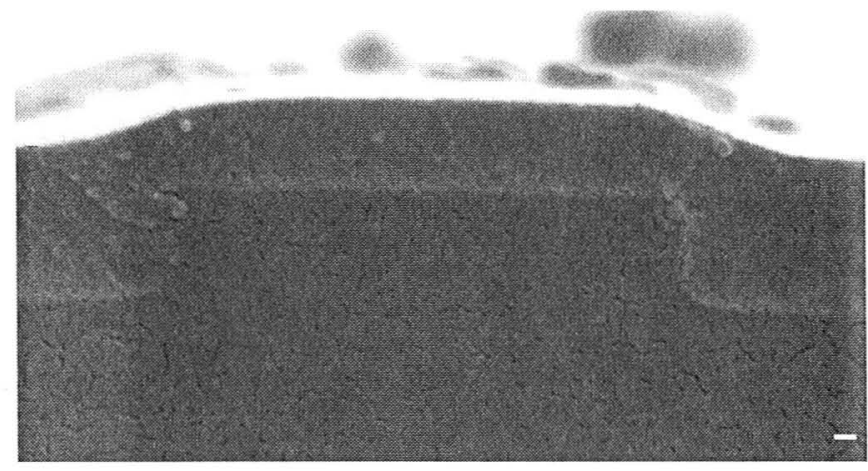

Figure 3.8: SEM image of SOG on the rib. The length bar indicates $100 \mathrm{~nm}$.

\section{J) Deposition of Aluminum Layer and Patterning the Aluminum Strips and}

\section{Contacts}

A $100 \mathrm{~nm}$ Aluminum layer was deposited using a bell jar setup at McMaster University and patterned using negative resist.

\section{K) Patterning the Aluminum Strips and Contacts}

The negative resist NR9-1500P was spun at $5000 \mathrm{rpm}$ for 40 seconds which 
Sh. Homampour - Masters Thesis

Engineering Physics, McMaster University

Chapter 3. Fabrication

resulted in a photoresist layer of about $1.2 \mu \mathrm{m}$ thick. The sample was soft baked at $150^{\circ} \mathrm{C}$ for 60 seconds and then exposed with $30 \mathrm{~mJ}$ of energy. Following the soft bake at $100^{\circ} \mathrm{C}$ for 60 seconds, the sample was developed in RD6 for about 8 seconds, and rinsed in DI water.

\section{L) Etching the Aluminum Strips and Contacts}

To etch the patterns into Aluminum , the aluminum etchant by Transene company was heated up to $50^{\circ} \mathrm{C}$ which led to aluminum etch rate of $100 \AA$ /second thus, the etch took about 10 seconds. The sample was rinsed in DI water afterwards.

\section{M) Removing the Photoresist Residue and Preparing the Facets}

The photoresist residue was removed via $\mathrm{O}_{2}$ plasma ash for 4 minutes. The sample could not be cleaned by acetone since this would remove the SOG. Moreover, plasma ashing the samples longer than 4 minutes also started to attack the aluminum.

A Microace 3 dicing saw was used to dice the samples which were subsequently polished at the University of Surrey to smooth the optical facets. The thinning and cleaving technique could not be used due to the effect of acetone on SOG.

\subsection{Fabrication of Broadband Grating Coupler on SOI}

These samples were fabricated on a BESOI SOI wafers with $5 \mu \mathrm{m}$ thick device layer, $1 \mu \mathrm{m}$ buried oxide layer, and $625 \mu \mathrm{m}$ base layer. The wafers were lightly pdoped (boron) to produce a nominal resistivity of $20-30 \Omega \mathrm{cm}$. The samples were 
Sh. Homampour - Masters Thesis

Engineering Physics, McMaster University

Chapter 3. Fabrication

cleaved in one inch squares and rigorously cleaned in the same way described in section 3.3. Bragg gratings with period of $500 \mu \mathrm{m}$ were patterned using the Lloyd's mirror setup. The sample was placed in the setup so that only half of the sample would be exposed to the gratings. Thus, the sample consisted of two sections one with the grating and one "shiny side" without any grating. The gratings were etched using the STS 320PC parallel plate etcher at McMaster University to the depth of $120 \mathrm{~nm}$. The facets of these samples were prepared parallel to the gratings by thinning and cleaving as explained in part $\mathrm{H}$ of section 3.3.

\subsection{Development and Application of Fabrication}

\section{Processes}

The fabrication of silicon-based microstructures, including both photonic devices and CMOS devices, requires the ability to etch material from the surface of a wafer with extremely tight control over the vertical etch depth and the lateral profile. Etching processes can be broadly separated into two categories: wet etch and dry etch. After a very brief description of wet etching, we discuss below the dry etching processes that were developed in-house for the device fabrication reported in this thesis.

\subsubsection{Wet Etch}

Wet etching was originally the dominant etch process used in the microelectronics industry. Its low cost, reliability and high selectivity made it popular for defining patterns with feature sizes greater than $3 \mu \mathrm{m}$ [93].

In wet etching, the sample is usually immersed in a liquid etchant solution. 
Sh. Homampour - Masters Thesis

Engineering Physics, McMaster University

Chapter 3. Fabrication

Chemical reactions, which are the basis of this process, can be very selective; i.e. different materials etch at very different rates. The etchant reacts with the film to form a water-soluble byproduct or gases that diffuse away from the surface.

Temperature, composition, density of the film, and in some cases the crystallographic orientation ${ }^{1}$ all have a great effect on the etch rate. Potassium hydroxide ( $\mathrm{KOH})$, sodium hydroxide $(\mathrm{NaOH})$, and ammonium hydroxide $\left(\mathrm{NH}_{4} \mathrm{OH}\right)$ are some of the common etchants used for silicon wet etching $[2,93]$.

\subsubsection{Dry Etch}

In plasma or dry etching there are two dominant processes: chemical and physical. An etch can proceed by chemical reactions, physical sputtering or a combination of the two. This makes the process more complicated but it offers better resolution than wet etching. Dry etching has replaced wet etching in integrated circuit manufacturing in recent years for two main reasons. First, the etching can be more vigorous due to the very reactive chemical species produced in the chemical component of the etch. Second, and more important, etching can be highly directional since the physical etch processes are anisotropic. Thus, etching films with thicknesses comparable to the minimum pattern dimensions has become possible. In this process the sample is placed in a vacuum chamber into which the required gas(es) are admitted. The danger of direct exposure to and the handling and disposing of large amounts of harmful gases, acids and chemicals are substantially reduced $[2,93]$.

\footnotetext{
${ }^{1}$ The etch rates for two crystallographic directions in silicon using $\mathrm{KOH}$ are different [2]
} 


\subsubsection{Chemical Etching}

This process is based on the chemical reactions between the species that are present. Reactive species or free radicals are formed in the plasma and produce volatile etch byproducts by reacting with the substrate. Desorption of these products from the surface is the last step to complete the etch cycle. Free radicals are the product of the reaction between the free electrons in the plasma and the atoms of the desired material. They are electrically neutral with incomplete bonding and unpaired electrons. Thus, they are highly reactive and react with the material to be etched. The volatile nature of the byproducts causes more substrate to be exposed and etched. Gas additives are usually used to react with dissociated ${ }^{2}$ species and prevent their recombination. This increases the density of free radicals and etch rate. Chemical etching is very selective with low surface damage since it is based on chemical reactions. The isotropic distribution of arrival angles of the free radicals at the substrate surface and their low sticking coefficient makes this etch process isotropic [2, 3].

\subsubsection{Physical Etching}

In this process energetic ions accelerated by electric fields to an energy of more than $200 \mathrm{eV}$ are the dominant etch component. The energy and momentum of these ions are transferred to the substrate as they strike the atoms on the surface and sputter away the surface material. Normally, sputtering efficiency is described by sputtering yield, which is defined as the ratio of the number of atoms or molecules ejected from the target to the number of the incident ions. This is a function of the angle of incidence of the ions. The efficiency can be enhanced by considering the

\footnotetext{
${ }^{2}$ Some of the typical reactions occur in the plasma are dissociation, ionization, excitation, dissociation ionization, and recombination [2]
} 
Sh. Homampour - Masters Thesis

Engineering Physics, McMaster University

Chapter 3. Fabrication

chemistry involved in the sputtering, since ions have the potential to react with the surface atoms and dislodge more atoms.

The strongly directional nature of the ions makes the etch very anisotropic with significant surface damage, roughness and reduced selectivity. Moreover, the high sticking coefficient of the ions prevents them from striking the surface more than once, which leads to a more anisotropic etch. However, redeposition and trenching of the ejected species can occur due to their nonvolatile nature, which makes the process unfavorable in VLSI fabrication [2, 3, 93].

This method is slow compared to the other dry etch methods. The etch rate is several hundred angstroms per minute while in chemical etching it can be as high as $6 \mu \mathrm{m}$ per minute. Some of the plasma damage produced in the process can be removed by thermal annealing.

\subsubsection{Ion-Enhanced Etching}

Combining the purely chemical etch process with the sputter-based physical etch can overcome the shortcomings of both and enhance the etch results. This combination is called an Ion-Enhanced etch process and offers both the control of anisotropy and adequate selectivity in the etch with much higher etch rate. This process is not the linear combination of the two process and both components are required at each step for the process to work. The etched profile in this process is more similar to that of the physical etch but increasing the chemical component doesn't have the same effect as it would in the pure chemical process.

In this process the ion bombardment, in addition to dislodging surface material, enhances one of the chemical etch steps, such as etch reaction and formation of byproducts or removal of the byproducts. Moreover, some of the byproducts 
Sh. Homampour - Masters Thesis

Engineering Physics, McMaster University

Chapter 3. Fabrication

or other species from the plasma can be deposited on the surface to enhance the directionality of the etch. By changing the etch chemistry and plasma conditions different etch profiles with different slopes can be achieved, which makes the process more desirable $[2,93]$.

\subsubsection{Different Dry Etch Systems}

Different dry etch techniques and models have been developed based on the material being etched, the required chemistry in the plasma, and the profile specifications. These etchers can be categorized as Barrel Etchers, Parallel Plate Etchers, or High-Density Plasma Etchers. Considering ion enhancements can subdivide them into etchers in plasma mode or Ion-Enhanced mode. Some of the common plasma sources are Electron Cyclotron Resonance (ECR), Inductively Coupled Plasma (ICP), and Parallel Plate Systems [2, 3].

\section{Parallel Plate systems}

The parallel plate systems are among the more common ones used for plasma etching of thin films. These systems consist of two parallel electrodes in a vacuum chamber with an RF power source $(13.56 \mathrm{MHz})$ connected to one of the electrodes. The sample is placed on the bottom electrode. Ion directionality and density in such systems is low compared to the other systems, which leads to a lower etch rate and reduced selectivity. Thus, using a lower pressure and greater ion bombardment is recommended as will be explained in more detail below.

Plasma etching in parallel plate systems can be operated in two modes. The plasma mode in which chemical etching is the dominant process and the physical etching component can be neglected; and the reactive ion etching (RIE) mode in 
Sh. Homampour - Masters Thesis

Engineering Physics, McMaster University

Chapter 3. Fabrication

which the physical component of the etch is enhanced. The etcher used in McMaster falls into this latter category.

In reactive ion etching both chemical and physical mechanisms play an important role. In this mode, ion bombardment is stronger, ions have more energy, which enhances the physical component of the etching. This can be achieved by having a greater voltage drop between the plasma and the electrode or having a physically smaller lower electrode and grounding the upper electrode. The operating steps in an RIE etcher can be summarized as follows:

\section{Application of RF (radio frequency) signal to electrodes}

This step creates a high electric field across the electrodes in the vacuum chamber that ionizes some of the gas atoms and produces a plasma containing positive ions and free electrons. The gas pressure is usually stabilized between $10^{-3}$ and $10^{-2}$ Torr. Electrons traveling in the electric field between the electrodes gain enough energy (greater than ionization energy of atoms) to ionize the atoms by inelastic collisions. Collisions of less energetic electrons with atoms will only excite the atoms, which then will lose their energy by luminescing and a distinctive glow is observed. Therefore, once a plasma state is formed and an equilibrium between positively and negatively charged particles is reached, the collisions resulting in luminescence dominate over ionization collisions. The chamber pressure and electrode spacing are the two important parameters to initiate a discharge.

The greater mass of the ions, and the higher speed of the electrons compared to the ions, leads to loss of more electrons than ions to the electrodes and the creation of a positively biased plasma. This charge distribution determines the DC potential in the plasma which accelerates the positive ions towards the ground electrode 
Sh. Homampour - Masters Thesis

Engineering Physics, McMaster University

Chapter 3. Fabrication

where sample sits $[5,93]$.

\section{Creation of free radicals}

When electrons collide with gas atoms free radicals, which are electrically neutral but have incomplete bonding, are produced. These elements have unpaired electrons and therefore, they are highly reactive. For instance, considering $\mathrm{CF}_{4}$ the reaction becomes

$$
C F_{4}+e \rightarrow C F_{3}+F+e
$$

These components react with the material being etched, for instance silicon, and produce a byproduct that is volatile and leaves the surface. Hence, more silicon is exposed and etched.

$$
4 \mathrm{~F}+\mathrm{Si} \rightarrow \mathrm{SiF}_{4}
$$

Adding other gases can increase the density of free radicals by preventing recombination among them. For example, $\mathrm{O}_{2}$ atoms react with $\mathrm{CF}_{3}$ and $\mathrm{CF}_{2}$ and prevent them from recombining with $\mathrm{F}$. These reactive chemical components enhance chemical etching and increase the etch rate. They have a wide arrival angle distribution and enhance isotropic etching.

\section{Ionic components}

These contribute to the physical part of the etching. The electric field across the electrodes accelerates positive ions towards the electrodes almost normally and therefore, normal to the surface of the wafer. Ions strike the surface only once and their flux is more directional than that of the free radicals due to the directional electric field. Hence, the physical etching is more directional and anisotropic. Ionic 
Sh. Homampour - Masters Thesis

Engineering Physics, McMaster University

Chapter 3. Fabrication

components strike the atoms on the surface of the material and physically dislodge them. The energy of the ions must be greater than the surface binding energy of the atoms which is few hundred $\mathrm{eV}[5]$.

\section{Ion enhanced etching}

As the ions bombard the substrate, they enhance the etch process by:

-Breaking the atomic bonds, which enhances the chemical reactions between free radicals and surface of the material.

- Accelerating the formation and sputtering away the volatile byproducts.

- Removing the inhibitors, which are the residues that either block the chemical etch species or react with them. This improves the anisotropy of the etch.

The directionality of the ion flux has a major influence on the sidewall's profile. If the arrival angle of the ions is not normal to the surface, non-vertical sidewalls are obtained $[2,93]$.

\section{Barrel Etchers}

This type of etcher is one of the earliest etch systems developed for dry etching. The electrodes are wrapped around a quartz tube. Hence, they are curved. RF power is applied to the electrodes and a plasma is generated. The sample is placed vertically in the middle of the chamber and is shielded by a perforated metal shield to reduce the substrate exposure to charged, highly energetic species in plasma. Considering the design of the etcher, there cannot be any ion bombardment and the etch process is completely chemical. Therefore, the etch is very selective and isotropic but very slow and nonuniform. Normally, a barrel etcher is only used to remove photoresist residue due to the characteristics of its etch profile. Moreover, the etch or 
Sh. Homampour - Masters Thesis

Engineering Physics, McMaster University

Chapter 3. Fabrication

photoresist ashing is usually done over a long time to overcome the nonuniformity, which won't harm the sample due to the high selectivity of the process $[2,94]$. A schematic of such an etcher is shown in the Figure 3.9.
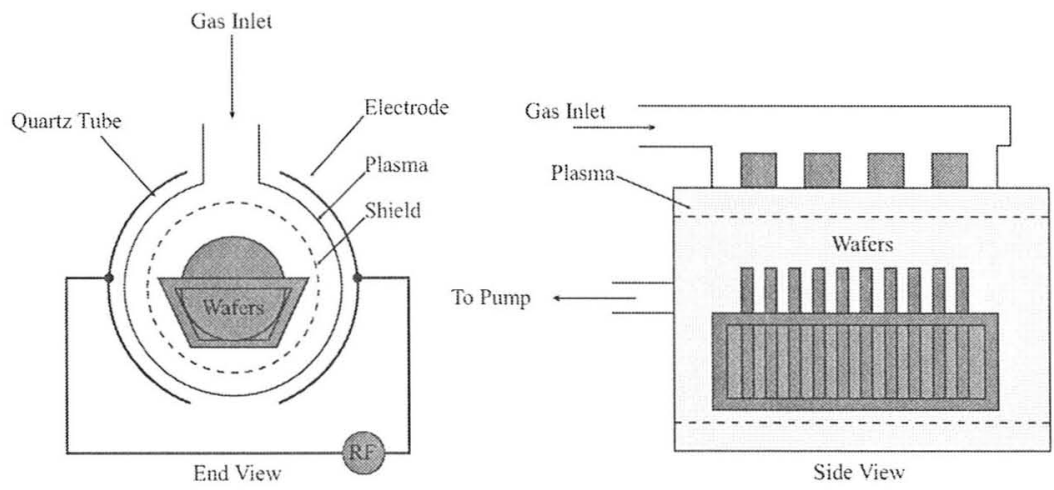

Figure 3.9: Barrel Etcher system [2]

\section{Other Ion-Enhanced Dry Etchers}

Inductively Coupled Plasma (ICP) systems represent the more recent developments in etching technology. The plasma densities in ICP systems are two to four orders of magnitude higher than in RIE systems.

The plasma is formed in a dielectric vessel encircled by an inductive coil and RF power is applied into the coil. These coils being in the horizontal plane, lead to an induced magnetic field in the vertical direction. This strong magnetic field traps the electrons in the center of the chamber and produces a high-density plasma. The plasma diffuses from the center to the substrate at low pressures with low ion energy [3].

The high density of the plasma makes utilizing lower pressures possible while having high ion flux and etch rate. A lower pressure not only facilitates more anisotropic and direct etch but also reduces the substrate damage that results from 
Sh. Homampour - Masters Thesis

Engineering Physics, McMaster University

Chapter 3. Fabrication

high sheath bias and ion energy. Also superimposing an RF bias on the sample increases the anisotropy of the etch and two power sources enable the system to control the energy of bombarding ions independently of the plasma density. The ICP system is popular due to its simpler design, easy wafer diameter scale-up, and lower cost and power requirements $[2,95]$. The schematic diagram of an ICP system is shown in Figures 3.10.

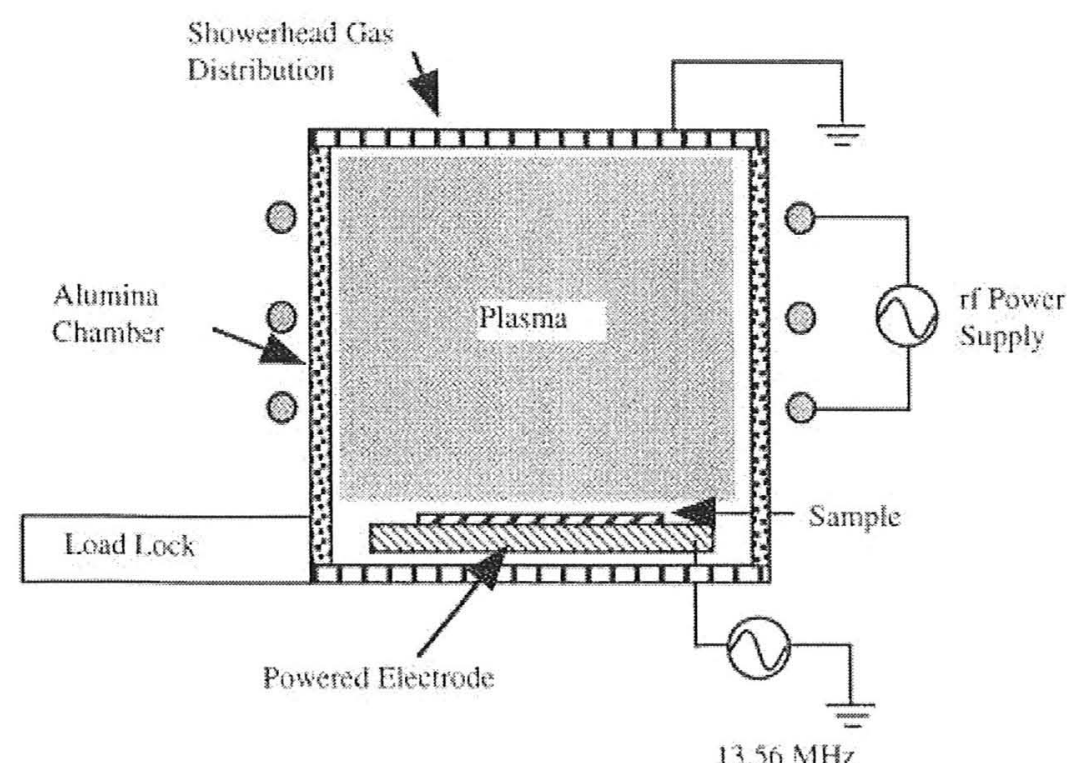

Figure 3.10: ICP Dry Etch system [3]

\subsubsection{The Important Parameters in an Etch Process}

\subsubsection{Bosch Process}

In the fabrication of optoelectronic and microelectromechanical systems (MEMS) using an anisotropic plasma for etching is indispensible and the Bosch process is one of the etch processes established to enhance the anisotropic characteristic of 
the etch resulting in vertical sidewalls, high etch rate, and high aspect ratio. This process consists of two cycles: an etch cycle and a passivation cycle. Thus, for each cycle a different gas is used. For instance, to etch silicon a gas such as sulfur hexafluoride $\left(\mathrm{SF}_{6}\right)$ is used for the etch cycle and a gas containing carbon and fluorine, such as carbon tetrafluoride $\left(\mathrm{CF}_{4}\right)$ or in our case octafluorocyclobutane $\left(\mathrm{C}_{4} \mathrm{~F}_{8}\right)$, is used for passivation.

In the passivation cycle a thin layer is deposited on the surface and sidewalls of the substrate. This layer acts as an inhibiter. In the etch cycle the inhibiter layer protects the sidewall from being etched while the layer on the bottom of the trench will be etched preferentially by the ion bombardment. The thickness of the inhibiter layer is very important in the final etch profile. If it is too thin, the sidewalls will be etched and will be tapered. If it is too thick, the passivation layer will prevent the substrate from being etched completely or at all. The alternating cycle forms scallops on the sidewalls, although the roughness of these scallops can be controlled to some extent by the operating conditions.

The Bosch process has become a common choice for deep etches with vertical sidewalls. Moreover, new sequences and chemistries have been explored including the addition of a third step in the cycle for depassivation of the trench bottoms or an isotropic etch cycle to improve the control on the sidewalls and profile as well as the process $[96,97,98]$.

Considering the etcher's design and the etch process, there are number of parameters that can be varied to obtain a desired etch profile. Chamber pressure, gas flow rate, RF power, duration of cycles, and temperature are the influential parameters in the parallel plate RIE etcher used in McMaster. 
Sh. Homampour - Masters Thesis

Engineering Physics, McMaster University

Chapter 3. Fabrication

\subsubsection{The Effect of the Parameters}

\section{Pressure}

Pressure in the chamber influences the concentration of free radicals in plasma. As the pressure is increased the mean free path of the atoms and molecules is decreased. Hence, there are more collisions and the probability of molecular dissociation is increased. As a result, the F concentration increases, which will increase the etch rate. However, further increasing of the pressure will reduce the ion energy and ion flux and as a result reduces the etch rate $[96,99,100]$.

To achieve a more anisotropic etch, one can decrease the pressure to increase the mean free path of the ions. This results in fewer ion collisions during the transit across the sheath ${ }^{3}$ and reduces sidewall etching due to the more highly directional arrival of ions at the surface. Alternatively, one can use the sidewall passivation technique to protect the sidewalls at high pressures.

Reduction of pressure will also increase the sheath voltage ${ }^{4}$ which results in more energetic ions and thus, a more directional etch. However, pressure reduction will decrease the chemical flux and the chemical etch rate. In addition, the plasma density will decrease, which will tend to lower the etch rate. The diffusivity of the species in the plasma is inversely proportional to the pressure. Hence, a reduction in pressure will increase the diffusivity which leads to improved uniformity of plasma. The more uniform plasma provides a better ion flux distribution and more uniform etch $[2,5,93,101]$. In RIE systems each parameter affects the others differently which makes the control of the system complicated.

\footnotetext{
${ }^{3}$ Sheath is the region next to each electrode where the voltage drop between plasma and electrode occur. Also corresponds to the dark regions of the plasma [2, 5]

${ }^{4}$ The voltage drop between plasma and electrodes [2]
} 
Sh. Homampour - Masters Thesis

Engineering Physics, McMaster University

Chapter 3. Fabrication

\section{Gas flow rate}

The flow rate of gases in different cycles affects the etch differently. Increasing the flow rate of the etch gas increases the etch rate since the concentration of the etching species, $\mathrm{F}$, increases and redeposition of the etch products $\left(\mathrm{SiF}_{4}\right)$ decreases. An increase of the flow rate in the passivation cycle decreases the etch rate due to a thicker passivation layer. Furthermore, as the flow rate increases, the anisotropy of the etch reduces due to enhancement of the chemical component of the etch. The pressure also increases with increased the flow rate $[86,96,102]$.

\section{RF power}

The RF power is one of the most influential parameters in the etch process. By changing the power by only a few watts an obvious change in the sidewall's slope is visible. RF power changes the ion energy directly and therefore, changes the etch rate. An increase in power increases the sheath voltage, which leads to an increase in ion energy, ion flux, and the fraction of the physical component of the etch. Moreover, the ion concentration in the plasma is proportional to the plasma density which increases with power.

\section{Cycle duration}

Similar to the flow rate of the gases, their cycle duration influences the etch profile as well. The longer the duration of the etch cycle is, the longer is the exposure of the sample to glow discharge and etching species and therefore, the greater the etch rate. However, too long an etch cycle will promote tapered sidewalls. On the other hand, the longer passivation cycle means thicker fluorocarbon film depositions on the sidewalls and substrate which decreases the etch rate and can also affect the slope of the sidewalls and surface roughness [96]. 
Sh. Homampour - Masters Thesis

Engineering Physics, McMaster University

Chapter 3. Fabrication

\section{Temperature}

Temperature of the substrate is normally kept at about 20-25 C. The lower the temperature is, the higher the deposition rate of the inhibitors on the sidewall is, and therefore the etch is more directional. It is important to minimize the temperature rise on the substrate's surface. In order to maintain a sufficient heat transfer between the substrate and the electrode backside helium pressurization is used $[2,96]$. 


\section{Chapter 4}

\section{Experimental Results}

\subsection{Dry Etch Characterization}

Dry etching of silicon is critical in the formation of silicon photonic circuits. Although an RIE system exists within the McMaster CEDT, it has not been qualified for use at the time of the commencement of this work. This section describes results which will be suitable for a wide range of etching applications, including silicon photonic device fabrication.

Silicon samples with dimensions $1 / 2 \times 1$ inch with photoresist and oxide masks were prepared to characterize the STS 320PC parallel plate etcher located at McMaster manufactured by Surface Technology Systems. The etcher has four recesses to hold samples, and the front recess was used in this investigation. Samples were placed in the holder after being bonded to a 4 inch silicon wafer ${ }^{1}$ with a solution of crystal bond and acetone. The feature thickness was measured with an alphastepper before the etch, right after the etch, and after removing the mask and clean-

\footnotetext{
${ }^{1}$ The wafer had a thick oxide layer on the surface to prevent etching of the wafer
} 
Sh. Homampour - Masters Thesis

Engineering Physics, McMaster University

Chapter 4. Experimental Results

ing the sample. $\mathrm{SF}_{6}$ gas was used as the etch gas, $\mathrm{C}_{4} \mathrm{~F}_{8}$ gas as the passivation gas, oxygen and hydrogen for purging between the two cycles steps. Previously, $\mathrm{CF}_{4}$ had been used in the passivation cycle but it was replaced with $\mathrm{C}_{4} \mathrm{~F}_{8}$ to increase the carbon content of the plasma and reduce the free $\mathrm{F}$ radicals to raise the selectivity between $\mathrm{Si}$ and $\mathrm{SiO}_{2}$ [2]. In addition, oxygen had been used, especially in continuous etch process, to reduce the lateral etch rate. By adding oxygen to an $\mathrm{SF}_{6}$ plasma, a chemically inert layer is produced that acts as an inhibitor and the anisotropic nature of the etch is improved [86].

This system was originally designed for continuous etch process in which all the gases are introduced to the chamber at the same time and the etch profile is determined by their relative flow rates and settings of the parameters. However, controlling the etch parameters in the continuous process is a difficult task. The etch tends to be isotropic and obtaining a vertical sidewall is difficult. Therefore, a Bosch process was designed for the system.

The $\mathrm{C}_{4} \mathrm{~F}_{8}$ flow rate was varied from 16 to $32 \mathrm{sccm}$, the power was varied from 50 to 200 Watts, the etch and passivation cycle times normally had the relative ratio of $2: 1$, but $1: 1$ and $4: 3$ ratios were also investigated. The $\mathrm{SF}_{6}$ flow rate was kept at $20 \mathrm{sccm}$, temperature at 25 degrees, and pressure at $20 \mathrm{mT}$. The flow rates were chosen based on the MFCs and ratios commonly used for such etchers in industry. Other parameters were chosen considering the suggested values by the company and common values. 
Sh. Homampour - Masters Thesis

Engineering Physics, McMaster University

Chapter 4. Experimental Results

\subsubsection{Analysis of Results}

\subsubsection{Effects of Power on Etch Rate and sidewall Angle}

The flow rate of $\mathrm{SF}_{6}$ was set at $20 \mathrm{sccm}$ and that of $\mathrm{C}_{4} \mathrm{~F}_{8}$ at $32 \mathrm{sccm}$ with cycle ratio of 2:1 respectively. The pressure was $20 \mathrm{mT}$ while temperature was kept at 25 degrees. The power was varied from $50 \mathrm{~W}$ to $200 \mathrm{~W}$ while etch rate was measured. The results are plotted in Figure 4.1. Note that the curves in the figures of this section are all to just guide the eye.

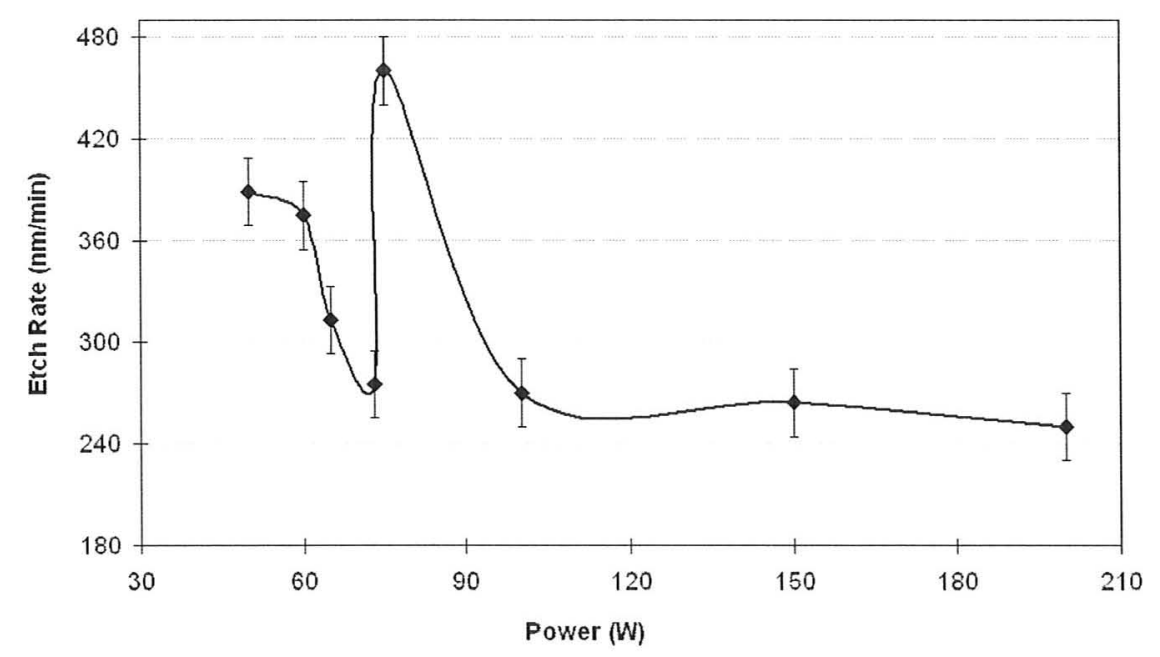

Figure 4.1: Graph of Etch Rate vs. Power

The slope of the sidewalls of each sample was also measured by taking its SEM picture and using ImageJ software. The obtained results are illustrated in Figure 4.2.

At low powers, i.e. from 50 to $75 \mathrm{~W}$, the chemical component of the etch is dominant, and therefore the etch rate is relatively low. The flux of the neutral chemical species with a wide angular distribution and collisions of the reactant species with the sides of the sample before arriving at the etch surface cause positively tapered 
Sh. Homampour - Masters Thesis

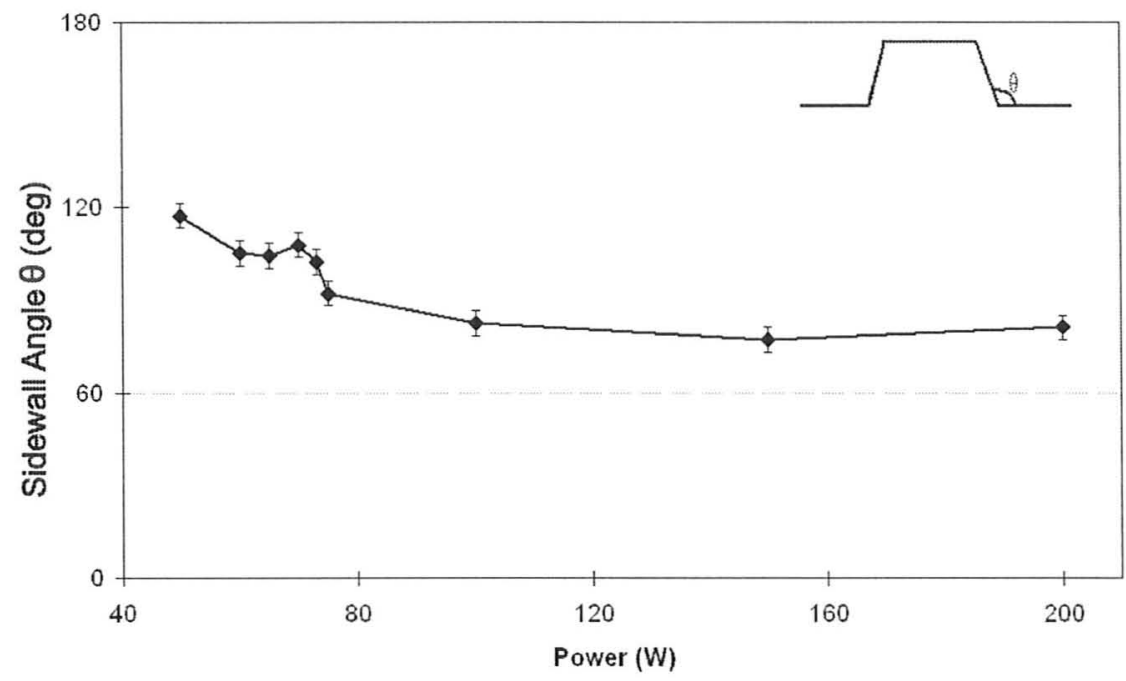

Figure 4.2: Graph of Sidewall Angle vs. Power

sidewalls.

As power reaches $75 \mathrm{~W}$ the best balance between the etch parameters is reached. The etch rate is at its highest value and sidewalls are vertical within \pm 2 degrees.

Raising the power above $75 \mathrm{~W}$ leads to reduction in etch rate and selectivity. Note that selectivity is obtained by comparison of the etch rates of different materials. Generally, increasing the RF power will enhance the ion flux and F radicals, which increases the etch rate until a peak is reached. Beyond this point, generation of $\mathrm{F}$ components does not depend on the power but is limited by the pressure. Thus, further increase of power does not increase the ion flux unless the pressure is increased to obtain a higher etch rate. Therefore, etch rate and anisotropy, i.e. having a vertical sidewalls, can be improved with raising power depending on the pressure in the chamber $[96,103]$.

Moreover, inspecting the sidewalls shows undercutting, which means ions have too much energy, the ion density and free radical concentration are too high which 
leads to lateral chemical etching of the walls, or free radicals sputter before they have time to react with the surface of the sample and are deposited on the sidewalls. The flux of the neutral species decreases with height, especially in narrow features with high aspect ratios. Hence, the sidewalls become undercut $[3,104,105]$. Similarly, the nonuniformity in the polymer deposited on the sidewalls will also result in undercut sidewalls. Thus, decreasing the RF power can reduce the ion energy and lowering the pressure can decrease the chemical flux and hence the chemical etch rate. Moreover, decreasing the pressure will increase ion energy, so decreasing power is helpful.

Reduction in the selectivity also confirms that the chemical component of the etch has changed since the selectivity depends on the chemical reactions and the proper proportions between the etch parameters. Finally, the combination of chemical and physical etching does not always act as predicted and there exist some irregularities.

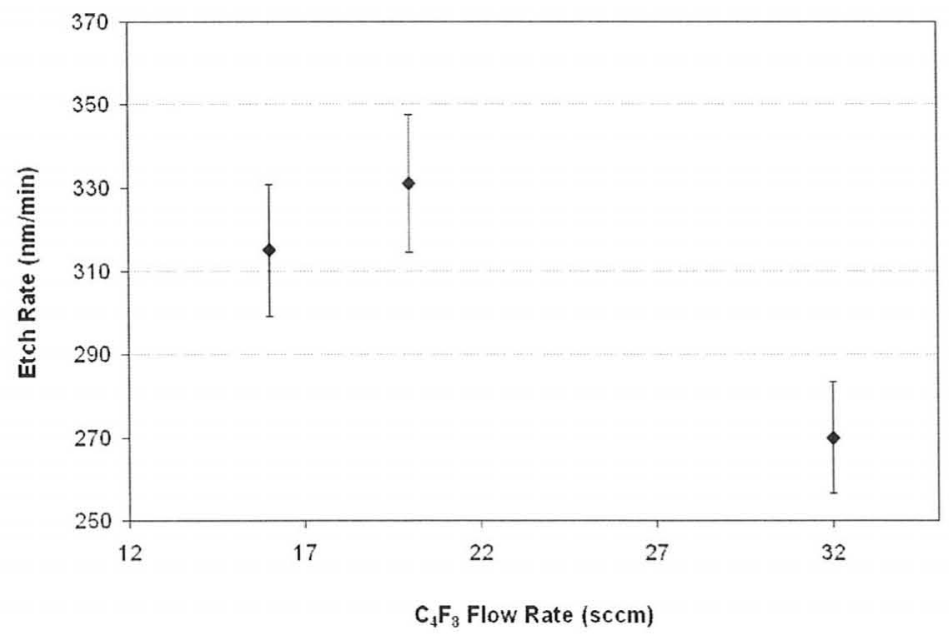

Figure 4.3: Graph of Etch Rate vs. $\mathrm{C}_{4} \mathrm{~F}_{8}$ Flow Rate. 
Sh. Homampour - Masters Thesis

\subsubsection{Effects of Passivation Cycle Flow Rate on Etch Rate and Sidewall}

\section{Angle}

The flow rate of $\mathrm{SF}_{6}$ was set at $20 \mathrm{sccm}$ with the etcher set to a passivation cycle ratio of 2:1. The pressure was $20 \mathrm{mT}$ while the temperature was kept at 25 degrees. The power was set at $100 \mathrm{~W}$ and the $\mathrm{C}_{4} \mathrm{~F}_{8}$ gas flow rate was varied from 16 to 32 sccm while the etch rate was measured. The results are plotted in Figure 4.3. The slope of the sidewalls of each sample was also measured with the same technique described in the previous section. The results are illustrated in Figure 4.4.

Generally, the higher the flow rate of the gas, the higher the etch rate but too high a flow rate can lead to a decrease in etch rate. Considering that in the Bosch process there are different cycles of gases, an etching gas and a passivation gas, the flow rate effect is consistent for the etch cycle gas but not for the passivation cycle.

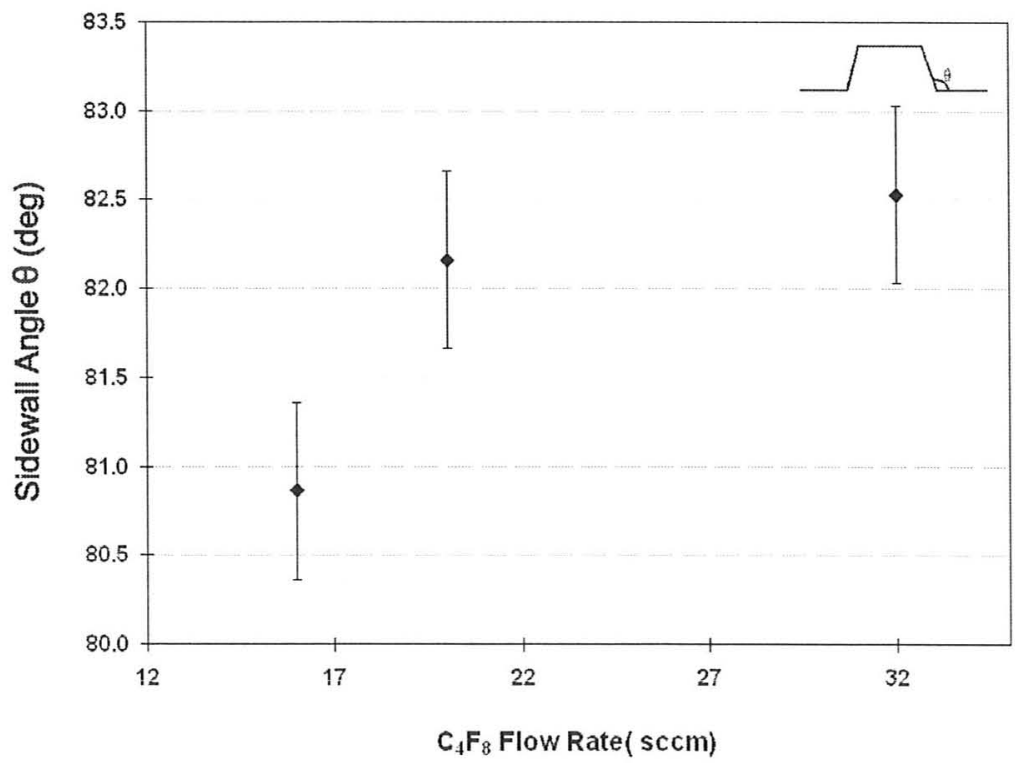

Figure 4.4: Graph of Sidewall Angle vs. $\mathrm{C}_{4} \mathrm{~F}_{8}$ Flow Rate.

The results show that the greater the flow rate of the passivation gas, the more 
Sh. Homampour - Masters Thesis

vertical the sidewalls are, but the etch rate decreases at maximum flow rate. The latter is due to the rise of the pressure as the result of the increase of the flow rate which yields to decrease in the etch rate. Sloped etch profiles are illustrated in Figures 4.5 and a vertical profile in Figure 4.6.
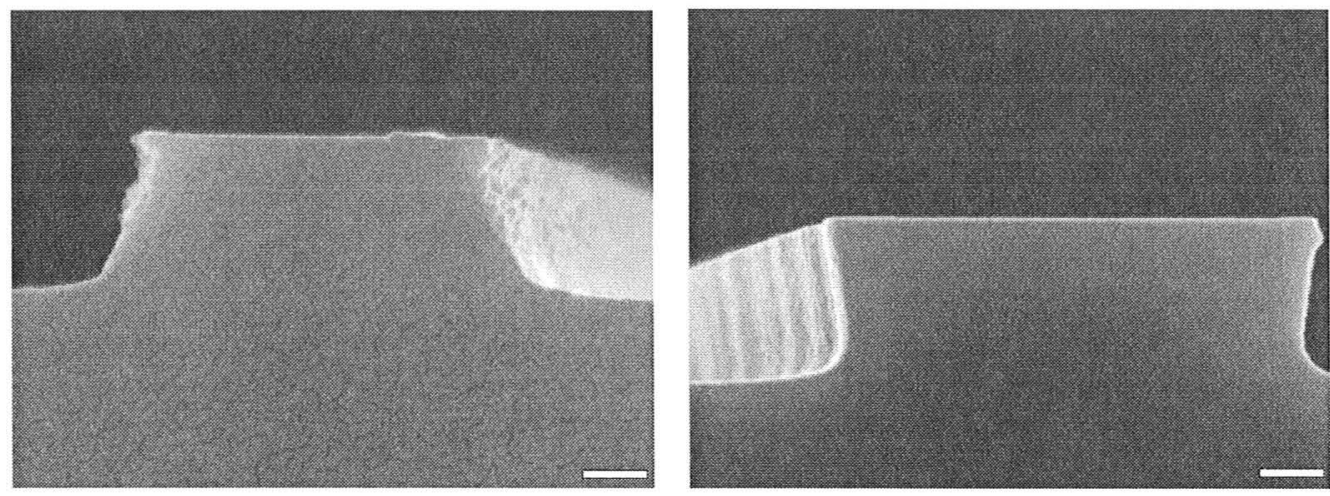

Figure 4.5: A positively tapered sidewall on left with $60 \mathrm{~W}$ power and A Sidewall with Undercut is illustrated on right with power set at $100 \mathrm{~W}$. The $\mathrm{C}_{4} \mathrm{~F}_{8}$ and $\mathrm{SF}_{6}$ flow rates were kept at 32 and $16 \mathrm{sccm}$ respectively. The white bar indicates $200 \mathrm{~nm}$.

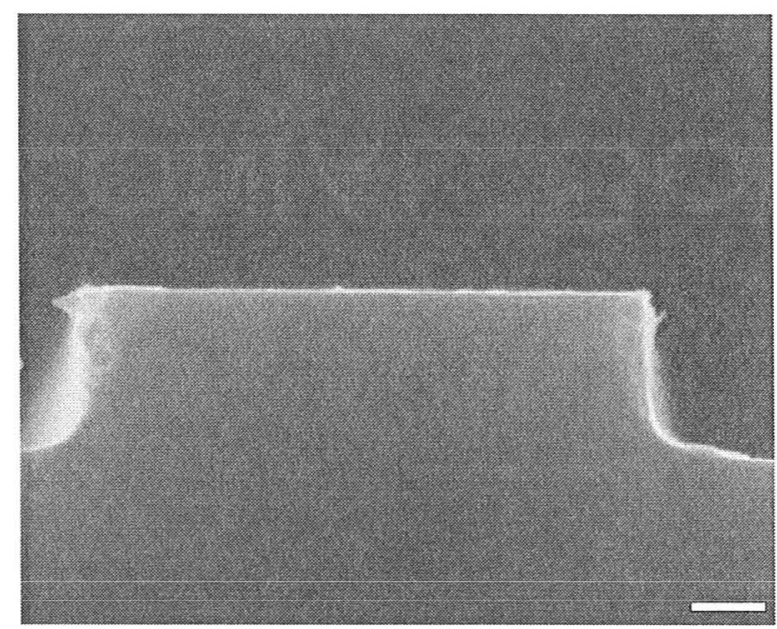

Figure 4.6: Vertical Sidewall with $75 \mathrm{~W}$ power. The flow rates were same as that in above figures. The white bar indicates $200 \mathrm{~nm}$. 
Sh. Homampour - Masters Thesis

Engineering Physics, McMaster University Chapter 4. Experimental Results

\subsubsection{Sidewall Roughness}

Sidewall roughness is one of the important aspects of an etched sample since it has direct influence on device performance. In MEMS, the roughness of the surface affects the other mechanical properties of the etched microstructure such as surface tension, while in optics sidewall smoothness is important in minimizing the loss due to light scattering. The surface roughness exists in both isotropic and anisotropic etches but arises for different reasons.

One of the obvious features of the Bosch process is the scalloping of the sidewalls due to the alternating between the cycles. Changing the etch parameters will enhance or diminish this feature.

Pressure is considered to be the dominant factor affecting surface roughness. Pressure influences the mean free path of the reacting species. An increase in pressure reduces the mean free path of the atoms and ions and the other species in the plasma and their path becomes less directional. Hence, the range of angles of incidence of the reactant species increases. Some of them arrive normal to the substrate while others are obliquely incident. The ions at oblique incident angles get reflected from the surface into the sidewalls at near normal angles which leads to more sidewall roughness. The same reasoning can be used to explain the undercut of the sidewalls at high pressures.

However, at the low pressures most of the ions arrive normal to the surface of the substrate, which prevents them from being reflected and thus, sidewalls are more smooth. It is important to note that the effect of the pressure is completely opposite to the roughness of the substrate's surface due to the opposite nature of the angle of the incidence of an ion on substrate's surface and sidewalls [103, 106].

RF power is one of the other parameters that impact the sidewall scalloping. A 
Sh. Homampour - Masters Thesis

Engineering Physics, McMaster University Chapter 4. Experimental Results

change in the RF power induces a change in the ion flux and directionality of the ions and therefore their angle of incidence on the substrate's surface. Based on the same reasoning, the etch rate of the passivating film on the surface of the substrate and sidewalls is different which leads to a minimum amount for RF power where the etch rate of the planar film is higher than that of the sidewalls. Basically, this is the point beyond which the physical component of the etch becomes the dominant component $[103,106]$.

There are other modifications that can improve the sidewall roughness. One is minimizing the cycle duration. However, this will reduce the selectivity, which can be overcome to some extent by increasing the relative time ratio between the cycles [107]. Oxidation of the sidewalls after etching and then removing the oxide layer will also lead to a smoother morphology. The different rate of oxide's growth for concave and convex surfaces leads to this improvement [108].

\subsubsection{Uniformity}

The uniformity across the sample is controlled by the plasma uniformity and the temperature uniformity across the sample. Normally, a higher plasma density is achieved closer to the RF power source or heat source, which leads to higher etch rates at the edges of the sample compared to the center. Pressure influences the diffusivity of the elements and therefore, the uniformity of the plasma. The lower the pressure is, the higher the diffusivity is and thus the better is the distribution of the ion flux on the sample. Hence, a low flow rate of the etch gas is desired to maintain a low pressure [96]. 
Sh. Homampour - Masters Thesis

Engineering Physics, McMaster University Chapter 4. Experimental Results

\subsubsection{Propagation Losses}

The propagation loss of the etched waveguides with vertical sidewalls was measured to be $-12.5(\mathrm{~dB} / \mathrm{cm})$ with standard deviation of 2.3 . The dominant cause of the losses is considered to be the surface roughness, followed by the uniformity over the entire waveguide. The measurements were conducted using the optical setup described in section 4.2.1 without the copper block and the temperature controller.

\subsection{Thermal Tuning of the Resonant Wavelength of the Ion Implanted Planar Bragg Gratings}

\subsubsection{External Heater}

An external thermoelectric heater was used in the measurements to control the temperature of a copper mounting block. The heater and a monitoring thermocouple were connected to a Newport temperature controller model 3150.

\subsubsection{Experimental Setup for Optical Characterization}

A sample with $\mathrm{Si}^{+}$ions implanted at $50 \mathrm{KeV}$ energy and 10 grating lengths ranging from 350 to $2100 \mu \mathrm{m}$ was prepared for measurements. The rib width was $2.2 \mu \mathrm{m}$ and the silicon ridge layer was $2.5 \mu \mathrm{m}$ thick at its center. The ribs were etched to a depth of $\sim 700 \mathrm{~nm}$. Five implant-free straight waveguides were also etched in the sample to provide a baseline for the measurement of the excess loss caused by the insertion of the implanted Bragg gratings.

The experimental setup illustrated in Figure 4.7 was used to obtain the transmission spectra of the Bragg gratings in a rib waveguide for TE and TM light. At 
first the measurement was done at room temperature and then repeated at higher temperatures.

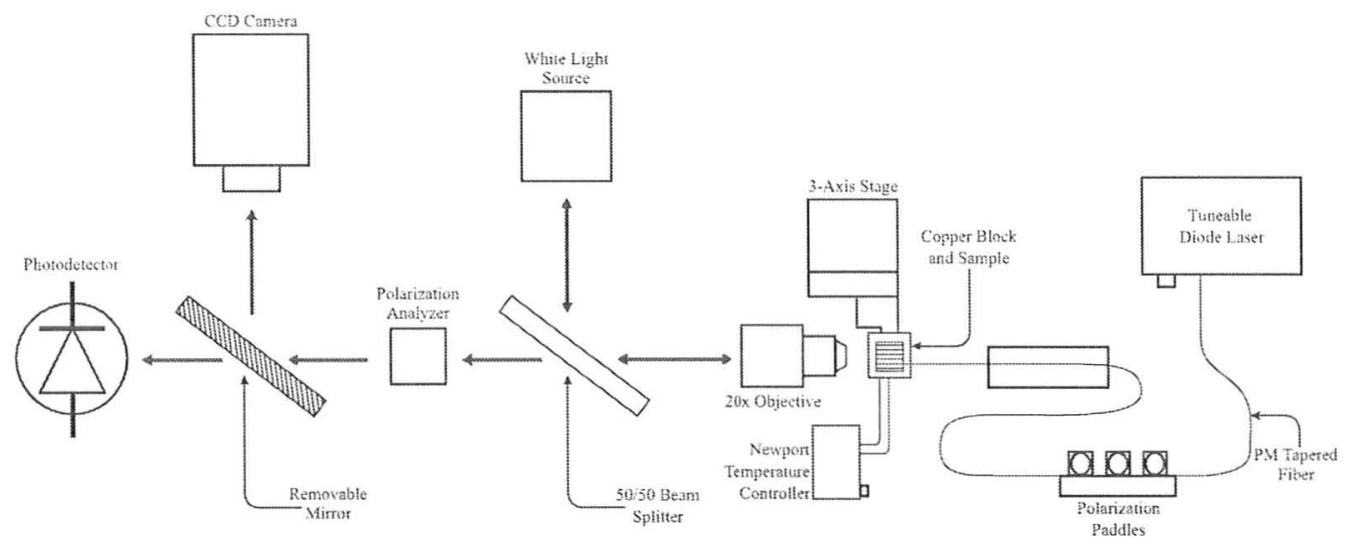

Figure 4.7: Experimental setup for optical characterization of samples with external heater.

The tunable laser was fiber-coupled to a three-paddle polarization controller. The tilt of each paddle of the polarizer could be altered to change the stress-induced birefringence acting on the looped fiber. Hence, the state of polarization, TE or TM, was set by the polarization analyzer. The polarized light was then fed through a polarization-maintaining (PM) single mode tapered fiber to slowly reduce the mode size to about $2.5 \mu \mathrm{m}$ while preserving the light's polarization. The reduction of the mode's size decreases the optical losses due to mode mismatch between that of the fiber and the rib waveguide and improves coupling dramatically.

The copper block connected to the temperature controller was placed on the stage and the sample was sited on the block. The fiber was butt coupled to the rib waveguide facet.

The output light was collected from the waveguide's facet via $20 \mathrm{X}$ objective lens and then directed towards either the CCD infrared camera to capture light for alignment purposes and image the mode or the InGaAs photodetector to measure 
Sh. Homampour - Masters Thesis

Engineering Physics, McMaster University Chapter 4. Experimental Results

the transmitted power. The $50 / 50$ beam splitter and the white light source were used only to align the setup.

\subsubsection{Transmission Spectra}

The transmission spectra of TE and TM modes of the sample ion implanted with $50 \mathrm{KeV}$ energy were first measured at room temperature $\sim 22^{\circ} \mathrm{C}$. The measurement was done by scanning the tunable diode laser from $1520-1570 \mathrm{~nm}$ with a wavelength step of $0.1 \mathrm{~nm}$. The measured transmission spectra were then normalized to the corresponding throughput power to establish a grating strength trend. A typical transmission spectrum is illustrated in Figure 4.8.

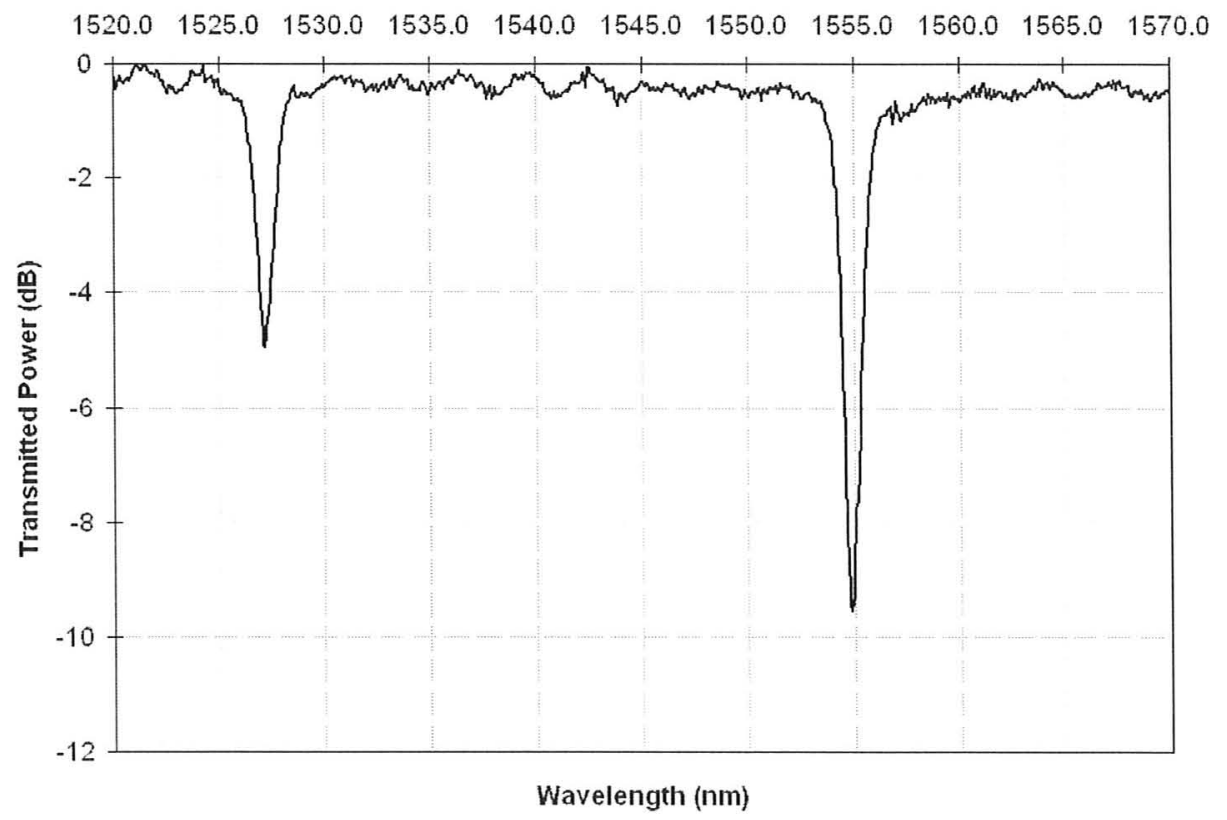

Figure 4.8: The typical transmission spectrum of rib waveguides with implanted Bragg gratings

Considering the governing equation for the Bragg period, only one Bragg wavelength is expected to be reflected in a single mode waveguide and therefore, only 
Sh. Homampour - Masters Thesis

Engineering Physics, McMaster University Chapter 4. Experimental Results

one dip at $\sim 1550 \mathrm{~nm}$ is anticipated in the transmission spectrum. However, two distinct dips were observed in the spectral scans for the SOI waveguides, as shown in Figure 4.8 .

The longer wavelength dip at $\sim 1555 \mathrm{~nm}$ corresponds to the coupling between the forward and backward traveling fundamental modes as predicted, while the shorter wavelength dip is due to the coupling between the forward traveling fundamental mode and the first backward traveling higher-order leaky mode [109]. Since the rib waveguide is a single mode structure, higher order modes are not supported and will radiate out of the rib over a short propagation distance. The wavelengths corresponding to the coupling modes can be computed using the phase matching condition and the eigenvalue equation for total phase shift of the asymmetrical planar waveguides [5] as discussed in section 2.2.2.

The fundamental Bragg wavelength was measured to be $\sim 1554.6 \mathrm{~nm}$ instead of the anticipated resonant wavelength at $1550 \mathrm{~nm}$. This slight deviation can be attributed to a minor angular misalignment of the Lloyd's mirror setup since $1^{\circ}$ angular deviation leads to $\mathrm{a} \sim 25 \mathrm{~nm}$ change in the resonant wavelength.

The transmission spectrum for the $2100 \mu \mathrm{m}$ long implanted Bragg grating exhibited a transmission reduction of $12.1 \mathrm{~dB}$ at the Bragg wavelength. However, the transmitted power for the rib waveguide with the same length Bragg gratings but higher implant energy showed greater extinction ratio due to the greater depth of the damage profile, thus, increased overlap between the grating and the mode. An extinction ratio of $18.1 \mathrm{~dB}$ [62] was achieved from the rib waveguide with the same grating length implanted but $60 \mathrm{KeV} \mathrm{Si}^{+}$ion energy.

Following the measurements at room temperature, the sample's temperature was raised via temperature controller in 10 degree intervals and after a 10 minute 
wait measurements were conducted. Only the TE mode was considered since the strength of the gratings and thus, Bragg reflection is greater than that of the TM mode as discussed in section 2.4. A positive shift in the resonant wavelength was induced and observed as shown in the Figure 4.9.

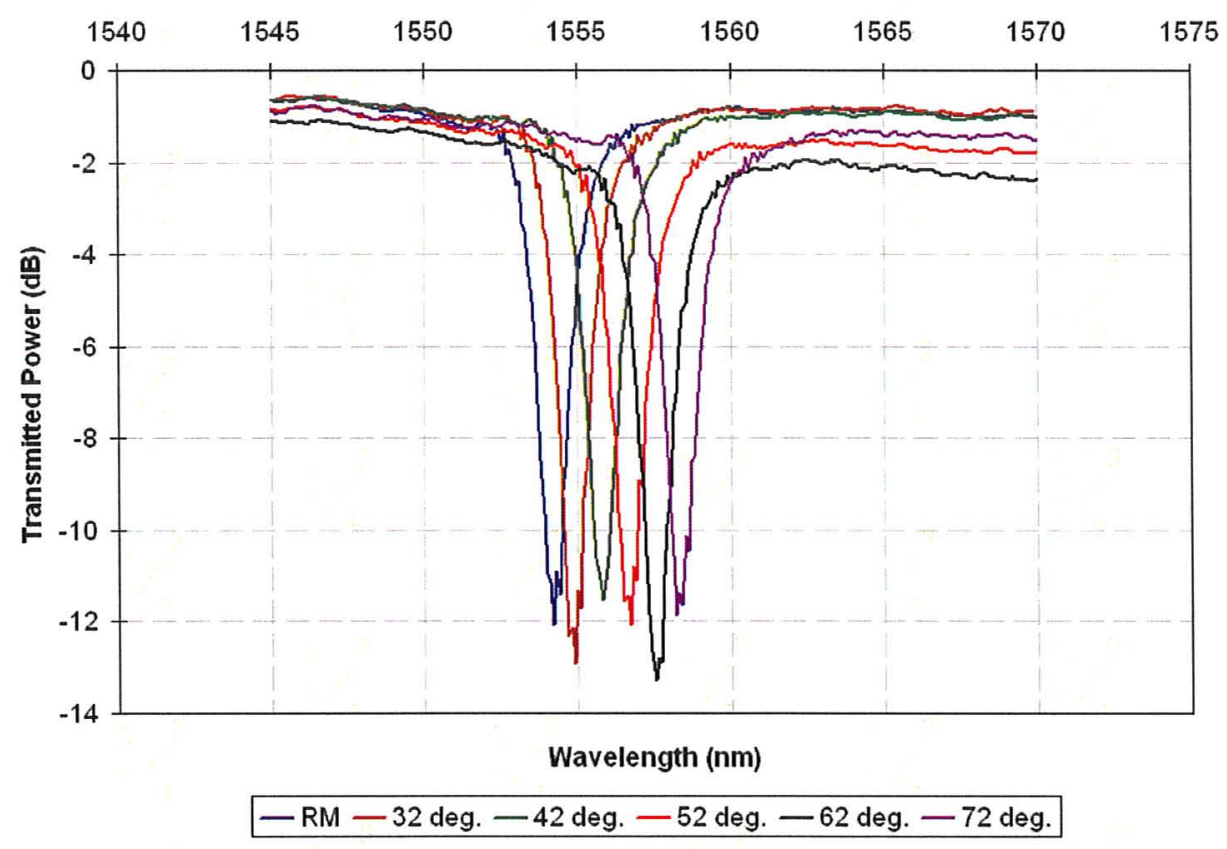

Figure 4.9: Transmission spectrum of planar Bragg grating rib waveguide for different temperatures.

The observed shift is of average $0.080 \mathrm{~nm} / \mathrm{deg}$ which agrees well with the theoretical prediction in section 2.5. The comparison is shown in Figure 4.10.

\subsubsection{Loss Measurements}

Many loss mechanisms are involved in optical propagation in rib wavguides. The most detrimental and prominent losses are the coupling losses due to mode mismatch and Fresnel reflections at input and output facets, and light scattering due to sidewall roughness of the structure. Moreover, fabrication procedures and ad- 


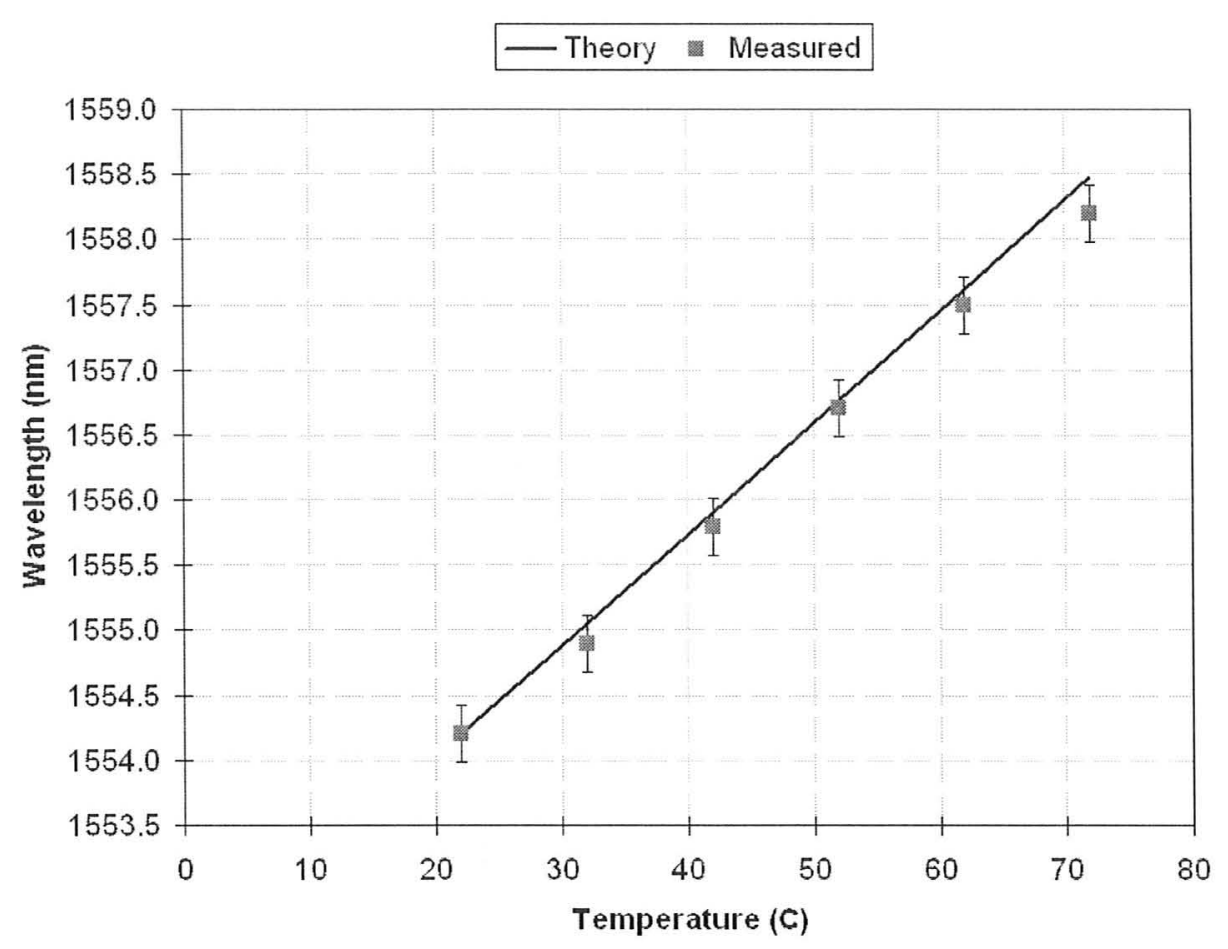

Figure 4.10: Measured and modeled shift in the resonant wavelength of the grating using $d \lambda / d T=((1 / n)(d n / d T+\alpha) \lambda$.

ditional layers and devices insert additional losses. Thus, by including a series of straight waveguides with the same dimensions in the device and implementing them as reference, such excess losses can be measured and accounted for. The excess loss due to implantation for the $50 \mathrm{KeV}$ implanted sample was determined to be $0.9 \mathrm{~dB} / \mathrm{mm}$ for TE polarization. The excess loss due to external heater and heating up the sample is not considered to be significant since the thermal expansion coefficient of silicon is on order of $10^{-6} \mathrm{~K}^{-1}$ [110]. Thus, 10 degrees temperature change results in length change of order $10^{-11} \mathrm{~m}$ in the device layer, which is negligible. 
Sh. Homampour - Masters Thesis

Engineering Physics, McMaster University Chapter 4. Experimental Results

\subsubsection{Integrated Heater}

\subsubsection{Experimental Setup for Optical Characterization}

Samples ion implanted with $\mathrm{Si}$ ions at $50 \mathrm{KeV}$ and $60 \mathrm{Kev}$ with 10 grating lengths ranging from 350 to $2100 \mu \mathrm{m}$, and aluminum strips and contact pads with 10 different lengths ranging from 550 to $2300 \mu \mathrm{m}$ were prepared for measurement. The rib widths were $2 \mu \mathrm{m}$ and the silicon ridge layer was $2.5 \mu \mathrm{m}$ thick at its center. The ribs were etched to a depth of $\sim 700 \mathrm{~nm}$. Five implant-free straight waveguides were also etched in the sample to measure the excess loss caused by the insertion of the implanted Bragg gratings and a complete set of ion implanted ribs including all the grating lengths without heaters was etched to measure the excess loss caused by the insertion of the aluminum heaters.

The experimental setup used was the same as that used for the external heater shown in Figure 4.7 with a minor difference i.e. the copper block was removed.

\subsubsection{Transmission Spectra}

Samples ion implanted with both 50 and $60 \mathrm{KeV}$ energies were measured. Most of the samples transmitted a relatively high amount of power (in few cases up to 20 $\mu W)$ but only a small Bragg grating dip was observed in the transmission power spectrum. The relevant spectrum is illustrated in Figure 4.11.

A typical spectrum obtained from other measurements is shown in the Figure 4.12. In these cases the observed mode in the CCD camera used to monitor the rib output profile, looked multimode (i.e. having two or more bright spots instead of one) which was not expected since the dimensions of rib waveguide were designed to assure a single mode operation. 
Sh. Homampour - Masters Thesis

Engineering Physics, McMaster University

Chapter 4. Experimental Results

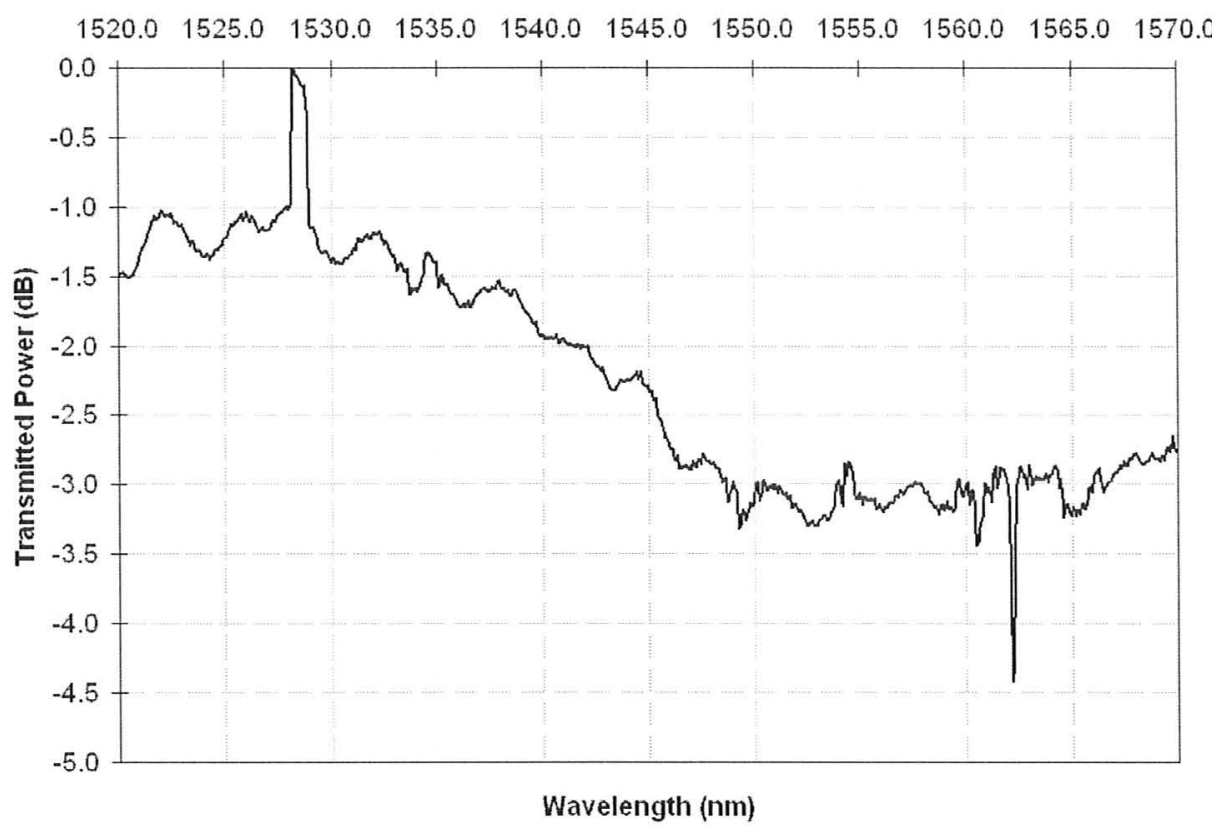

Figure 4.11: The transmission spectrum of an ion implanted sample with integrated aluminum heaters. A small dip close to the expected wavelength is observed.

The surface image of these samples were taken by SEM, one of which is shown in Figure 4.13.

As can be seen in the picture, the oxide layer in between the waveguide and aluminum layer did not remain in the same state at all times. Although the pattern of the aluminum is distinguishable, it seems that the aluminum and oxide layers are intermixed.

One possible cause of these surface imperfections is that the spin-on-glass layer did not reach a stable state due to the inability to heat the sample at temperatures higher than $150^{\circ} \mathrm{C}$. However, the SOG layer seemed intact when it was tested on trial samples; with electrical current passing through the aluminum layer with the application of voltage. Thus, the final step, i.e. removing the residual PR and using $\mathrm{O}_{2}$ plasma ash, could have influenced the SOG. 
Sh. Homampour - Masters Thesis

Engineering Physics, McMaster University

Chapter 4. Experimental Results

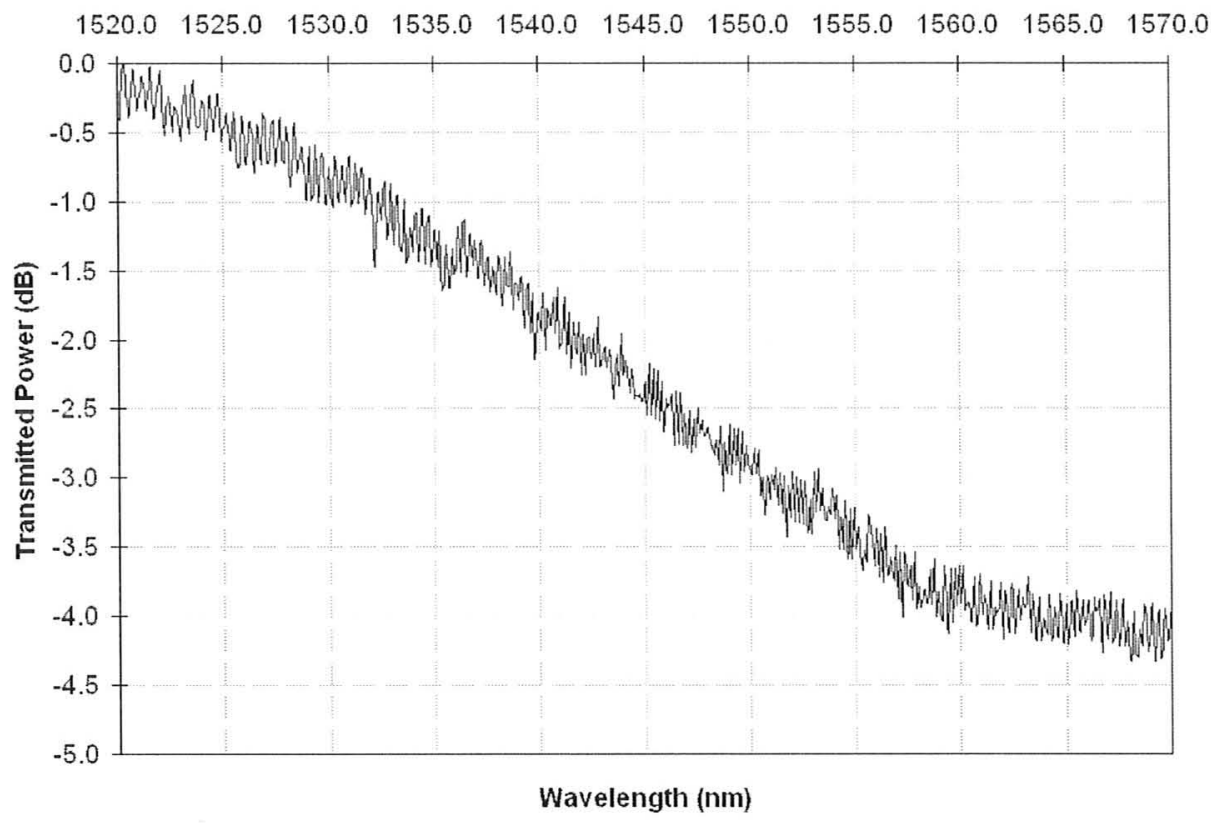

Figure 4.12: A transmission spectrum obtained from the ion implanted samples with integrated heater showing no bragg dip.

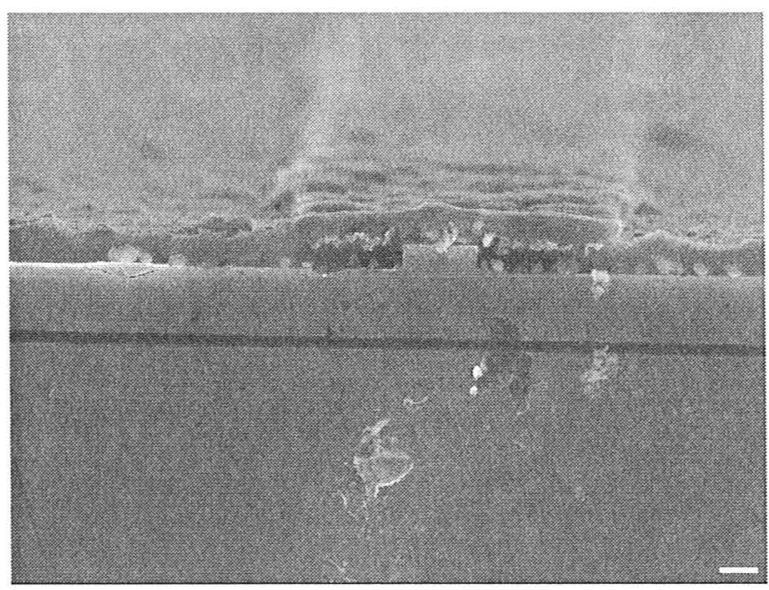

Figure 4.13: SEM image of an ion implanted rib with aluminum pattern and SOG. The white bar indicates $1 \mu \mathrm{m}$ length. 


\subsubsection{Comparison to the Etched Gratings}

The etched grating samples also consisted of 10 grating lengths ranging from 350 to $2100 \mu \mathrm{m}$ and aluminum strips and contact pads with 10 different lengths ranging from 550 to $2300 \mu \mathrm{m}$. The spectra of these samples looked better than that of the ion implanted samples. The modes looked single mode on the CCD camera and in many of the transmission spectra, two dips could be almost identified. An example of such a spectrum is illustrated in Figure 4.14.

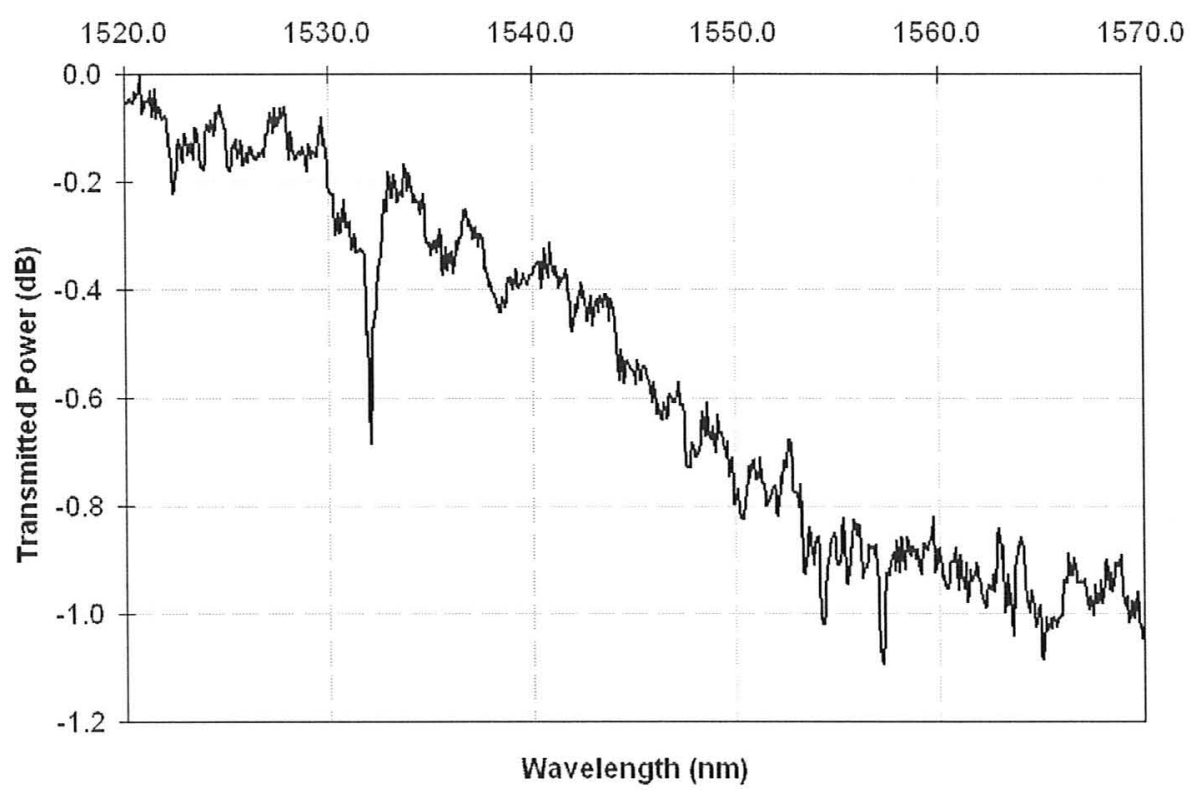

Figure 4.14: A TE mode transmission power spectrum of an etched grating.

However, application of voltage to the aluminum strips did not result in circulation of any current through the layer. The SEM picture of one of these samples, shown in Figure 4.15, looks very similar to that of the ion implanted ones.

Due to the complications involved in adding an oxide layer after the ion implantation steps, other device structures were considered. Addition of aluminum heaters 


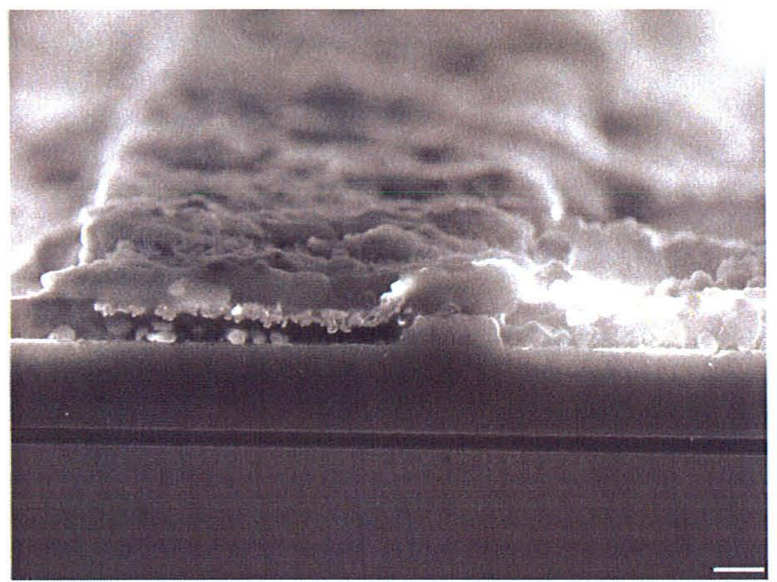

Figure 4.15: SEM image of an etched grating sample with aluminum pattern and SOG. The white bar indicates $1 \mu \mathrm{m}$ length.

on the side of rib waveguides is one of the possibilities. In this case no oxide layer is required, and based on the simulations done with BPM a minimum of $0.5 \mu \mathrm{m}$ between the metal on the rib is adequate to prevent optical losses due to the metal. A contour map of the rib waveguide mode is shown in Figure 4.16.

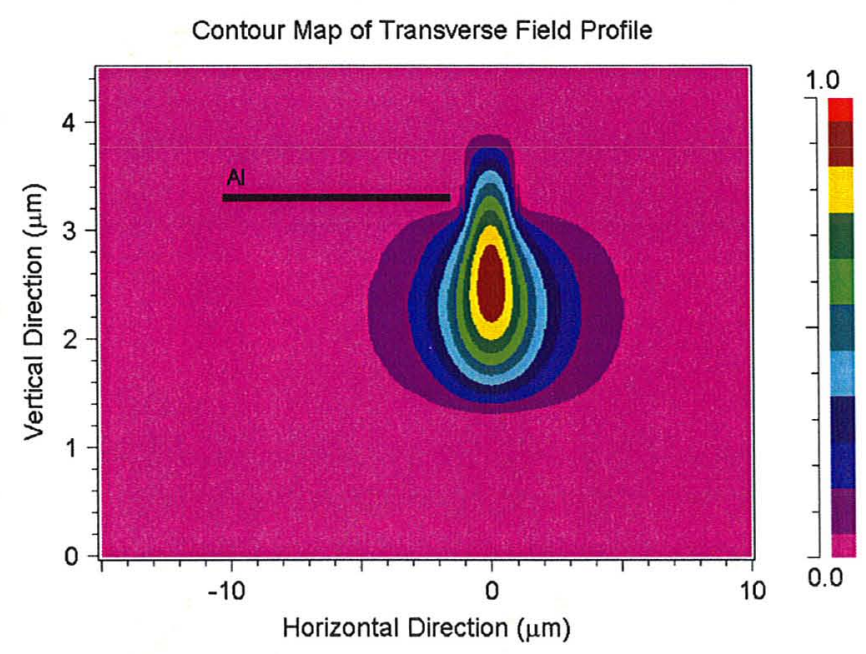

Figure 4.16: Contour map of the field when aluminum strip is deposited $0.5 \mu \mathrm{m}$ from a rib waveguide 
Sh. Homampour - Masters Thesis

Engineering Physics, McMaster University

Chapter 4. Experimental Results

It shows that the mode power at the surface of the silicon is very small in the etched slab regions, even as close to the rib as $0.5 \mu \mathrm{m}$. Thus a metallic heater could be placed beside the rib without using an oxide layer. The heat transfer properties of this structure were simulated by COMSOL, showing that application of $200 \mathrm{~mA}$ current to aluminum strips of $10 \mu \mathrm{m}$ wide, with a thickness of $300 \mathrm{~nm}$ leads to 15 degrees temperature increase relative to an ambient of $295 \mathrm{~K}$. The heat transfer profile and the temperature variation across the rib is illustrated in Figures 4.17 and 4.18.

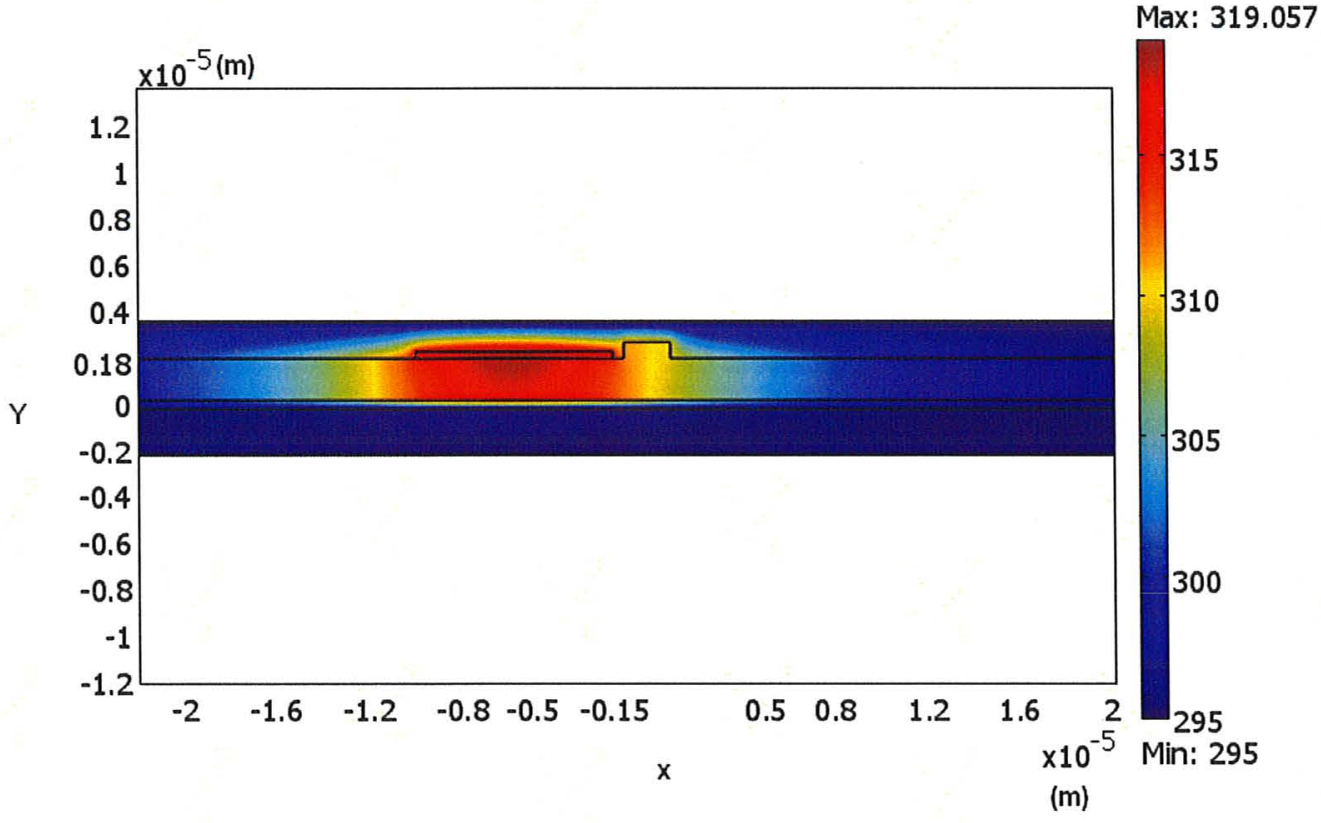

Figure 4.17: The heat transfer profile 

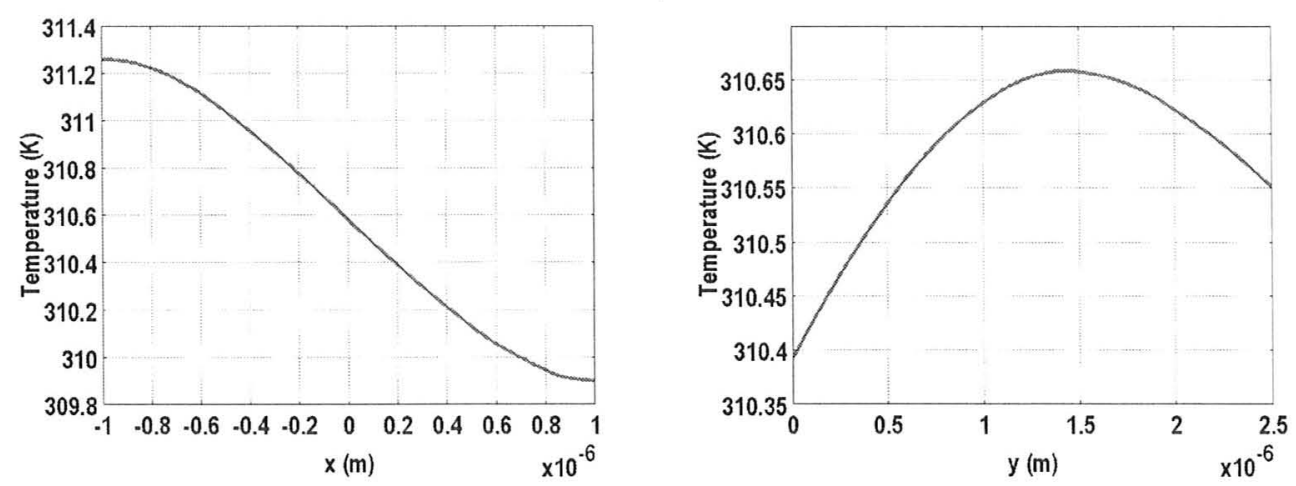

Figure 4.18: The plot on left shows the temperature variance across the width of the rib at $y=2.3 \mu \mathrm{m}$ and the variation of temperature from bottom to top of the rib at $x=0$ is shown on the one on right.

We compared the mode profile and heat transfer properties for the case of heaters beside the rib, with no oxide layer, to the case of heaters plus oxide on top of the rib. The latter was found to have more uniform lateral temperature distribution within the rib ( 0.25 degrees difference between the opposite edges of the rib). However, the former produced a more uniform temperature distribution vertically.

Another possible structure is to add aluminum heaters on both sides of a rib waveguide. Although, this enhances the heating procedure and results in more increase in temperature, it imposes more restrictions on the distances. The heat profile and plots of the temperature variation is shown in Figure 4.19 and 4.20. 
Sh. Homampour - Masters Thesis

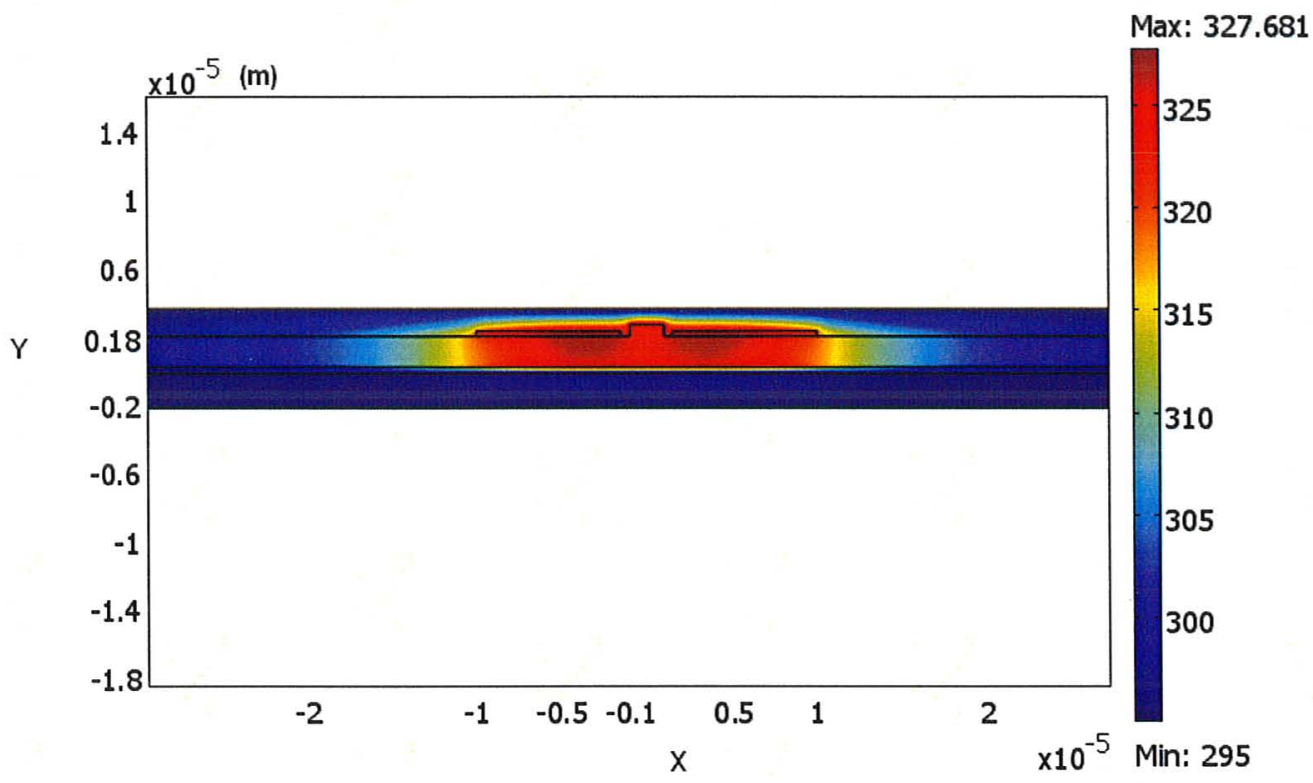

(m)

Figure 4.19: The heat transfer profile.
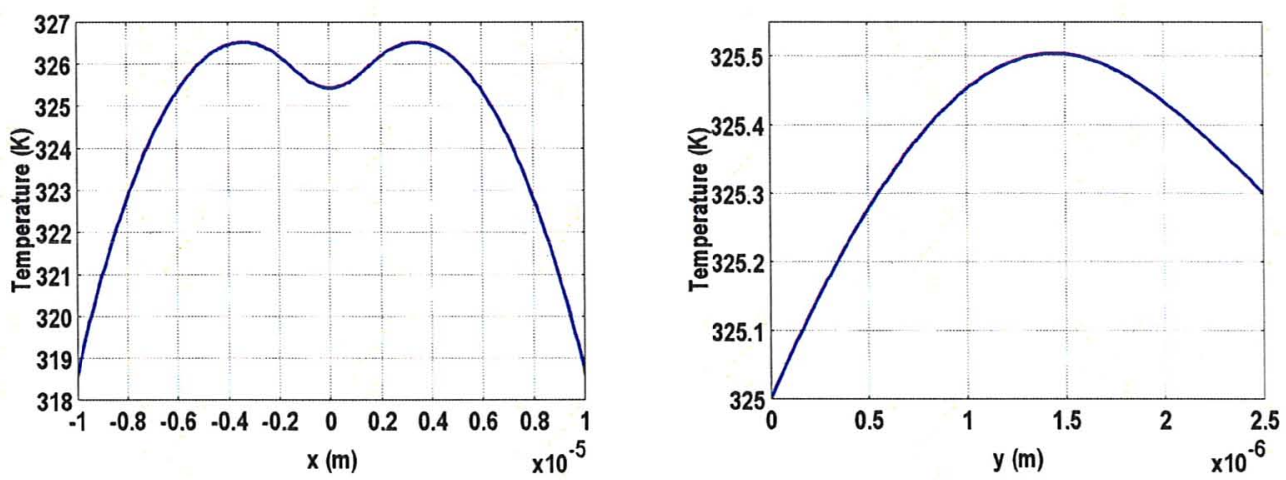

Figure 4.20: The plot on left shows the temperature variance across the width of the rib and the variation of temperature from bottom to top of the rib is shown on the one on right.

It is observed that the heat rise and heat uniformity is greater in this case as expected,while mode profile is less uniform. The contour of the rib waveguide mode of this structure is presented in Figure 4.21. Therefore, the two suggested 
structures have the potential to substitute the fabricated device while considering the constraints.

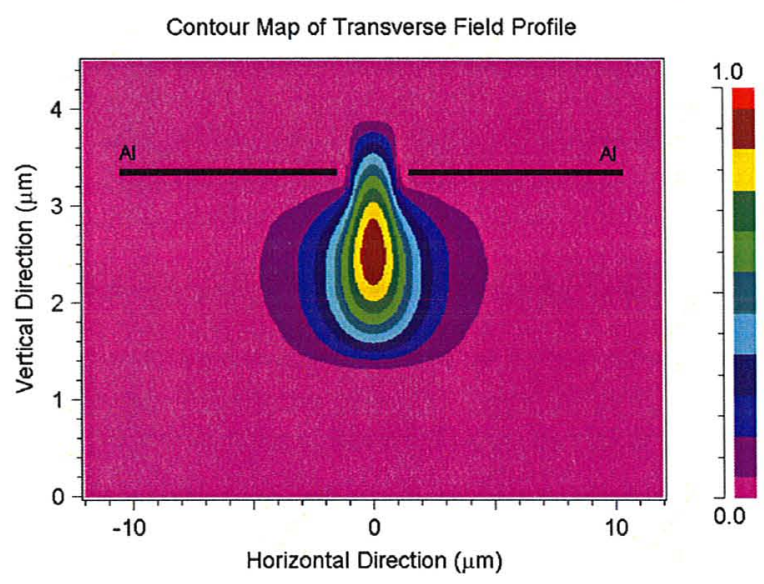

Figure 4.21: The contour map of the field when aluminum strips are deposited on each side of the rib at a $0.5 \mu \mathrm{m}$ from the rib waveguide.

\subsection{Broadband Grating Coupler on SOI}

A sample consisting of regions of etched gratings on slab SOI waveguides and adjacent unetched shiny regions was prepared as discussed in section 3.6. The sample was characterized using a setup similar to that shown in Figure 4.7 with small modification. The copper block and temperature controller were removed and a cleaved fiber connected to a xyz-stage was mounted vertically above the gratings, with motion in all three directions. This setup is illustrated in Figure 4.22

The tunable diode laser and horizontal fiber were used to align the setup and ensure that slab guided light was coupled to the detector. Once the detector alignment was confirmed, the power to the horizontal fiber was removed and the vertical fiber was brought very close to the surface of the sample to direct light onto the surface. The vertical fiber was connected to a diode laser operating at wavelength of 1550 

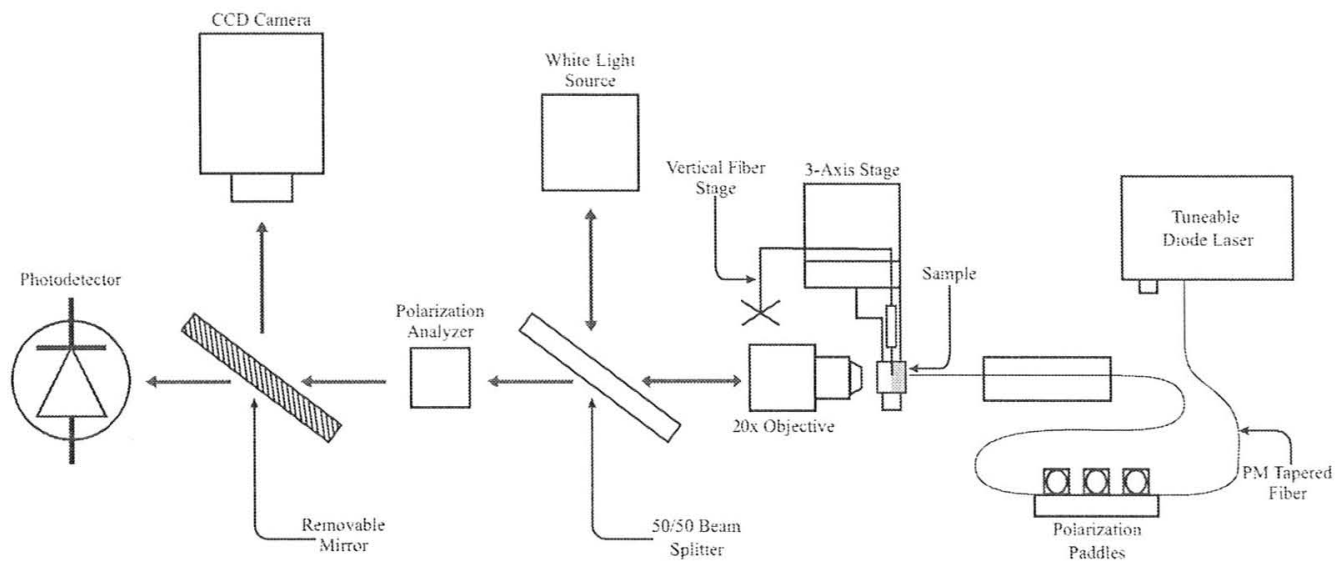

Figure 4.22: Experimental setup for optical characterization of the broadband grating coupler.

nm. The fiber was moved from the grating region to the unetched shiny side on a straight line and the transmitted power was measured at each point. The measurement was conducted on different parts of the sample. A typical plot obtained in the measurements is shown in Figure 4.23.

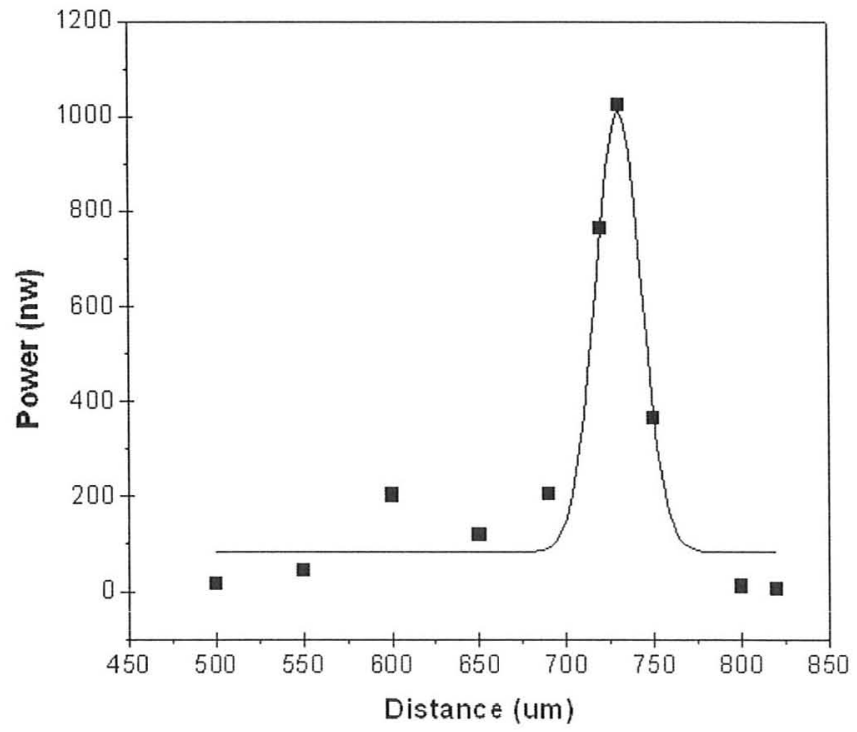

Figure 4.23: A typical plot obtained in the measurements of the broadband grating coupler with a fitted gaussian 
Sh. Homampour - Masters Thesis

Engineering Physics, McMaster University

Chapter 4. Experimental Results

The peak of transmitted power occurs when the fiber is at the border between the grating and the unetched region. As the vertical fiber is moved onto the unetched Si (i.e. more to the left on the graph) no coupling is expected thus, the power drops quickly to almost zero. On the other hand, as the fiber is moved deeper in the grating part, the intensity drops due to the out-coupling of the light that is incoupled below the fiber by the unilluminated parts of the grating. To compare the measurements, the logarithm of the detected power was plotted vs. the distance and then fitted with a straight line. The slope of the plot indicates the $1 / e$ decay length. Two examples of the measurements and the summary of all the measurements with the comparison between the measured and the estimated results is shown in Figure 4.24. Considering the average slope of the measurements and its standard deviation, the measurements and theory are in a good agreement. 
Sh. Homampour - Masters Thesis
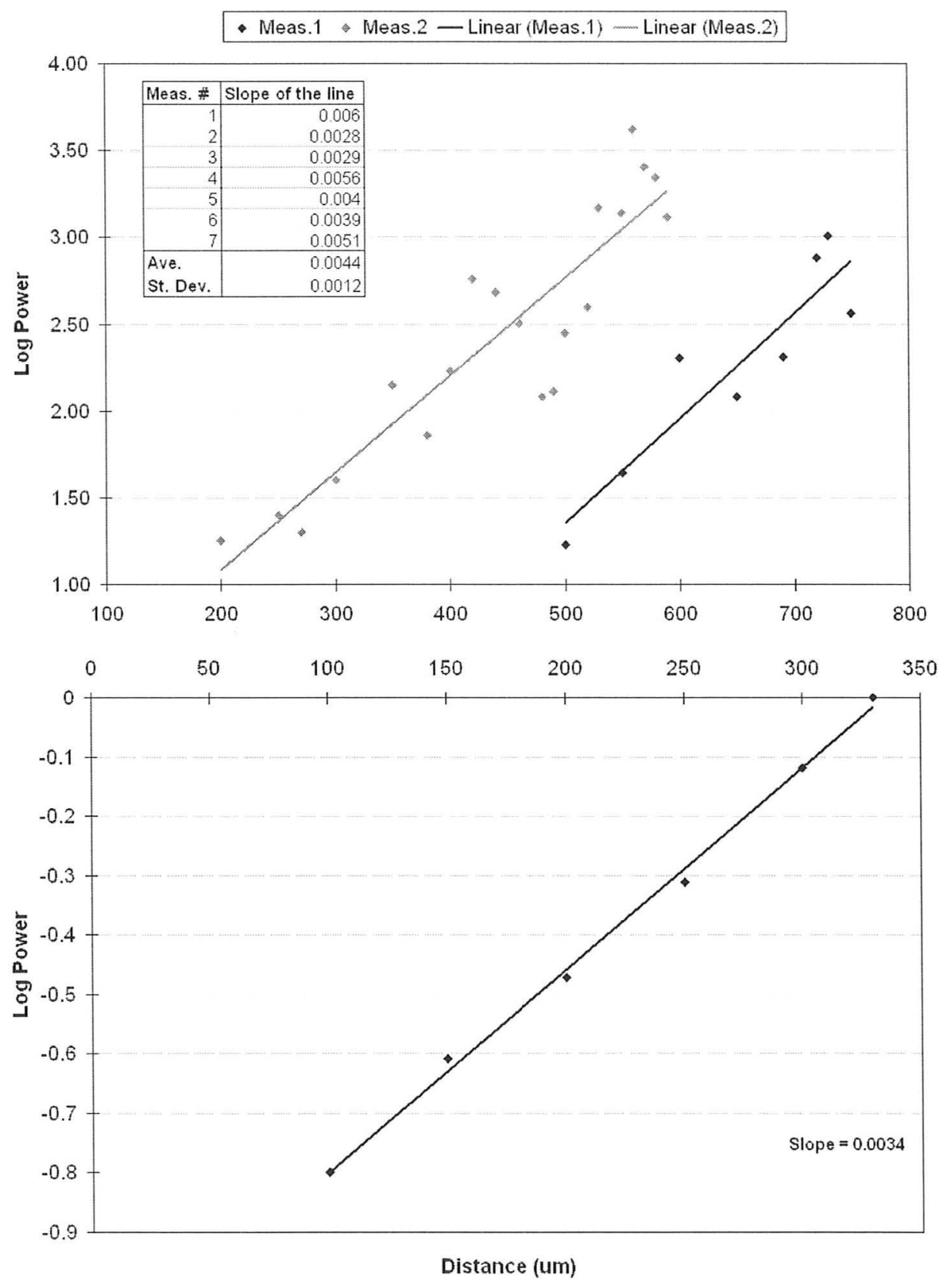

Figure 4.24: The summary of the measurements is shown in top and the theory in the bottom plot. The distance is the amount traveled from the border between the grating and unetched region. 


\section{Chapter 5}

\section{Conclusion}

Thermal tuning of implanted Bragg gratings in SOI rib waveguides is presented in this work. To our knowledge this is the first time such work is reported for silicon rib waveguides. Both external and integrated heaters were examined. By employing an external heater to increase the temperature, a positive shift of $0.080 \mathrm{~nm} / \mathrm{deg}$ in the resonant wavelength of planar Bragg gratings was observed. The observed shift was in good agreement with the positive shift of $0.082 \mathrm{~nm} / \mathrm{deg}$ predicted by theory, showing the tunability of planar Bragg gratings. Furthermore, the observed shift agrees with the recent report on thermal tuning of relief gratings [78].

The integrated thermal tuning and utilization of integrated heaters was also attempted using aluminum resistive heaters. However, the predicted results were not achieved due to the unstable state of the oxide layer resulting in a mixture of oxide and aluminum layers. The resulting complications are considered to be due to the temperature sensitivity of the implanted ions and the high temperatures involved in growing oxide layers and curing spin-on-glass.

Thermal tuning and more importantly integrated thermal tuning of the reso- 
Sh. Homampour - Masters Thesis

nance wavelength of implanted Bragg gratings in SOI rib waveguides introduces a new level of optical integration and efficiency in a silicon photonic platform. Thus, exploring other possibilities to replace the spin on glass layer, other methods of introducing oxide layer or replacing the oxide layer with another insulating layer would be very beneficial. Altering the oxide layer can also be beneficial for preparation of the facets since the thinning and cleaving method leads to better results in comparison to using a dicing saw. The two suggested structures can also substitute the existing structure and overcome the complexity of the oxide layer since it does not require an oxide layer. Hence, by overcoming the fabrication difficulties thermal tuning of planar Bragg gratings is very possible.

The initial measurements conducted on a Bragg grating coupler on a multimode planar SOI waveguide gave results in good agreement with the theory, showing its high potential to be used in integrated optical devices, predominantly in solar cells. The Bragg grating coupler can be a good alternative for coupling normal incident light into a substrate with less dependence on its ambient temperature and higher efficiency. More detailed studies on the coupling angle of light and the required period and shape of the gratings can be very valuable in reducing losses and improving the efficiency.

The parallel plate etcher at McMaster university was characterized to be used for the Bosch process, although this kind of etcher is not normally used for such processes. It was shown that by changing the combination of gases, adding steps to the process, and changing the influential parameters such as RF power, pressure, and gas flow the etcher is operable for advanced processes (such as Bosch) and that despite a longer time required for completion of each step, in comparison to more modern etchers, any sidewall angle may be achievable. However, further optimiza- 
Sh. Homampour - Masters Thesis

Engineering Physics, McMaster University

Chapter 5. Conclusion

tion and testing of new processes for expanding the possible profiles realizable with the etcher would be beneficial. 


\section{Appendix A}

\section{Conferences and Publications}

The following publications/ presentations have resulted from this thesis research.

- Sh. Homampour, M. P. Bulk, P. E. Jessop, and A. P. Knights, Thermal tuning of planar Bragg gratings in silicon-on-insulator rib waveguides", Physics Status Solidi (C), 6, S240-S243, 2009.

- Photonics North and CIPI conference, Quebec City, QC - May, 2009.

- ICOOPMA, Edmonton, AB - July, 2008.

(Received the Best Poster Award) 


\section{Appendix A}

\section{How to Operate the Etcher STS 320PC}

The STS 320PC parallel etcher is located at the first floor of the Tandem Accelerator Building (TAB). The operating procedure can be divided into three steps.

\section{Step 1. Powering Up the Etcher}

To get the etcher ready to run, the steps must be followed respectively.

1. Flip the breaker located on the wall behind the etcher to ON position.

2. Plug in the black cable of the power source.

3. Move to the gas controllers located on the wall to the far right side of the breaker and turn on the $\mathrm{N}_{2}$ and $\mathrm{O}_{2}$ gas knobs by turning them counter clockwise.

4. Move to the blue locker beside the etcher and open the $\mathrm{N}_{2}, \mathrm{SF}_{6}$, and $\mathrm{C}_{4} \mathrm{~F}_{8}$ gas cylinders. Ensure to open the black knobs on the tanks as well.

5. Open the $\mathrm{O}_{2}$ gas cylinder located beside the blue locker in the same manner as point 4.

6. Turn on the BetterTech Chiller i.e. the small box to the left of the etcher.

7. Turn on the etcher by pressing the power button.

\section{Step 2. Preparing the Computer for an Etch}


Sh. Homampour - Masters Thesis

1. Turn on the PC and select the first boot up option.

2. On the command prompt type : cd fdos/320 pc

3. Type process. Note that at this point the etcher's pumps must turn on. If they do not, most probably the power source of the etcher is experiencing a problem like a burnt part and should not proceed to the next step.

4. At this point a blue screen with the available recipes must be on the blue screen. Choose the required recipe.

5. After system is prepared, it indicates ready and F1, F2, F3, F4 commands indicated at the bottom of the page will be available.

\section{Step 3. Loading the Sample}

1. Press F3 (Vent) and subsequently F1 (Open).

2. Move to the etcher, press and hold the two matching buttons glowing white light located on right and left in front of the machine. Hold the button till the lid is completely open.

3. Load the sample in one of the four wafer holders, indicated as slightly shaded regions with an edge. The front one has shown more uniform results and is recommended.

4. Press F1 (close), press and hold the same buttons in step 2 till the lid is completely closed.

\section{Step 4. Etching}

1. Choose the recipe again on the screen.

2. When the machine is ready and the black screen is loaded, the instructions will be indicated on the screen. Press F1 to start the etch.

\section{Step 5. Preparing the Computer for Turning off}

1. After the etch process is completely finished, press F4 to exit the program. 
Sh. Homampour - Masters Thesis

2. When DOS is loaded, change the operating directory to two directories back using "cd../.."

3. Type "halt" to shut down the machine, turn off the PC.

\section{Step 6. Powering Down the Etcher}

It is recommended to follow the steps in the order stated.

1. Power off the etcher.

2. Turn off the BetterTech Chiller.

3. Close the $\mathrm{O}_{2}$ gas valve on the cylinder and close the black knob.

4. Close the $\mathrm{N}_{2}, \mathrm{SF}_{6}$, and $\mathrm{C}_{4} \mathrm{~F}_{8}$ gas cylinders located in the blue locker. Ensure to close both the valve and the black knob for each.

5. Move to the gas controller panel located on the wall far right side of the breaker. Close the $\mathrm{N}_{2}$ and $\mathrm{O}_{2}$ knobs by turning them clockwise.

6. Move to the breaker and flip it to the OFF position.

7. Unplug the black cable. 


\section{References}

[1] A. Yariv, Optical Electronics. Saunders College Publishing, 1991.

[2] P. G. J.D. Plummer, M.D. Deal, SILICON VLSI TECHNOLOGY : Fundamentals, Practice and Modeling. Upper Saddle River, New Jersey: Prentice Hall, 2000.

[3] R. J. Shul, G. A. Vawter, C. G. Willison, M. M. Bridges, J. W. Lee, S. J. Pearton, and C. Abernathy, "Comparison of plasma etch techniques for iii-v nitrides," Solid State Electronics, vol. 42, pp. 2259-2267, 1998.

[4] R. G. Hunsperger, Integrated Optics: Theory and Technology. SpringerVerlag, 1982.

[5] G. Reed and A. Knights, Silicon Photonics , An Introduction. John Wiley \& Sons, Ltd, 2004.

[6] R. Soref, J. Schmidtchen, and K. Petermann, "Large single-mode rib waveguides in gesi-si and si-on-so2," J. Quantum Electronics, vol. 27, pp. 19711974, 1991.

[7] S. P. Pogossian, L. Vescan, and A. Vonsovici, "The single-mode condition for semiconductor rib waveguides with large cross section," J. Lightwave Technology, vol. 16, pp. 1851-1853, 1998.

[8] G. T. Reed and C. E. J. Peng, "Silicon optical modulators," materialstoday, vol. 8, pp. 40-50, 2005.

[9] S. Johnson, S. Fan, P. R. Villeneuve, J. Joannopoulos, and L. A. Kolodziejski, "Guided modes in photonic crystal slabs," Phys. Rev. B, vol. 60, pp. 5751$5758,1999$.

[10] B. Jalali, "Can silicon change photonics," Phys. Stat. Sol. (A), vol. 205, pp. 213-224, 2008. 
Sh. Homampour - Masters Thesis

[11] I. Kitay, A. Aydinli, and N. Dagli, "Low-power thermooptical tuning of soi resonator switch," IEEE Photonics Technology Letters, vol. 18, pp. 364-366, 2006.

[12] L. Pavesi, "Will silicon be the photonic material of the third millenium," $J$. Phys.:Condens. Matter, vol. 15, pp. 1169-1196, 2003.

[13] M. Lipson, "Guiding, modulating, and emitting light on silicon-challenges and opportunities," J. Lightwave Technology, vol. 23, pp. 4222-4238, 2005.

[14] A. Liu, R. Jones, L. Liao, D. Samara-Rubio, D. Rubin, O. Cohen, R. Nicolaescu, and M. Paniccia, "A high-speed silicon optical modulation based on a metal-oxide-semiconductor capacitor," Nature, vol. 427, pp. 615-618, Feb. 2004.

[15] A. Othonos and K. Kalli, Fiber Bragg Gratings:Fundamentals and Applications in Telecommunications and Sensing. Boston-London: Artech House, 1999.

[16] K. O. Hill and G. Meltz, "Fiber bragg grating technology fundamentals and overview," J. Lightwave Technology, vol. 15, pp. 1263-1276, 1997.

[17] A. D. Kersey, "A review of recent developments in fiber optic sensor technology," Optical Fiber Technology, vol. 2, pp. 291-317, 1996.

[18] I. J. G. Sparrow, P. G. R. Smith, G. D. Emmerson, S. P. Watts, and C. Riziotis, "Planar bragg grating sensorsfabrication and applications: A review," $J$. Sensors, vol. 2009, 2009.

[19] A. V. Dotsenko, A. L. Diikov, and T. A. Vartanyan, "Label-free biosensor using an optical waveguide with induced bragg grating of variable strength," Sensors and Actuators B, vol. 94, pp. 116-121, 2003.

[20] C. Bruckner, B. Pradarutti, O. Stenzel, R. Steinkopf, S. Riehemann, G. Notni, and A. Tunnermann, "Broadband antireflective surface-relief structure for thz optics," Optixs Express, vol. 15, pp. 779-789, 2007.

[21] H. Nishihara, M. Haruna, and T. Suhara, Optical Integrated Circuits. McGraw-Hill Book Company, 1987.

[22] M. Kamp, J. Hofmann, F. Schafer, M. Reinhard, M. Fischer, T. Bleuel, J. P. Reithmaier, and A. Forchel, "Lateral coupling - a material independent way to complex coupled dfb lasers," Optical Materials, vol. 17, pp. 19-25, 2001. 
[23] F. Ouerghi, W. Belhadj, F. Abdelmalek, M. Mejatty, and H. Bouchriha, "Polymer thin films and bragg grating structures based temperature and pressure integrated effects," Thin Solid Film, vol. 485, pp. 176 - 181, 2005.

[24] D. Runde, S. Brunken, C. E. Ruter, and D. Kip, "Integrated optical electric field sensor based on a bragg grating in lithiumniobate," Applied Physics B, vol. 86, pp. 91-95, 2007.

[25] V. M. N. Passaro, R. Loiacono, G. D’Amico, and F. D. Leonardis, "Design of bragg grating sensors based on submicrometer optical rib waveguides in soi," IEEE Sensors Journal, vol. 8, pp. 1603 - 1611, 2008.

[26] I. J. G. Sparrow, G. D. Emmerson, C. B. E. Gawith, and P. G. R. Smith, "Planar waveguide hygrometer and state sensor demonstrating supercooled water recognition," J. Sensors and Actuators B, vol. 107, pp. 856-860, 2005.

[27] T. Haensel, A. Schindler, and B. Dissing, "Multiple high period accuracy gratings fabricated by holographic exposure and ion beam etching with nanometer depth accuracy in silicon," Optical Manufacturing and Testing $V$, pp. 73-80, 2003.

[28] J. Schrauwen, D. V. Thourhout, and R. Baets, "Focused-ion-beam fabricated vertical fiber couplers on silicon-on-insulator waveguides," Applied Physics Letters, vol. 89, pp. 141102-1 - 141102-3, 2006.

[29] E. C. Baranova, V. M. Gusev, Y. V. Martynenko, C. V. Starinin, and I. B. Haibullin, "On silicon amorphization during different mass ion implantation," Radiation Effects, vol. 18, pp. 21-26, 1973.

[30] C. N. Waddell, W. G. Spitzer, J. E. Fredrickson, G. K. Hubler, and T. A. Kennedy, "Amorphous silicon produced by ion implantation: effects of ion mass and thermal annealing," J. Appl. Phys., vol. 55, pp. 4361-4366, 1984.

[31] H. Y. Fan and A. K. Ramdas, "Infrared absorption and photoconductivity in irradiated silicon," J. Applied Physics, vol. 30, pp. 1127-1134, 1959.

[32] T. Tamir, Guided-Wave Optoelectronics. NewYork: Springer-Verlag, 1990.

[33] E. Hecht, Optics. Addison Wesley, 2002.

[34] R. G. Hunsperger, Integrated Optics: Theory and Techmnology. Springer, 2002. 
[35] J. Lousteau, D. Furniss, A. B. Seddon, T. M. Benson, A. Vukovic, and P. Sewell, "The single-mode condition for silicon-on-insulator optical rib waveguides with large cross section," J. Lightwave Technology, vol. 22, pp. 1923-1929, 2004.

[36] A. G. Rickman, G. T. Reed, and F. Namavar, "Silicon-on-insulator optical rib waveguide loss and mode characteristics," J. Lightwave Technology, vol. 12, pp. 1771-1776, 1994.

[37] D. L. Lee, Electromagnetic Principles of Integrated Optics. Wiley, 1986.

[38] R. Scarmozzino, "Numerical techniques for modeling guided-wave photonic devices," IEEE J. Selected Topics in Quantum Electronics, vol. 6, pp. 150$162,2000$.

[39] D. L. Jaggard and C. Elachi, "Floquet and coupled-waves analysis of higherorder bragg coupling in a periodic medium," J. Opt. Soc. Am., vol. 66, pp. 674-682, 1976.

[40] B. E. Little, "A variational coupled-mode theory including radiation loss for grating-assisted couplers," J. Lightwave Technology, vol. 14, pp. 188-195, 1996.

[41] T. K. Gaylord and M. G. Moharam, "Analysis and applications of optical diffraction by gratings," IEEE Proc., vol. 73, pp. 894-938, 1985.

[42] S. Park, S. H. Song, C. Oh, and P. Kim, "Ray-optical determination of the coupling coefficients of grating waveguide by use of the rigorous coupledwave theory," J. Lightwave Technology, vol. 19, pp. 120-125, 2001.

[43] P. Verly, R. Tremblay, and J. W. Y. Lit, "Application of the effective-index method to the study of distributed feedback in corrugated waveguides. te polarization," J. Opt. Soc. Am., vol. 70, pp. 964-968, 1980.

[44] G. Lifante, Integrated Photonics Fundamentals. Wiley, 2003.

[45] L. Zeng, Y. Yi, C. Hong, J. Liu, N. Feng, X. Duan, L. C. Kimerling, and B. A. Alamariu, "Efficiency enhancement in si soalr cells by textured photonic crystal back reflector," Appl. Phys. Lett., vol. 89, p. 111111, 2006.

[46] L. Zeng, P. Bermei, Y. Yi, B. A. Alamariu, K. A. Broderick, J. Liu, C. Hong, X. Duan, J. Joannopoulos, and L. C. Kimerling, "Demonstration of enhanced absorption in thin film si solar cells with textured photonic crystal back reflector," Appl. Phys. Lett., vol. 93, p. 221105, 2008. 
Sh. Homampour - Masters Thesis

[47] T. Tiedje, B. Abeles, J. M. Cebulka, and J. Pelz, "Photoconductivity enhancment by light trapping in rough amorphous silicon," Appl. Phys. Lett., vol. 42, pp. $712-714,1983$.

[48] D. C. Johnson, I. Ballard, K. W. J. Barnham, D. B. Bishnell, J. P. Connolly, M. C. Lynch, T. N. D. Tibbits, N. J. Ekins-Daukes, M. Mazzer, R. Airey, G. Hill, and J. Roberts, "Advances in bragg stack quantum well solar cells," Sol. Energy mater. Sol. Cells, vol. 87, pp. 169 - 179, 2005.

[49] D. Taillaert, W. Bogaerts, P. Bienstman, T. F. Krauss, P. V. Daele, I. Moerman, S. Verstuyft, K. D. Mesel, and R. Baets, "An out-of-plane grating coupler for efficient butt-coupling between compact planar waveguides and single-mode fibers," IEEE J. Quantum Electronics, vol. 38, pp. 949-955, 2002.

[50] R. Orobtchouk, A. Layadi, H. Gualous, D. Pascal, A. Koster, and S. Laval, "High-efficiency light coupling in a submicrometric silicon-on-insulator waveguides," Appl. Opt., vol. 39, pp. 5773-5777, 2000.

[51] D. Taillaert, P. Bienstman, and R. Baets, "Compact effecient broadband grating coupler for silicon-on-insulator waveguides," Optics Lett., vol. 29, pp. 2749-2751, 2004.

[52] P. J. Foster, J. K. Doylend, P. Mascher, and A. P. Knights, "Optical attenuation in defect-engineered silicon rib waveguides," J. Appl. Phys., vol. 99, p. 073101, 2006.

[53] M. J. A. de Dood, A. Polman, T. Zijlstra, and E. W. J. M. van der Drift, "Amorphous silicon waveguides for microphotonics," J. Appl. Phys., vol. 92, pp. 649-653, 2002.

[54] L. Pelaz, L. A. Marqus, and J. Barbolla, "Ion-beam-induced amorphization and recrystallization in silicon,” J. Appl. Phys., vol. 96, pp. 5947-5976, 2004.

[55] J. W. Corbett, Electron Radiation Damage In Semiconductors and Metals, Supplement \&. Academic Press, 1966.

[56] G. K. Hubler, C. N. Waddell, W. G. Spitzer, J. E. Fredrickson, S. Prussin, and R. G. Wilson, "High-fluence implantations of silicon: layer thickness and refractive indices," J. Appl. Phys., vol. 50, pp. 3294-3303, 1979.

[57] F. F. Morehead, B. L. Crowder, and R. S. Title, "Formation of amorphous silicon by ion bombardment as a function of ion, temperature, and dose," $J$. Appl. Phys., vol. 43, pp. 1112-1118, 1972. 
Sh. Homampour - Masters Thesis

Engineering Physics, McMaster University

REFERENCES

[58] J. M. Haile, Molecular Dynamics Simulation : Elementary Methods. Wiley, 1992.

[59] I. Manning, "Transport-theory approach to ion-beam mixing and recoil implantation," J. Phys. Rev. B, vol. 42, pp. 9853-9858, 1990.

[60] M. T. Robinson, "Binary collision approximation : Background and introduction," Radiat. Eff., vol. 130-131, pp. 3-20, 1994.

[61] E. Rimini, Ion-Implantation : Basics to Device Fabrication. Kluwer Academic, 1994.

[62] M. Bulk, "Ion implanted bragg gratings in silicon-on-insulator rib waveguides," Master's thesis, Engineering Physics, McMaster University, 2008.

[63] W. Wesch, E. Glaser, G. Gotz, H. Karge, and R. Prager, "Correlation between structural defects and optical properties in ion-implanted silicon," Phys. Stat. Sol. a, vol. 65, pp. 225-232, 1981.

[64] J. S. Custer, M. O. Thompson, D. C. Jacobson, J. M. Poate, S. Roorda, W. C. Sinke, and F. Spaepen, "Density of amorphous si," J. Appl. Phys. Lett., vol. 64, pp. 437-439, 1994.

[65] U. Zammit, K. N. Madhusoodanan, M. Marinelli, F. Scudieri, R. Pizzoferrato, F. Mercuri, and E. Wendler, "Optical absorption studies of ion implanted and amorphous silicon," J. De Physique IV, vol. 4, pp. C7113 C7120, 1994.

[66] S. Roorda, W. C. Sinke, J. M. Poate, D. C. Jacobson, S. Dierker, B. S. Dennis, D. J. Eaglesham, F. Spaepen, and P. Fuoss, "Structural relaxation and defect annihilation in pure amorphous silicon," J. Phys. Rev. B, vol. 44, pp. $3702-$ $3725,1991$.

[67] J. E. Fredrickson, C. N. Waddell, W. G. Spitzer, and G. K. Hubler, "Effects of thermal annealing on the refractive index of amorphous silicon produced by ion implantation," Chemical Technology Review, pp. 219-229, 1983.

[68] A. Mathew and K. N. Madhusoodanan, "Subgap optical absorption studies in boron implanted silicon," Japanese Journal of Applied Physics, vol. 43, pp. 5088-5094, 2004.

[69] H. J. Stein, F. L. Vook, D. K. Brice, J. A. Borders, and S. T. Picraux, "Infrared studies of the crystallinity of ion-implanted si," Radiat. Eff., vol. 6, pp. 1926, 1970. 
Sh. Homampour - Masters Thesis

[70] L. J. Cheng, J. C. Corelli, J. W. Corbett, and G. D. Watkins, "1.8-, 3.3-, and 3.9- mu bands in irradiated silicon: Correlations with the divacancy," Phys. Rev., vol. 152, pp. 761-774, 1966.

[71] P. G. Coleman, C. P. Burrows, and A. P. Knights, "Simple expression for vacancy concentrations at half ion range following mev ion implantation of silicon," J. Appl. Phys. Lett., vol. 80, pp. 947-949, 2002.

[72] K. David, J. Buus, G. Morthier, and R. Baets IEEE Photonics Technology Letters, vol. 3, pp. 439-441, 1991.

[73] P. Brosson, C. Artigue, B. Fernier, D. Leclerc, J. Jacquet, and J. Benoit, "Simple determination of coupling coefficient in dfb waveguide structures," Electron. Lett., vol. 24, pp. 990-991, 1988.

[74] A. Yariv, P. Yeh, and A. Yariv, Photonics : Optical Electronics in Modern Communications. Oxford University Press, 2007.

[75] H. M. H. Chong and R. M. D. L. Rue, "Tuning of photonic crystal waveguide microcavity by thermooptic effect," IEEE Photonics Techenology Lett., vol. 16, pp. 1528-1530, 2004.

[76] F. Gan, T. Barwicz, M. A. Poppvic, M. S. Dahlem, C. W. Holzwarth, P. T. Rakich, H. I. Smith, E. P. Ippen, and F. X. Kartner, "Maximizing the thermooptic tuning range of silicon photonic structures," IEEE Photonics in Switching, pp. 67-68, 2007.

[77] L. Domash, M. Wu, N. Nemchuk, and E. Ma, "Tunable and switchable multiple-cavity thin film filters," J. Lightwave Technology, vol. 22, pp. 126135, 2004.

[78] I. Giuntoni, A. Gajda, M. Krause, R. Steingruber, J. Bruns, and K. Petermann, "Tunable bragg reflector on silicon-on-insulator rib waveguides," $\mathrm{Op}$ tics Express, vol. 17, pp. 18518-18524, 2009.

[79] B. Wild, R. Ferrini, R. Houdre, M. Mulot, S. Anand, and C. J. M. Smith, "Temperature tuning of the optical properties of planar photonic crystal microcavities," J. Appl. Phys. Lett., vol. 84, pp. 846-848, 2004.

[80] W. C. L. Hopman, P. Pottier, D. Yudistira, J. van Lith, P. V. Lambeck, R. M. D. L. Rue, A. Driessen, H. J. W. M. Hoekstra, and R. M. de Ridder, "Quasione-dimensional photonic crystal as a compact building-block for refractometric optical sensors,' IEEE J. Selected Topics in Quantum Electronics, vol. 11, pp. 11-16, 2005. 
Sh. Homampour - Masters Thesis

Engineering Physics, McMaster University

REFERENCES

[81] R. L. Espinola, M. C. Tsai, J. T. Yardley, and R. M. Osgood, "Fast and lowpower thermooptic switch on thin silicon-on-insulator," Photonics Technology Lett., vol. 15, pp. 1366-1368, 20003.

[82] R. Brandt and G. Neuer, "Electrical resistivity and thetmal conductivity of pure aluminum and aluminum alloys up to and above the melting temperature," International Journal of Thermophysics, vol. 28, pp. 1429-1446, 2007.

[83] F. Magno, F. D. Olio, and V. M. N. Passaro, "Multiphysics investigation of thermo-optic effect in silicon-on-insulator waveguide arrays," Proceedings of the COMSOL Users Conference, 2006.

[84] G. K. Celler and S. Cristoloveanu, "Frontiers of silicon-on-insulator," J. Appl. Phys., vol. 93, pp. 4955-4978, 2003.

[85] J. Schmidtchen, A. Splett, B. Schueppert, K. Petermann, and G. Burbach, "Low loss single mode optical waveguides with large cross-section in siliconon-insulator," Electron. Lett., vol. 27, pp. 1486-1488, 1991.

[86] T. Wells, M. M. El-Gomati, and J. Wood, "Low temperature reactive ion etching of silicon with sf6 / o2 plasmas," J. Vacuum Science and Technology $B$, vol. 15, pp. 434-438, 1997.

[87] R. A. Ghanbari, Physics and Fabrication of Quasi-One-Dimensional Conductors. PhD thesis, MIT, 1993.

[88] K. O. Hill, B. Malo, F. Bilodeau, D. C. Johnson, and J. Albert, "Bragg gratings fabricated in monomode photosensitive optical fiber by uv exposure through a phase mask," J Appl. Phys. Lett., vol. 62, pp. 1035-1037, 1993.

[89] D. J. Moss, V. G. Taeed, B. J. Eggleton, D. Freeman, S. Madden, M. Samoc, B. Luther-Davies, S. Janz, and D.-X. Xu, "Bragg gratings in silicon-oninsulator waveguides by focused ion beam milling," J. Appl. Phys. Lett., vol. 85, pp. 4860-4862, 2004.

[90] J. Schrauwen, F. V. Laere, D. V. Thourhout, and R. Baets, "Focused-ionbeam fabrication of slanted fiber couplers in silicon-on-insulator waveguides," IEEE J. Photonics Technology Lett., vol. 19, pp. 816-818, 2007.

[91] L. Guo, P. R. Krauss, and S. Y. Choub, "Nanoscale silicon field effect transistors fabricated using imprint lithography," J. Appl. Phys. Lett., vol. 71, pp. 1881-1883, 1997.

[92] J. G. Goodberlet, "Patterning $100 \mathrm{~nm}$ features using deep-ultraviolet contact photolithography," J. Appl. Phys. Lett., vol. 76, pp. 667-669, 2000. 
Sh. Homampour - Masters Thesis

[93] R. T. S. Wolf, Silicon Processing for the VLSI Era, Volume 1-Process Technology, vol. 1. P.O. BOX 340, Sunset Beach, California: Lattice Press, 1986.

[94] M. Madou, Fundamentals of MICROFABRICATION :The science of miniaturization. CRC Press, 2002.

[95] R. Shul, G. McClellan, S. Casalnuovo, D. Rieger, S. Pearton, C. Constantine, C. Barratt, R. Karlicek, C. Tran, and M. Schurman, "Inductively coupled plasma etching of gan," Applied Physics Letter, vol. 69, no. 8, pp. 11191121, 1996.

[96] K. S. Chen, A. A. Ayon, X. Zhang, and S. M. Spearing, "Effect of process parameters on the surface morphology and mechanical performance of silicon structure after deep reactive ion etching (drie)," J. Microelectromehcanical Sysytems, vol. 11, pp. 264-275, 2002.

[97] N. Roxhed, P. Griss, and G. Stemme, "A method for tapered deep reactive ion etching using a modified bosch process," J. Micromech. Microeng, vol. 17, pp. 1087-1092, 2007.

[98] M. A. Blauw, G. Craciun, W. G. Sloof, P. J. French, and E. van der Drift, "Advanced time-multiplexed plasma etching of high aspect ratio silicon structures," J. Vacuum Science Technology, B, vol. 20, pp. 3106-3110, 2002.

[99] R. Knizikevicius and V. Kopustinskas, "Anisotropic etching of silicon in sf6 plasma," Vacuum, vol. 77, pp. 1-4, 2004.

[100] D. Mannos and D. Flamm, Plasma Etching. Academic Press, 1989.

[101] M. Pichot, "Dry processing in microelectronics: toward low pressure plasma technology," Vacuum, vol. 41, pp. 895-898, 1990.

[102] D. C. Gray, I. Tepermeister, and H. H. Sawin, "Phenomenological modeling of ion-enhanced surface kinetics in fluorine-based plasma etching," J. Vacuum Science Technology B, vol. 11, pp. 1243-1257, 1993.

[103] S. K. Pani, C. C. Wong, K. Sudharsanam, S. G. Mhaisalkar, V. Lim, S. Mohanraj, and P. V. Ramana, "Effect of process parameters on sidewall roughness in polymeric optical waveguides," Thin Solid Films, vol. 462-463, pp. 471-476, 2004.

[104] R. Doering, Handbook of Semiconductor Manufacturing Technology. CRC Press, 2008. 
Sh. Homampour - Masters Thesis

Engineering Physics, McMaster University

REFERENCES

[105] R. F. Figueroa, S. L. Burkett, and L. Schaper, "Control of sidewall slope in silicon vias using sf6 / o2 plasma etching in a conventional reactive ion etching tool," J. Vacuum Science and Technology B, vol. 23, pp. 2226-2231, 2005.

[106] J. P. P. P. N. Agarwal, S. ponoth, “Optimized oxygen plasma etching of polyimide films for low loss optical waveguides," J. Vacuum Science and Technology A, vol. 20, pp. 1587-1591, 2002.

[107] J. V. Aelst, H. Struyf, W. Boullart, and S. Vanhaelemeersch, "High aspect ratio via etch development for cu nails in 3-d- stacked ics," Thin Solid Films, vol. 516, pp. 3502-3506, 2008.

[108] W. H. Juan and S. W. Pang, "Controlling sidewall smoothness for micromachined si mirrors and lenses," J. Vac. Sci. Technol. B, vol. 16, pp. 4080-4084, 1996.

[109] T. E. Murphy, H. J. T, and H. I. Smith, "Fabrication and characterization of narrow-band bragg-reflection filters in silicon-on-insulator ridge waveguides," J. Lightwave Technology, vol. 19, pp. 1938-1942, 2001.

[110] R. B. Roberts, "Thermal expansion reference data: silicon 300-850 k," J. Phys. D : Appl. Phys., vol. 14, pp. L163-6, 1981. 